Copyright by

\title{
CAROL LARSON
}




\title{
A Cognitive Prototype Model of Moral Judgment and Disagreement
}

\author{
BY \\ CAROL LARSON \\ B.S., Southern Illinois University, Carbondale, IL 1990 \\ M.S., New York University, New York City, NY 1995 \\ C.A.S., University of Illinois, Champaign-Urbana, IL 2002
}

\section{THESIS}

Submitted as partial fulfillment of the requirements

for the degree of Doctor of Philosophy in Educational Psychology

in the Graduate College of the

University of Illinois at Chicago, 2012

Chicago, Illinois

Defense Committee:

Stacey Horn, Chair

Larry Nucci, Advisor, Professor Emeritus

Everett Smith

Kim Lawless

Cecilia Wainryb, University of Utah 
To my husband. I could not have done this without you.

To my daughters. You motivated and inspired me to do my best.

To my father. You taught me the value of hard-work, responsibility, and resilience, which gave me the will to finish this thesis.

And to my mother. From Day 1, you promised me an education, even if it meant you having to work 3 jobs.

This project is for you, mom. 


\section{ACKNOWLEDGMENTS}

I would like to thank my dissertation committee: Larry Nucci, Stacey Horn, Everett Smith, Cecilia Wainryb, and Kim Lawless. They challenged me to go beyond the expectations that I set for myself. Their collective commitment, experience, and expertise enriched this dissertation and provided me with one of my most valuable experiences.

I would also like to thank the University of Illinois at Chicago for the Provost's Award and the State of Illinois for the Diversifying Faculty of Illinois Fellowship, which helped to support my research.

CL 


\section{TABLE OF CONTENTS}

\section{CHAPTER}

PAGE

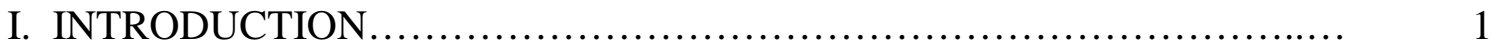

II. CONCDEPTUAL FRAMEWORK AND LITERATURE REVIEW ........... 7

A. Contemporary Formulations of Moral Disagreement................. 7

1. MacIntyre: Incommensurable Moral Goods...................... 7

2. Shweder: The "Big Three" of Morality and Diverging Moral Codes 12

3. Turiel: Socio-cognitive Domain Theory..................... 16

4. Haidt: Social Intuitionist Model of Moral Judgment............ 20

5. Churchland: the Moral Network Theory...................... 25

6. What Can We Learn From these Scholars?..................... 30

B. The Properties of Moral Prototypes............................. 31

1. Social Context............................................. 31

2. Autonomy and Consent..................................... 36

3. Intentions and Outcomes.................................... 42

C. Moral Judgment and Disagreement: A Cognitive Prototype Perspective 48

D. Testing the Model.................................................. 63

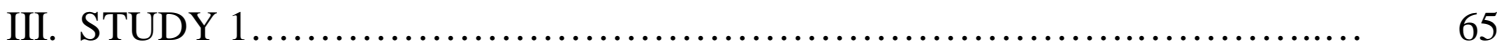

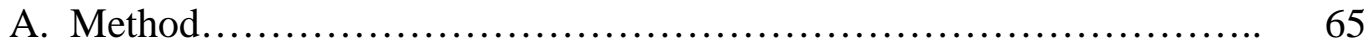

1. Purpose................................................... 65

2. Participants............................................ 66

3. Measures and Procedures.................................... 67

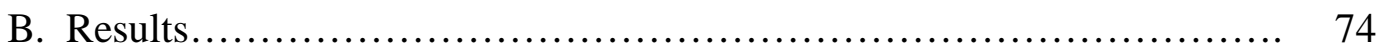

1. Data Analysis Plan and Data Reduction........................ 74

2. Moral Judgment Ratings........................................ 79

3. Moral Judgment Frequencies and Percentages................... 83

4. Moral Justifications........................................ 85

C. Discussion................................................... 100

1. Moral Judgment Ratings.................................... 100

2. Moral Judgment Frequencies and Percentages..................... 105

3. Moral Justifications.......................................... 106

4. Limitations and Suggestions for Future Research................ 110

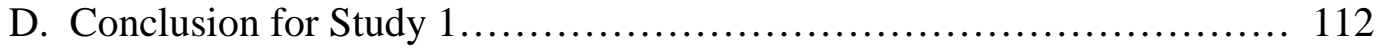

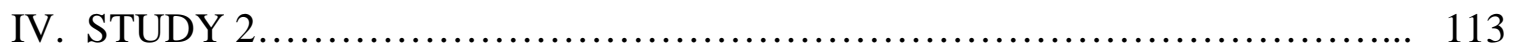

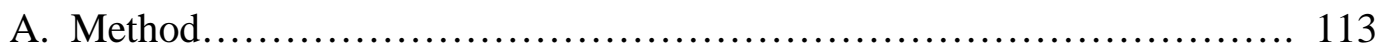

1. Purpose.................................................... 113

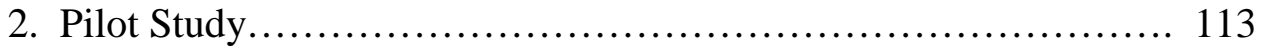

3. Participants............................................... 117

4. Measures and Procedures....................................... 119 


\section{TABLE OF CONTENTS (continued)}

CHAPTER

PAGE

IV. STUDY 2 (continued)

B. Results.

1. Data Analysis Plan and Data Reductions...................... 124

2. Moral Judgment Ratings................................... 132

3. Moral Judgment Frequencies and Percentages................ 138

4. Moral Justifications...................................... 140

C. Discussion.................................................. 158

1. Moral Judgment Ratings................................... 158

2. Moral Judgment Frequencies and Percentages.................. 169

3. Moral Justifications...................................... 172

4. Limitations and Suggestions for Future Research............. 175

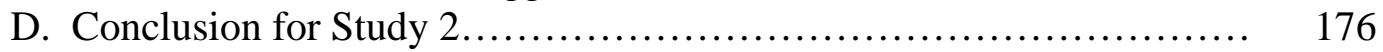

V. GENERAL DISCUSSION AND IMPLICATIONS......................... 178

A. General Discussion............................................ 178

B. Implications................................................ 190

1. Revision of the Blame Intensification Hypothesis............... 190

2. A Moral Judgment Calculus................................. 191

3. Connections to Moral Development and Education............. 192

4. Recommendation for Policymakers........................ 193

REFERENCES.................................................... 195

ENDNOTES........................................................ 209

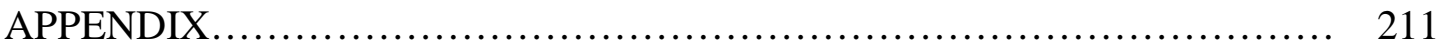

A. Study 1 Survey Questions...................................... 211

B. Study 2 Survey Questions....................................... 215

C. IRB Approval Letter....................................... 220

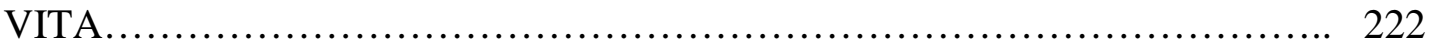




\section{LIST OF TABLES}

TABLE

PAGE

I. DEFINITIONS AND SAMPLES OF JUSTIFICATION CATEGORIES ..... 68

II. DEFINITIONS AND SAMPLES OF JUSTIFICATION CATEGORIES FOR THE NO PROPERTIES CASES ................................ 70

III. SUBCATEGORIES OF JUSTIFICATIONS CODED AS "NO PROPERTY" ACTUAL NUMBER (\% OF TREATMENT GROUP) [\% OF TOTAL] PER SCENARIO......................................................... 78

IV. MORAL JUDGMENT RATINGS BY CONDITION AND SCENARIO

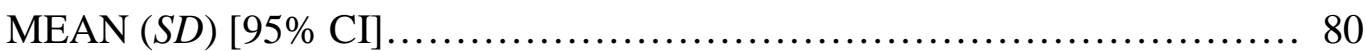

V. FREQUENCY (AND PERCENTAGE) OF WRONG AND RIGHT RESPONSES 84

VI. PAIRWISE COMPARISONS (BONFERRONI) OF MORAL JUSTIFICATIONS IN THE HITTING SCENARIO. 86

VII. PROPORTIONAL MEANS (SD) [95\% CI] BY CELL FOR EACH SCENARIO. 88

VIII. PAIRWISE COMPARISONS (BONFERRONI) OF MORAL JUSTIFICATIONS IN THE PROPERTY DESTRUCTION SCENARIO.... 91

IX. PAIRWISE COMPARISONS (BONFERRONI) OF MORAL JUSTIFICATIONS IN THE STEALING SCENARIO

X. PAIRWISE COMPARISONS (BONFERRONI) OF MORAL JUSTIFICATIONS IN THE KILLING SCENARIO.

XI. RESULTS OF SOCIAL CONTEXT VARIABLE AGREEMENT.......... 115

XII. RESULTS OF VARIABLE MANIPULATION CHECK.................. 117

XIII. DEFINITIONS AND SAMPLES OF JUSTIFICATION CATEGORIES.... 122

XIV. PEARSON CORRELATIONS OF DEPENDENT VARIABLES........... 125

XV. PEARSON CORRELATIONS OF SCENARIOS’ LEVELS OF RIGHT/WRONG WITHIN EACH TREATMENT CONDITION 
LIST OF TABLES (continued)

TABLE

PAGE

XVI. SUBCATEGORIES OF JUSTIFCATIONS CODED AS

"NO PROPERTY" ACTUAL NUMBER (\% OF TREATMENT GROUP)

[\% OF TOTAL] PER SCENARIO.

130

XVII. MEAN (SD) [95\% CI] LEVELS OF RIGHT/WRONG, PRAISE/BLAME AND DIFFICULTY BY CONDITION

XVIII. FREQUENCY AND (PERCENTAGE) OF WRONG AND RIGHT

RESPONSES

XIX. PAIRWISE COMPARISONS (BONFERRONI) OF MORAL JUSTIFICATIONS IN THE CLONING SCENARIO.

XX. PROPORTIONAL MEANS (SD) [95\% CI] BY CELL FOR EACH SCENARIO.

XXI. PAIRWISE COMPARISONS (BONFERRONI) OF MORAL JUSTIFICATIONS IN THE MARRIAGE SCENARIO.

XXII. PAIRWISE COMPARISONS (BONFERRONI) OF MORAL JUSTIFICATIONS IN THE FACTORY SCENARIO

XXIII. PAIRWISE COMPARISONS (BONFERRONI) OF MORAL JUSTIFICATIONS IN THE ORPHANAGE SCENARIO.

XXIV. STRUCTURE OF IMMORAL CLONING SCENARIO................... 160

XXV. STRUCTURE OF IMMORAL MARRIAGE SCENARIO................. 161

XXVI. STRUCTURE OF PROMORAL FACTORY SCENARIO................ 162

XXVII. STRUCTURE OF PROMORAL ORPHANAGE SCENARIO............. 163 


\section{LIST OF FIGURES}

FIGURE

PAGE

1. Property orientations of immoral and promoral prototypes......... 51

2. Graphic display of a nonprototype example.................... 57

3. Structure of ANOVA research design by groups................. 75

4. Line graph of each scenario’s moral judgment means by condition... 103

5. Structure of the MANOVA research design by groups............. 127 


\section{SUMMARY}

According to the cognitive prototype model of moral judgment and disagreement, moral prototypes consist of the following properties: social context, intentions, consent/autonomy, and outcomes. Over time and as a result of developmental interactions in the social environment, the properties are abstracted from experiences and used to construct immoral and promoral prototypes. Promoral prototypes represent actions that are encouraged and considered virtuous by the social group, intentionally benevolent and other-regarding, consensual, and resulting in positive outcomes. Conversely, immoral prototypes represent actions that are discouraged and not condoned by the social group, intentionally malevolent or self-serving, performed against the will of the target, and resulting in bad outcomes. Nonprototypes are situations that deviate from the prototypes and may not be as easy to categorize. This model posits that moral prototypes will result in a high level of agreement while nonprototypes will often act as a source of moral disagreement.

Using this framework, two studies were performed. Study 1 presented 233 adult participants with abstract moral transgressions and then contextualized them by gradually adding explicit layers of promoral property language. Contextualized immoral prototypes with explicitly stated immoral properties were also represented in the scenario sets. The participants were asked to rate each scenario and provide a written explanation. The findings in this study suggest that the abstract transgressions and contextualized prototypes generate similar judgments and result in perfect or near perfect agreement that the situation is wrong. Greater disagreement appears to become manifest when the 


\section{SUMMARY (continued)}

participants judge nonprototypes. In general, the abstract transgressions and contextualized prototypes were often rated as more wrong than the nonprototypes and never judged to be more right. Additionally, this study found that the promoral intentions and consent properties appear to carry weight and may produce additive affects when they are combined. The shifting judgments in this study support the hypothesis that inferences are made when the properties are not made explicit. Although the justifications provided additional evidence that the participants inferred the orientation of an inexplicit property or relied on a default immoral prototype when presented with an abstract transgression, more interview-centered research is needed to further test this hypothesis. Finally, the findings in this study suggest that justification language will vary according to how a moral situation is presented.

In the second study, 481 adult participants read scenarios that represented moral prototypes and nonprototypes. They were asked to rate the scenarios according to levels of right/wrong, praise/blame, and difficulty. The promoral prototype conditions resulted in significantly higher levels of rightness and praise than most or all of the nonprototypes, and the immoral prototypes received higher levels of wrongfulness and blame than most or all of the nonprototypes in each respective scenario set. None of the nonprototype conditions received significantly higher levels of right/wrong or praise/blame than their respective prototype conditions in each scenario set. The difficulty level did not yield consistent patterns across the four scenario sets. However, none of the nonprototypes 


\section{SUMMARY (continued)}

were easier to judge than the prototypes. When the participants explained their responses, the type of condition and scenario seemed to have a bearing their justifications. Immoral scenarios produced a predominant justification, while the pormoral scenario justifications were more sensitive to the type of conflict presented in a given condition. Prototypes, across all four scenario sets, resulted in near perfect or perfect agreement. Some nonprototype conflict conditions in the immoral and promoral scenario sets resulted in disagreement, although there was a greater range of disagreement among the nonprototypes in the promoral scenarios. Finally, although no hypothesis testing was conducted to compare promoral and immoral conditions, this study appears to contribute further evidence that people judge promoral and immoral situations differently. 


\section{CHAPTER I: INTRODUCTION}

By the spring of 2005, nearly every newspaper, television news program, and talk radio show had reported on the unusual family and federal legal battle concerning the Schiavo right-to-die/right-to-live case. Debates about Terri Schiavo’s plight were heated, ubiquitous, and intensely political as they pricked the nerve of America's moral consciousness. According to newspaper reports (Goodnough, Carey, Jordan Sexton, \& Yardley, 2005; Kampert \& Martinez, 2003), Terri, who was living in St. Petersburg, Florida at the time, suffered a cardiac arrest, which left her brain damaged and in a persistent vegetative state that rendered her incapable of thought or emotion. From 1998 - 2005, Terri’s parents and her husband, Michael Schiavo, fought over the guardianship rights that would ultimately determine Terri's fate. Michael Schiavo claimed that his wife had said on several occasions that she did not want to be dependent on lifesupporting machines, which was the basis for his decision to remove the feeding tube that kept Terri alive. Terri's parents, however, continued to believe that there was hope and tenaciously challenged Michael Schiavo in a series of legal battles. In the end, the courts sided with Michael Schiavo and the feeding tube was removed. Terri died on March 31, 2005.

Throughout this ordeal, Americans grappled with many questions and points of view. Some believed that Terri had the right to die if that is what she wanted and to not honor that would condemn her to live a life of prolonged suffering (McNelis, 2003). In a New York Times editorial entitled, “Terri Schiavo and the Moral Divide” an anonymous 
physician writes, “The primary consideration when determining whether someone's life should or should not be artificially prolonged in a vegetative state should be the individual's expressed wishes” ("Terri Schiavo," 2005, p.16).

On the other side of the moral divide were people (particularly conservative and religious groups) who felt that Terri had the right to live and believed that we had a moral obligation to keep her alive. James Q. Wilson (2005), Public Policy Scholar and author of The Moral Sense, argued that the feeding tube should not have been withdrawn from Terri Schiavo, because the whole family did not consent to removing the tube. In addition, he claimed that there were doctors who believed that Terri's condition could improve, even though the chances of this happening were extremely small. Given that there was a smidgen of hope for some recovery, Wilson maintained that removing the tube could be construed as murder, depending on the circumstances. Finally, as did many others who were familiar with the case, Wilson speculated what Michael Schiavo's intentions were as reflected in the following statement:

[Terri's] parents have begged to become her guardians. Her husband has refused. We do not know for certain why the husband has refused. I doubt that he wished to receive for himself the money that still exists from her insurance settlement and, apparently, he has offered to donate that money to charity. Perhaps, being a Catholic, he would like her death to make him free to marry the woman with whom he is now living. Or perhaps (and I think that this is most likely the case) he does not want to live what strikes him as an intolerable life. (p. A16) Jeb Bush, the governor of Florida who exercised his political muscle for the purpose of saving Terri's life, claimed that this case was the toughest issue of his career 
(Goodnough et al., 2005). Not only was this case rife with conflicts pertaining to the intentions of the key players, consent issues, and outcomes that pitted prolonging life against ending suffering; but also within the social context, people argued over conceptual meanings. Some people asked, what does it mean to be in a persistent vegetative state? Is it murder to remove a feeding tube from a person in a persistent vegetative state? What does it mean to live a quality life? Among those who grappled with these questions, Wilson (2005) argues:

The intolerable life argument has support from many doctors and bioethicists. They claim that a person can be "socially dead" even when their brains can engage in some functions. By "socially dead" they mean that the patient is no longer a person in some sense. At this point their argument gets a bit fuzzy because they must somehow define what is a "person" and a "non-person." That is no easy matter. (p. A16)

The Terri Schiavo case is just one scenario among many that has reverberated with moral discord at a national level. Should Elian Gonzalez stay in the U.S. or return to Cuba? Should we engage in stem cell research? Is abortion murder? These are all questions that have led to great debates and conflicts among people living in the same social milieu. And yet, there are other moral situations in which there is little contention or refutation.

\section{Research Problem}

It is believed that morality is a universal human phenomenon (Kohlberg, Levine, \& Hewer, 1983). Some have speculated that the development of innate processes and our interaction with the social environment have contributed to the evolution of a moral 
system that enables us to strengthen social relationships, identify and contend with the “cheaters” in a given society, and ultimately, improve our chances for survival via cooperation (Kohlberg et al., 1983; Krebs \& Denton, 1997; Krebs et al., 2002; Pinker, 2002; Waal, 1996; Wilson, 1993). Although humans possess the ability to construct moral knowledge, we do not always form the same moral conclusions, as depicted by the Terri Schiavo case. Additionally, not every moral issue requires the same magnitude of cognitive processing. Some moral judgments feel automatic and effortless while others may require us to grapple for extended periods of time. Understanding the inconsistencies in moral judgment is necessary, because diverging conclusions about highly charged, moral situations often lead to conflict, and in some cases, may incite acts of violence (e.g., the bombing of abortion clinics). As such, it is important to learn how psychological processes drive moral judgments and how moral disagreement becomes manifest.

The proposed research that follows will identify the shortcomings and significant contributions of prior theories and use this analysis to construct a new perspective that will explain the phenomenon of moral disagreement via a cognitive prototype model. Although this is not the first model to use prototypes in a theory of morality, Churchland's (1998) moral network theory does not identify the properties of moral issues. In order to account for this problem, this cognitive prototype perspective identifies four key attributes of moral reasoning from a review of empirical research relevant to making moral judgments. The four key attributes are as follows:

Social context. The transmission and construction of knowledge and values are influenced by a social context. In other words, what is perceived to be true, good, 
beautiful, desirable, and virtuous influences how we interpret moral situations via a socially meaningful lens. In addition to research in cultural psychology (e.g., Shweder, Mahapatra, \& Miller, 1987), empirical studies on how informational assumptions affect moral reasoning support this idea (Turiel, Hildebrandt, \& Wainryb, 1991; Wainryb, 1993). For example, Smetana (1981) found that women's beliefs about abortion are influenced by perceptions of when life begins. These values and beliefs lead social groups to condone or condemn particular practices, actions, or events. Thus, the social context bears on how moral situations are interpreted, which leads to moral prescriptions of actions that are considered good or bad within the cultural milieu.

Intentions. Intentions and intentionality have to do with whether or not an agent engages in purposeful action to achieve a targeted end. It has been found that one's intentions are a salient dimension in the formation of moral judgments (Jones \& Nelson-Le Gall, 1995; Kordes-de Vaal, 1996; Smetana, Campione-Barr, \& Yell, 2003; Zelazo, Helwig, \& Lau, 1996).

Consent. Morality is a social phenomenon that is other-regarding. In other words, moral situations require us to think about how an individual’s (or group’s) actions directly or indirectly affect others. Thus, consent issues (i.e., issues having to do with whether or not there is justified or unjustified acquiescence, force, coercion, or deception) is another important feature relevant to moral reasoning (see Nucci \& Smetana, 1996 and Smetana, 2005 for relevant research on the personal domain). 
Outcomes. When making moral judgments, people often take into account what the outcome of the action is. Research in moral development has found that both children and adults consider outcomes in formulating moral judgments (Baron, 2002; Jones \& Renick Thomson, 2001; Piaget, 1932/1965; Zelazo et al., 1996). In short, the main goal of this research is to explore the following questions: 1.$)$ How does moral disagreement become manifest? 2.) In what ways do these proposed properties affect moral judgment? 3.) Is moral agreement possible? If so, under what conditions? The cognitive prototype perspective will argue that the attributes listed above are properties of moral prototypes and have a collective effect on the moral judgments we make. In other words, these properties, which are abstracted as we construct moral knowledge and accumulate moral experience, become salient within the context of making judgments and have a bearing on how we reason about issues in the moral domain. The relationships of these properties to each other lead to the construction of categories that delineate between what is considered morally "good" or praiseworthy and what is morally "bad" or blameworthy. Within this view, moral situations that do not neatly fit into these categories (i.e., situations deviate from the prototype) are more prone to moral disagreement. 


\section{CHAPTER II: CONCEPTUAL FRAMEWORK AND LITERATURE REVIEW Contemporary Formulations of Moral Disagreement}

For centuries there have been great debates about the nature of morality and the sources of moral disagreement. At one end of the spectrum, it is believed that morality is a rational event, and as a result of pure reasoning, one can discover universal moral truths that will ultimately resolve moral disagreements (Kant, 1785/1998; Kohlberg et al., 1983). Supporters of this point of view believe that an objective, universal moral code exists. However, at the other end of the spectrum, perpetuating, interminable moral disagreements have fueled a position that there are no universal moral truths (i.e., all people do not share the same moral code). Supporters of this position believe that local conditions, cultural worldviews, and one's passions shape our moral sensibilities (Haidt, 2001; Posner, 1999).

There are several important theories that identify, either explicitly or implicitly, sources of moral disagreement. Each of these theories attempts to explain why certain polemics (such as abortion, female genital circumcision, euthanasia, etc.) create great chasms between those who support these issues and those who vehemently oppose them. In this section, the aim is to summarize and critique several important theoretical contributions that seek to explain how moral disagreement becomes manifest.

\section{MacIntyre: Incommensurable Moral Goods}

Moral Philosopher, Alasdair MacIntyre (1981) believes that we are living in an era of moral disorder, which is characterized by extensive, unresolvable moral disagreement. He writes, “The most striking feature of contemporary moral utterance is 
that so much of it is used to express disagreements; and the most striking feature of the debates in which these disagreements are expressed is their interminable character” (p. 6). Through a historical analysis of the evolution of morality from a philosophical perspective, MacIntyre purports that the current state of moral disorder may be attributed to its decontextualization. That is, over time we have inherited isolated, fragmented, conceptual remnants of morality, which now characterize our moral discourses and evaluations. Thus, moral concepts such as Aristotle’s justice, Kant's universality, and Mill’s utilitarianism have been commingled into a stew of generic moral abstractions, which are now devoid of their original, contextualized meanings and purposes. In other words, we treat our philosophers as contemporaries and fail to recognize that a particular time and space influenced their ideas. The result is an incoherent morality that favors disparate moral goods and leads to widespread disagreement.

MacIntyre claims that morality cannot be a rational enterprise because moral disagreements, viewed as competing moral goods, are incommensurable when they are pitted against one another. Thus, there is no objective measure that concludes that concerns about justice outrank concerns about survival or that issues pertaining to equality are more salient than liberty; yet, we attempt to make moral judgments as if there are impersonal and objective criteria. He attributes this fallacy to the Enlightenment project, which attempted to identify a rational morality but failed and, consequently, exacerbated the problem by leading us to believe that impersonal criteria can be obtained. Over time, the potpourri of moral conceptions has led to a contemporary morality that embodies an implicit emotivism and emphasizes the individual. However, MacIntyre challenges the emotivist's claim, which purports that evaluations and moral judgments 
are “...nothing but expressions of preference, expressions of attitude or feeling, insofar as they are moral evaluative in character” (p. 12). Although he recognizes the presence of emotivisim, he views emotivism as more of a historical condition rather than an inherent truth. To free ourselves from this emotivist state, MacIntyre suggests that we create a coherent approach to morality by referring to the philosophy of Aristotle as a model. A neo-Aristotelian approach includes virtue ethics, which places an emphasis on the development of character and practical wisdom within a local context in which there are clear roles, duties, and traditions. Additionally, there is a teleological ${ }^{1}$ belief that the function of these ethics is to move man from an untutored "man-as-he-happens-be" condition towards a tutored "man-as-he-could-be-if-he-realized-his-telos.”

Although there are other scholars who support the notion that competing moral goods and local conditions affect moral judgments (Posner, 1999; Shweder, Much, Mahapatra, \& Park, 2003), MacIntyre has been criticized for his pessimistic views of moral discord and conceptions of moral change. Churchland (1998) believes that moral disagreement is not due to a disintegration of morality but, rather, is influenced by a changing society that must confront new moral issues as it evolves. He claims that moral progress consists of gradual change and development as it is shaped by a collective life. This progress is akin to scientific progress being that it is an accumulation of collected and recorded social experience that seems to build on a collective socio-moral knowledge.

It can also be said that as society changes, we experience new dilemmas associated with the development of modern technologies and the evolution of conceptual meanings. For example, modern technologies enable us to sustain the life of persons in 
vegetative states, clone animals, and engage in massive exchanges of multimedia products. These changes in our society are often accompanied by a set of outcomes that require us to rethink former concepts or to create new ones (e.g., How do we define 'person'? Who has the right to make decisions that will sustain or end such a life? Is downloading music without the consent of the artist stealing?). As our social context evolves, we are placed in a position that requires us to refine our moral concepts as we apply them to new situations.

MacIntyre uses the phenomenon of moral disagreement to reject the proposition that an objective morality exists. Other scholars agree with this line of reasoning (see Gowans, 2000 for further discussion). For example, legal scholar Richard Posner (1999) argues that morality is largely influenced by local, material conditions. He writes: Every society, every subculture within a society, past or present, has had a moral code, but a code shaped by the exigencies of life in that society or that subculture rather than by a glimpse of some overarching source of moral obligations. To the extent that it is adaptive to those exigencies, the code cannot be criticized convincingly by outsiders. Infanticide is abhorred in our society but routine in ones that cannot feed all the children that are born. Slavery was routine when the victors of war could not afford to feed or free their captives, so that the alternative to enslaving them was killing them. Are infanticide and slavery "wrong” in these circumstances? Is it provincial to say that "we are right about slavery, for example, and the Greeks were wrong,” so different was slavery in the United States until the end of the Civil War and so different were the material conditions that nurtured these different forms of slavery. To call infanticide or slavery 
presumptively bad would be almost as provincial as unqualified condemnation. The inhabitants of an infanticidal or slave society would say with equal plausibility that infanticide or slavery was presumptively good, though they might allow that the presumption could be rebutted in peaceable, wealthy, technologically complex societies. (p. 19)

Although this is a powerful statement in terms of depicting diverging moral judgments relative to specific local conditions, it does not necessarily argue against the case for an objective morality. As Nussbaumn (1993) proposes in her defense of non-relative virtues, in order to determine which virtues are objective we must observe how human experiences overlap as a starting point. Then we must recognize that people aim for a particular good within a particular social context. Similarly, the initial challenge in understanding how moral disagreement becomes manifest is to look beyond the actions in themselves (e.g., slavery, infanticide, etc.) in order to collect a substrate of elements that inform moral judgments within a context of local influence. This substrate of elements, I will argue later, may provide a structure for how we reason about morality.

\section{Shweder: The "Big Three” of Morality and Diverging Moral Codes}

In an analysis of moral discourse among 47 Brahmans living in the city of Bhubaneswar; Shweder, Much, Mahapatra, and Park (2003) categorized the responses into three general moral themes, which has become known as a theory of the "Big Three" of morality. This theory suggests that conceptions of moral goods (i.e., what is perceived to be morally admirable and virtuous) in a given society can be clustered into the following three categories: 
Autonomy. The ethic of autonomy includes concepts of harm, justice, and rights. This ethic emphasizes the individual and promotes individual will and the pursuit of personal preferences. Being a person shapes one's obligations. Protecting the freedom of speech is an example of an ethic of autonomy.

Community. The ethic of community includes concepts of interdependence of persons, hierarchical schemes within the social order, and duty. One’s obligations are shaped by one's role in maintaining and protecting the integrity of the collective (i.e., family, community, nation, etc). The obligations and duty one has as a father in supporting and protecting his family is an example of an ethic of community.

Divinity. The ethic of divinity includes concepts of the natural or sacred order, sanctity, and tradition. One has an obligation to protect souls, spirits, particular spiritual conceptions of humans, and the natural order from disgrace or degradation. The concepts of sin or engaging in acts of purity or spiritual cleansing are examples of an ethic of divinity.

Shweder et al. (2003) claim that these clusters co-exist in discourses of morality and can become manifest in any area of experience including health, suffering, ethics, psychological development, etc. They found that the Brahman moral responses were often characterized by more than one ethic. For example, the idea of a woman staying home to play cards with her friends while her husband cooks rice is a moral transgression characterized by the ethics of divinity and community in Brahman society. In other words, this transgression represents a breach in terms of fulfilling social roles and challenging the husband's earned respect as a "moving God.” He claims that while some 
goods can be foregrounded and made salient, (e.g., autonomy in the United States), others are backgrounded (e.g., autonomy in Hindu society). When an ethic is backgrounded in moral discourse, it does not mean that the society has no use for such an ethic. Rather, the ethic is absorbed into the other discourses (e.g. autonomy as it is absorbed into discourses of community and divinity) and made less explicit.

Shweder et al. (2003) claim that a moral dilemma is created when there is a conflict of moral goods (e.g., duty vs. individual rights) or when there is a conflict of cultural ideologies as represented by diverging moral rationalities. By “diverging rationality,” Shweder et al. (1987) make the claim that there may be more than one rational moral code. They describe the idea of "divergent rationalities" as assuming the following premises:

(1) There exists more than one rationally defensible moral code. (2) In any moral code with rational appeal, some concepts are "mandatory"; without those concepts the code loses it rational appeal. Other concepts are "discretionary"; they permit replacement by alternative concepts whose substitution into the code would not diminish its rational appeal. (3) Every moral code that is rationally defensible is built up out of both mandatory and discretionary concepts. The rational appeal of a moral code would be diminished, it would become empty, if it were divested of all discretionary concepts... (p. 18) Within this perspective, all rationally-based moral codes can share mandatory features but vary in discretionary features.

In a critique of Kohlberg's theory of moral development, Shweder et al., (1987) claim Kohlberg's theory represents one, but not the sole, example of a rational morality. 
In their analysis, they find that the mandatory features of Kohlberg's theory are "the idea of natural law, the principal of harm, and the principle of justice” (p. 18). They also identify six discretionary features, which include the following:

... a conception of natural law premised on natural "rights"; a conception of natural law premised on "volunatarism," "individualism," and a "prior to society" perspective; a particular idea of who is a "person"; a particular conception of where to draw the boundaries around the "territories of the self"; a conception of justice in which likenesses are emphasized and differences are overlooked; and finally, a rejection of the idea of divine authority. (p. 18-19)

Shweder et al. (1987) hypothesize that few people around the world reach a Kohlbergian post-conventional stage ${ }^{2}$ of justice reasoning, because there may be alternative forms of post-conventional reasoning that emerge from different, but equally rational, moral codes. For example, a post-conventional mode of thinking in India is represented by natural “duties” as perceived from a Hindu dharma cultural worldview. In this case, a discretionary feature between Kohlberg’s depiction of a rational morality and the rational morality of a Hindu is in the belief of a divine authority.

The collectivist versus individualistic premises of Shweder's theory have been challenged by Turiel and his colleagues (Turiel, 2002; Turiel, Hildebrandt, \& Wainryb, 1991). For example, there is disagreement about whether or not certain clusters of ethics are foregrounded or backgrounded as a result of cultural worldview. Turiel et al. (1991) found that moral judgments within a specific culture can be heterogeneous and vary across different domains. In other words, rather than to judge social issues via a predominant stereotypic cultural orientation (i.e., such as "individualistic" societies more 
often make rights-based decisions); the study found that the justifications for judgments varied to include orientations that may be thought of as "collectivistic," "libertarian," "traditional," "authoritarian," etc. In addition, Turiel (2002) argues that within social hierarchies across diverse cultures one finds that those at top of the social hierarchy often provide different moral justifications for their actions than their subordinates. Those at top tend to use language that represents autonomy and individual rights. Thus, Turiel believes that there are problems associated with classifying cultures according to a predominant ethic.

Although Shweder's theory has been criticized, one important aspect of Shweder et al's (1987) work is that they have been able to empirically show how cultural conceptions influence moral judgment. In a study that asked both Americans and Brahmans to make judgments about such actions as eating beef, widows eating fish, and addressing the father by his first name, the results revealed that Americans tended to view these actions as social conventions while the Brahmans viewed them as moral transgressions. Thus, different groups can witness the same phenomenon and, yet, form diverging conclusions as a result of interpreting phenomena from different perspectives framed by culturally specific beliefs about the natural world, the psychological, and the afterlife. However, Turiel (2002) states that we must be careful not to conclude that this is evidence that there is more than one moral code. "Differences in such assumptions can give the false appearance of differences in moral judgments” (p. 171). Thus, while people may have seemingly divergent views of the same phenomenon, these views may not be different if we consider them from the perspective of forming general conclusions about harm, rights, welfare, and justice. 


\section{Turiel: Socio-cognitive Domain Theory}

Turiel et al. (1991) view moral disagreement as a result of competing informational assumptions and domain conflict. Socio-cognitive domain theory suggests that, under the course of development, we construct distinct, qualitatively different categories of social knowledge, which include moral, social convention, and personal jurisdiction understanding (Nucci, 2001). In other words, people interpret social situations and apply domain knowledge as they formulate judgments. Accordingly, each domain is demarcated by specific criteria that allow us to identify prototypical examples in the absence of conflict. For example, the moral domain is concerned about concepts or issues pertaining to welfare, harm, justice, and rights; and unprovoked murder, rape, and theft are examples of prototypical moral issues. Within this domain, morality is prescriptive, generalizable, nonalterable, obligatory, and applicable across social contexts. It is not contingent on authority, rules, or tradition.

Social convention knowledge develops as we interact with other members of society and are subjected to agreed-upon rules, uniformities, and other socially constructed meanings. Understanding these norms enables us to function in a predictable, structured social space and helps us to maintain social order. Conventions are closely tied to social context and influenced by authority, rules, and tradition. Some prototypical examples of social conventional norms are mode of dress, greetings, table manners, and rules of the road.

Issues pertaining to the personal domain have to do with an understanding of what falls within the jurisdiction of personal choice. These issues are considered to be the prerogative of the self and entail preferences and personal choices that are not subject to 
social regulation. Although domain theorists consider the personal domain to be culturally universal, the boundaries that delineate what legitimately falls within the personal domain is believed to vary by culture (see Smetana, 2005; Turiel, 2002 for reviews). In mainstream America, some prototypical examples of personal domain issues include choice of friends and privacy. For instance, if a teenager discovers that her parent rummaged through her personal belongings, she may feel angry because she believes that her parent wrongly assumed the authority for invading her privacy.

According to this theory, there are situations that involve more than one domain resulting in a phenomenon called domain overlap. For example, in some situations, people may be required to coordinate a mixture of domains, such as the moral and conventional domains, within a single issue. Under these circumstances, there is a juxtaposition of competing concepts. For example, raising one's hand in class may be viewed as a rule for establishing social order or as an issue of fairness (Smetana, 1983). In addition, there are "nonprototypical” or "multifacted" issues, which include seemingly diverging and controversial issues like abortion, homosexuality, pornography, and incest, in which domain conflict is not as apparent. Some of these issues evoke highly charged debates and controversy.

In an effort to develop a greater understanding of the moral disagreement associated with these nonprototypical issues, Turiel et al. (1991) conducted three studies aimed at ascertaining how people (adolescents and young adults in particular) reason and form judgments about nonprototypcal issues. In study 1, participants were divided into two groups based on the judgments they made about nonprototypical issues such as abortion, homosexuality, and pornography. Both of these groups reasoned similarly 
about prototypic moral, and to some extent, personal issues. However, when it came to the nonprototypical issues, it was found that these acts often elicited different justifications than the prototypical acts. For example, those who viewed these acts negatively reasoned about them in moral terms; yet, they did not believe that these acts should be legally restricted. Instead, it was believed that people should be able to make their own personal decisions. Additionally, personal beliefs, such as when life begins or whether or not homosexuality is part of normative or natural model of sexuality, also affected judgments. Finally, conflicts between domains also became evident such as in the conflict that arises between the welfare concerning the life of the fetus (i.e., moral domain) and the well-being or rights of its mother (i.e., personal domain).

In study 2, seniors from two Catholic high schools in California were recruited to determine if their judgments about the nonprototypical issues would coincide with the position of the church and with perceptions of what it means to be a good Catholic. The study revealed that some individuals perceived a discrepancy between their own beliefs and the beliefs of the church, which implied that individuals do not necessarily adhere to same beliefs as the authority of a group. According to the authors, this demonstrates that even people who share a common group affiliation can be divided like the general public when it comes to nonprototypical issues.

In study 3, 87 undergraduates were asked to make judgements about homosexuality and abortion. Beliefs about when life begins influenced how the students felt about abortion. For those who believed that life begins at conception, abortion was viewed negatively. Turiel et al. (1991) found that informational assumptions (a concept that will be further discussed in detail later) play a key role in nonprototypical issues but 
that these issues were inconsistent and ambiguously applied. For instance some of the women who viewed abortion as acceptable changed their views when it was revealed that the purpose of the abortion was to change the sex of the child. In terms of homosexuality, one group of students viewed homosexuality as a matter of personal choice and did not talk about homosexuality in terms of normal or natural sexual orientation. Those who viewed it as part of the natural order, such as homosexuals are “born like that” also viewed it as acceptable. However, those who believed that homosexuality deviates from the natural order and viewed it as abnormal (e.g., counter to what God has intended for men and women) were more likely to believe that homosexuality is wrong.

In sum, moral disagreement became manifest when the participants disagreed about the consequences of an action (e.g., whether or not abortion results in killing) and the fundamental assumptions about a particular issue (e.g., when life begins). Finally, moral disagreements about nonprototypical issues were influenced by perceptions of how the issue relates to the personal domain. People who viewed these actions as acceptable believed that the issues were a matter of personal choice. However, when there was a juxtaposition of moral with the personal within an issue (i.e., domain overlap), moral considerations were generally more salient than personal domain considerations.

One interesting result of this set of studies is that moral disagreement is viewed as more than a simple conflict of moral goods. In other words, moral judgments appear to involve the coordination of social norms and culturally constructed meanings, personal choice, moral obligations, and outcomes. When these structures are harmonious (as they are depicted by domain-specific, prototypical acts), there appears to be a greater 
homogeneity in responses. However, when there is domain conflict, such as concerns about what is perceived to be a legitimate personal choice versus a moral obligation, the responses tend to be more heterogeneous even within a cultural group that shares a similar, fundamental worldview, (e.g., the Catholic group in Study 2).

Another result of the study worth noting, though minimally addressed by the authors, is the role of intentionality. Turiel et al. (1991) observed that judgements holding that abortion is a matter of personal choice were not consistent across situations. For example, among those who supported a woman's right to choose, $83 \%$ (25 of 30) shifted their stances when asked if abortion is acceptable for the purpose of choosing the sex of the child. The concept of intentionality also became salient when the participants who initially argued against abortion later changed their minds when considering cases of rape or incest victims. In addition to abortion, intentionality was considered in the homosexuality situation as well, particularly in terms of whether or not homosexuality is considered to be volitional. These observations suggest that moral judgments take into account whether or not the agent is in control of a situation and responsible for its outcomes.

\section{Haidt: Social Intuitionist Model of Moral Judgment}

A perspective that challenges rationalistic approaches to morality is Haidt's (2001) social intuitionist model of moral judgment; a model that integrates research on cultural influences and biological processes to explain how we make moral judgments (e.g., Greene \& Haidt, 2002). In this model, Haidt argues that moral judgments begin with a quick, automatic intuition for what is right or wrong. By intuition, he means a "sudden appearance in consciousness of a moral judgment, including an affective valance 
(good-bad, like-dislike), without any conscious awareness of having gone through steps of searching, weighing evidence, or inferring a conclusion” (p. 818). Biological processes such as affect, intuition, and specific neurological functions guide these automatic responses.

In the overall model, Haidt de-emphasizes rational thought and claims that reasoning about judgments is typically a post-hoc, social construction. In other words, reasoning is required only when we are put in a position to defend our stances. According to Haidt, "the social intuitionist model is an antirationalist model only in a limited sense: It says that moral reasoning is rarely the direct cause of moral judgment” (p. 815). Instead, these intuitive judgments are largely influenced by affect. Although this model downplays the causal relationship between reasoning and judgment within the individual, it does acknowledge that a causal relationship is more likely to occur between people in social situations. However, Haidt proposes that we do not persuade other people to our point of view as a result of logic, but rather, as a result of triggering a new affective valence in others. In addition, our judgment is likely to be shaped by group norms, particularly when it comes to groups that are salient to us, such as associations that provide friendships or social allies. Although it is rare, it is hypothesized that people may override intuitions through the use of logic or private reflection by activating a new intuition that contradicts the initial intuitive judgment (e.g., as in the case of looking at a dilemma from more than one perspective). However, this is believed to happen only when the dilemma requires the brain to engage in more effortful processing. Otherwise, the brain will attempt to conserve its resources by resorting to default intuitive responses. 
Haidt's theory suggests that the source of moral disagreement is the result of competing intuitions. This conflict of intuitions can lead one to experience a greater moral challenge. He states:

A person comes to see an issue or dilemma from more than one side and thereby experiences multiple competing intuitions. The final judgment may be determined either by going with the strongest intuition or by allowing reason to choose among the alternatives on the basis of the conscious application or a rule or principle. This pathway amounts to having an inner dialogue with oneself. (p. 819)

Thus, in the example of an issue rife with conflict, such as abortion, one may intuitively feel that abortion is wrong when considering the fetus, but then may intuitively feel that abortion is acceptable after reflecting on the mother's perspective. According to Haidt, under these circumstances, one determines whether or not abortion is acceptable by either resorting to reason or relying on the strongest intuition. Extrapolating the conflict of intuitions to an intrapersonal context is likely to be the source in moral disagreement. In other words, moral disagreement is the result of conflicting intuitions between various people.

Although the social intuitionist model has several strengths including the integration of affect, automaticity, and a strong social component; this model seems to overemphasize the role of affect. Pizzaro and Bloom (2003) contend that this model fails to recognize how we educate our moral intuitions through the use of cognitive appraisal and second-order emotional control. Through cognitive appraisal, the individual can appraise the situation and shift his perspective. For example, a police officer may be 
angry at a motorist for disregarding the traffic signals; however, his anger may turn to sympathy as he discovers that the motorist is about to have a baby and needs to get to the hospital as quickly as possible. Additionally, as a result of second-order emotional control, the individual can control impulsive, affective reactions by choosing how to redirect her focus. For instance, one can avoid an uncomfortable situation by purposefully attending to something that is more pleasant. Finally, Pizarro and Bloom also believe that real-life dilemmas often require us to deliberately reason in ways that are not subject to quick, automatic responses. Some of their examples include asking any of the following questions: How much should I give to a charity? What is the proper balance between work and family? What are my obligations to my friends? In sum, Pizarro and Bloom take the stance that, "Our immediate moral intuitions can be (and are) informed by conscious deliberation, and this deliberation plays a central role in our moral judgments” (p. 195).

In his response to Pizarro and Bloom, however, Haidt (2003) claims that his model is equipped to account for sudden changes in context and argues that we need to free ourselves from the notion that there is a direct causation between belief and moral judgment. For example, in a criticism of the Turiel et al. (1991) studies, Haidt (2001) writes:

The correlation [Turiel et al.] found between judgment and supporting belief [i.e., informational assumptions] does not necessarily mean that the belief caused the judgment. An intuitionist interpretation is just as plausible: The anti-abortion judgment (a gut-feeling that abortion is bad) causes the belief that life begins at conception (an ex post facto rationalization of the gut feeling). (p.817) 
Earlier, it was noted that some of the abortion responses in Turiel et al.'s (1991) study shifted according to what appeared to be new perceptions of intentionality and responsibility (i.e., some judgments changed when asked to consider abortion in the case of rape or incest or for the purpose of choosing the sex of the child). According to Haidt's model, it is unlikely that reasoning about intentionality and responsibility had anything to do with the change in these responses. He claims, "the [social intuitionist] model is quite explicit that the moral judgments change when a situation is suddenly viewed in a new light and new intuitions are triggered” (Haidt, 2003, p. 197). Thus, his model would suggest that the shift in abortion responses did not result from reasoning about new information, but rather, the change in judgment was due to the triggering of new intuitions in a different context. However, it should be noted that we often make interpretations about intentionality before we experience an emotional response (e.g., Did that man clip my shoulder on purpose or was it an accident?) (Weiner, 1985, 2001). To argue that our "gut-feelings" about a given issue (e.g., abortion) will vary according to different contexts intuitively without the accompaniment of some level of reasoning seems unlikely because recognizing how contexts change from one situation to the next requires the mind to process a transformation of information.

Though Haidt is correct to note that some moral judgments are automatic, quick, and effortless; it may be false to suggest that moral reasoning has nothing to do with the formation of quick moral judgments. Many skills require repeated practice before they can become automatic or result in quicker response times (Pashler, Johnston, \& Ruthruff, 2001). For example, learning to drive a car with an automatic transmission requires the driver to think about how and when to shift gears in accordance to a given speed limit 
and direction. After repeated experiences, shifting gears becomes automatic and requires less deliberate thinking. Analogous to this example, it can be hypothesized that children engage in reasoning about morality before it becomes more intuitive and automatic. When a young child says, “That's not fair,” after observing that her older sister is allowed to stay up later than she; the child is making a moral observation about the world from a fairness-means-equal-treatment perspective. After her mother explains that young children require more sleep than older people in order to be healthy, she is now introduced to the concept of fairness-can-result-in-unequal-treatment. After additional experiences, such as giving the youngest children a head-start in a race or allowing for persons in wheelchairs to board a plane before others, the child gradually learns how fairness can mean different things under different contexts as she actively constructs and fine-tunes this knowledge. This is similar to what Aristotle (1999) refers to as the development of practical reason, which is the product of maturation, moral education, and experience. Aristotle believes that practical reason enables one to continuously aim toward the good (i.e., to make prudent ethical decisions) within a social milieu. Over time, with the aid of proper training and opportunity to practice, these responses become automatic.

\section{Churchland: the Moral Network Theory}

In his moral network theory, philosopher Paul Churchland (1996; 1998) uses research in neurobiology to create a theory of metaethics. He contends that, in the physical world, specific skills are acquired as a result of training neuronal networks to respond to sensory input. Likewise, we acquire moral knowledge the same way; we 
develop skills of perception, manipulation, recognition, and behavior that enable us to navigate the social world.

Although Churchland does not explicitly define morality, he suggests the ability to discriminate between right and wrong is an essential component of moral judgment. In the process of acquiring moral knowledge, neuronal networks are trained to partition an abstract, multidimensional, conceptual space into hierarchical categories that discriminate between relevant categories (e.g., “morally insignificant” vs. “morally significant”). Furthermore, like cases are clustered together and contribute to the development of a central "hot spot" that represents a prototypical instance of that category. More specifically, Churchland (1998) states that within this abstract space of possible neuronalactivation patterns, we construct a:

...conceptual space for moral representation, and it displays an intricate structure of similarity and dissimilarity relations; relations that cluster similar vices close together and similar virtues close together; relations that separate highly dissimilar action categories into spatially distant sectors of the space. This highdimensional similarity space... displays a structured family of categorical "hot spots" or "prototype positions" to which actual sensory inputs are assimilated with varying degrees of closeness. (p. 86)

Thus, if the stimulus resembles any of the clustered vices that constitute the "morally bad" category, the stimulus is likely to be interpreted as reflecting that category due to its close proximity to the position of the prototype in the conceptual space. In sum, moral perception is believed to involve the activation of neuronal patterns that closely resemble the prototype as information is assimilated to the nearest of the available prototypes, 
which were created via prior training. In some cases, the stimuli may be interpreted as being ambiguous or it may activate more than one prototype. Churchland believes that these are the sources of moral disagreement, and in any moral argument, one person attempts to elicit a similar activation pattern in the other person. According to Churchland, as a result of moral development, children generate perceptual and behavioral prototypes, which require repeated exposure and practice of relevant moral categories. These prototypes are continuously readjusted, particularly in the aftermath of experienced failure.

One problem with this framework is that Churchland's prototype model may not be a true prototype model as it is defined by scholars who study concepts and categorization (Markman \& Gentner, 2001; Medin, 1989; Medin \& Smith, 1984). According to this body of research, there are currently two predominant categorization models: the exemplar model and the prototype model. As opposed to classical views of categorization, which hold that categories have distinct boundaries that consist of a set of fundamental characteristics that are common to all members in the category, the exemplar and prototype models depict categories as "fuzzy" or ill-defined. Therefore, category membership is based on a correlation of attributes that characterize typical instances of that category. For example, the classical view holds that boots have a set of characteristics that clearly distinguish them from shoes. In this view, boots have unique properties that shoes lack, such as height. However, in reality, we know that some footwear resemble both shoes and boots. The exemplar and the prototype models are better equipped to account for these unclear cases. In other words, these models 
determine which category best represents the stimulus based on the assumption that better members will share more characteristic properties than poorer ones.

What differentiates the exemplar model from the prototype model is in how mental representations are structured. The prototype model assumes that, as one accumulates experience with the examples of a category, a summary representation is formed as a result of abstracting out the central tendency of salient properties. This summary or ideal representation becomes the prototype, which is attributed as having the most characteristics in the category. Stimuli are compared to the prototype, and those with highly correlative features are likely to be classified as a member of that category. The exemplar view, on the other hand, holds that there is no summary representation. Instead, a category is represented by multiple exemplars. Thus, any new stimulus will be compared to other known examples of that category. For instance, if a grocer suddenly encounters novel produce and does not know whether to categorize it as a fruit or as a vegetable, she would engage in a process that compares the unidentified produce to stored examplars of fruits and vegetables. If the novel produce more closely resembles a particular fruit, say a banana, and does not closely resemble any of the vegetable exemplars, then it will probably be categorized as a fruit.

The reason why Churchland's theory may not represent a true prototype model is that it does not explicitly identify the abstracted properties of what is morally bad or morally good. In an analogy using the classic example "bird," several hierarchical categories are constructed as relevant to identifying birds. These categories may include “animal” vs. "not animal”; "bird” vs. "animals that are not birds”; and within the category "bird," specific birds like robins, chickens, sparrows, and hawks. According to the 
prototype model, the mind will abstract the central tendency of properties of "bird" (e.g., sings, wings, flies, lays eggs, etc.) and the summary representation of these attributes will become the prototypical bird. As a result, exemplars that share more characteristics with the prototype will be closer to the prototype than others. For instance, a robin may be a better representation of a prototypical bird than a penguin.

In Churchland’s model, hierarchical categories are also constructed: “morally significant” vs. “morally insignificant”; “morally bad” vs. “morally praiseworthy”; and within “morally bad” actions like lying, cheating, and betraying. However, unlike the previous bird example, Churchland does not identify the abstracted characteristics of the morally bad or morally good prototypes, which makes it more akin to the exemplar model. Churchland claims that like actions (such as lying, cheating, and betraying) represent a category as a cluster of virtues or vices. Relying on actions like lying, cheating, and betraying to represent the "morally bad" category may not be a reliable means for categorization, particularly since there are times when these actions can also be viewed as morally good actions (see Flanagan, 1996 for a discussion on lies). For example, one could lie to an aggressor in order to save the life of his victim or lie to a friend in order to plan her surprise party. These types of lies are qualitatively different than lying to cheat someone out of his money. Therefore, it is important for a theory of moral prototypes to identify the abstracted properties of "morally bad" and "morally good” in order to develop a greater capacity to predict how people will respond to and discriminate between particular situations. 


\section{What Can We Learn From these Scholars?}

Each of the scholars is noted for his contribution to moral psychology or philosophy. Although, at first glance, some of these theories appear to be qualitatively different from each other, three general themes emerge from this review:

1. A good theory of moral disagreement must consider the role of conflict. MacIntyre and Shweder both point to competing moral goods or obligations as sources of moral disagreement (e.g., justice vs.survival, duty vs. rights). In socio-cognitive domain theory, Turiel argues that there is conflict or overlap between the moral, personal, and/or social convention domains. Finally, Haidt argues that moral disagreement becomes manifest when there is a conflict of intuition, while Churchland believes that a conflict of moral prototypes is the source of moral disagreement. Thus, a new theory of moral disagreement must cogently identify the salient elements that lead to conflict. It may be possible that some issues contain more than one type of conflict (i.e., a conflict of moral goods and a conflict between moral and personal concerns in a single issue).

2. A good theory of moral disagreement must be able to account for cultural differences. As previously mentioned, there is considerable debate about whether or not multiple moral codes exist or if there is a universal moral code shared by all people. Shweder and Turiel point to the importance of understanding of how worldviews can affect moral judgments, particularly when people are making diverging judgments about the same phenomenon. In other words, what people believe to be true, valued, virtuous, and desirable 
affects how a situation is interpreted and can result in moral disagreements between people who hold different informational assumptions or worldviews. Thus, new theories of moral disagreement must include explanations of how culture-specific informational assumptions influence moral judgment.

3. A good theory of moral disagreement must be able to explain how some issues result in quick moral judgments while others require more deliberative thinking. As indicated by both Haidt and Churchland, there are cognitive dimensions that enable us to make quick, effortless moral judgments.

\section{The Properties of Moral Prototypes}

Building on the work of Churchland and the criteria listed above, it is important to identify what the abstracted properties of a moral prototype are and explain how the interaction of these properties lead to moral disagreement. This dissertation proposes that there is a structure to morality in which salient properties are used to reason about morality and make distinctions between what is morally praiseworthy and morally blameworthy. A review of the empirical research suggests that people tend to focus on several salient dimensions when making moral judgments.

\section{Social Context}

Beliefs about what is sacred, desirable, true, virtuous, and beautiful are socially constructed and transmitted as local knowledge within a particular time and space (Geertz, 1973; R. Shweder, Balle-Jensen, \& Goldstein, 2003; Shweder, 1984, 2003a). The knowledge we gain from this social experience influences how we interpret situations and has a bearing on judgments and behaviors. Within the social context, we ask and respond to a wide array of questions such as, What is a person? When does life 
begin? When does one become an adult? Is there an afterlife? What does it mean to live a quality life? What is the best way to raise children? Do we have an obligation to take care of the elderly? The narratives that emerge from such questions can vary across cultures, subcultures, and even generations; resulting in different interpretations of phenomena as shaped by perceptions of a natural order, the metaphysical, history, science and technology, economic systems, political systems, religion, and the environment. Thus, beliefs about how one should or ought to live can vary according to social context as experienced by people within a particular time and space.

In some instances, diverging beliefs about what is sacred, desirable, true, virtuous, and beautiful can lead to highly contested debates about issues such as gay marriage, abortion, and female genital circumcision. For example, debates about female genital circumcision (FGC) often involve two very different perspectives concerning what is believed to be "true" about the practice. Some scholars believe that FGC poses physical dangers, oppresses women, has a negative impact on women’s sexual functioning and ability to experience pleasure, and is a coercive action that is often inflicted onto those who are too young to fully consent (Nussbaum, 1999; Okin, 1999). Others scholars believe that FGC does not pose serious health risks; symbolizes a rite of passage for young women; and is tied to notions of beauty, femininity, strength, and psychological well-being (Leonard, 2001; Shweder, 2003b).

Not only do knowledge conflicts become salient in highly controversial issues, but one can also observe how the social context has an impact on the taken-for-granted decisions we make in our daily routines. For instance, beliefs of what is sacred, desirable, true, beautiful, and virtuous provide a framework for how we arrange our 
sleeping spaces. In a study that compared the sleeping preferences of informants in Orissa, India and Hyde Park, Illinois; Shweder, Balle-Johnson, and Goldstein (2003) found differences in how the informants extract moral meaning from a sample of hypothetical sleeping arrangements. The informants in Orissa were more likely to endorse co-sleeping arrangements with children and tolerated the separation of mother and father at bedtime. Principles associated with chastity anxiety, hierarchy, and protection of the vulnerable emerged from the Oriya responses, while the middle-class Americans were more concerned about fostering independence and self-reliance in their children and promoting a healthy marriage as signified by parents who share the same bed in a room apart from the children.

According to Shweder (2003a), cultural knowledge becomes embodied and "constitutive of (and thereby revealed in) a way of life" (p. 11). Thus, the influence of the social context involves more than the mere location of an individual, as moving from one environment to another does not necessarily lead to a transformation of these beliefs. Reverting back to the sleeping arrangement example; in the United States, AsianAmericans $^{3}$ are approximately three times more likely to co-sleep with an infant than whites (Willinger, Ko, Hoffman, Kessler, \& Corwin, 2003) even though some groups warn parents of the dangers associated with Sudden Infant Death Syndrome and suffocation (McKenna, 2002). Thus, cultural practices and beliefs can be strong and persistent despite external pressures to change.

Anthropologist Clifford Geertz (1973) believes that abstracting humans from their social context only renders them as arbitrary caricatures which leads to a gross misrepresentation of human life. From this perspective, Geertz claims that empty 
universals such as "marriage," "religion,” or "property” do not represent the same content from culture to culture and do little to add to our understanding of how people conceptualize reality and the practices manifested in this reality. For example, to say that "all people marry" is misleading because the term "marriage" imposes ethnocentric meaning onto populations that may not necessarily share the same concept. Additionally, it is bereft of content, because it does not provide us with information about what marriage means under different contexts. That is, it does not tell us anything about the conditions and circumstances that lead people to prefer one type of relationship to another (e.g., preferences for polygamous relationships vs. monogamous relationships). Because there is such a strong interconnection between human life and context (i.e., man shapes culture and culture shapes man), new theories of morality must take into account how local knowledge influences moral judgments.

A body of empirical studies has been conducted to develop an understanding of the role of local knowledge in the formation of moral judgments (Turiel et al., 1991; Cecilia Wainryb, 1993; Cecelia Wainryb \& Ford, 1998). In this context, local knowledge is referred to as informational assumptions. Turiel (2002) states:

People’s assumptions about reality, which come from various sources, also must be taken into account in understanding how they come to decision. When applied to moral and social decisions, such assumptions function as an informational kind - what I will refer to as informational assumptions.... Informational assumptions are not solely particular facts derived directly from some kind of data-gathering process. Such assumptions can be derived from conceptual systems and theories. However, knowledge derived from conceptual systems - scientific or otherwise - 
is used in an informational-factual sense (often with ambiguities) in situations involving moral judgments. (p. 143-144)

In other words, these facts manifest from a reality or worldview as experienced in a particular social context. For example, informational assumptions about when life begins and what constitutes a person have been found to bear on how adolescents and young adults reason about abortion (Smetana, 1981; Turiel et al., 1991). Additionally, in a study that asked sixth grade, tenth grade, and undergraduate university students to evaluate acts that potentially included harm or injustice, Wainryb (1993a) found that a significant number of participants changed their evaluations after these acts were depicted under different circumstances and the informational assumptions were made apparent. For instance, some students said it was unacceptable for a father to spank his child in order to make himself happy. However, they judged spanking as more acceptable when they learned that the father believed it was the only way to remove an evil spirit from a misbehaving child.

While it is tempting to argue that different perceptions of what is true and real supports the cultural relativist's claim, one must not jump to that conclusion. Wainryb (1993b) believes that informational assumptions "bear on the interpretation or meaning attached to the event in such a way that if differences were to exist in informational judgments (e.g., in what two indivduals or two cultures believe to be true), different meanings would be attached to the same event” (841). However, she argues, these differences are only made at the information level and do not necessarily represent differences in the moral domain. 
In sum, the social context influences how a situation will be interpreted via a culture-, subculture-, or generation-specific lens. The social context shapes our notions about what is sacred, desirable, true, virtuous, and beautiful, which leads to prescriptions about social roles and obligations in terms of what one should or ought to do. These notions coalesce into a worldview or an ideology which informs our moral decisions and provides a framework for identifying the virtues and vices in a given society (Lakoff, 2002); thus, moral prescriptions, in a given social context, often assume valences of right or wrong. For example, some people may believe that it is virtuous to marry for love or wrong to place a parent in a nursing home. Although social prescriptions have a strong bearing on moral judgment, they, in themselves, are not the only salient factors in moral judgment making and do not function in isolation.

\section{Autonomy and Consent}

Imagine the following scenario:

Stan is visiting his doctor for a routine physical. The doctor administers a number of tests, updates Stan's immunizations, and tells him that everything appears to be in order - he is in perfect health. However, after he leaves the office, the back of his upper arm starts to itch and when he scratches it, he notices something very small, hard, and grainy under his skin. He returns to the office and tells the nurse about his problem. She responds, “Oh that's just your identification and tracking device. We started a program in which we implant medical nanocomputers in all of our patients. Not only are we able to monitor your vitals and track your location, but we can also provide real-time medical records in case of an emergency." Stan cannot believe what he is hearing and orders the nurse to remove the device from his skin at once. 
The nurse tells him that it is impossible to remove the device once it has been implanted. Stan is outraged. Was it wrong or all right for the doctor to implant this device in Stan's arm without his consent?

Although Stan’s scenario is fictional, there are many real-life moral issues in which the lack of consent has spurred serious debates. Was it wrong to force JapaneseAmericans into determent camps during World War II? Is it wrong to force students to acquiesce to school-initiated drug testing? Should businesses be required to explicitly communicate how a customer's personal information will be used? Should subjects be warned of the potential risks of participating in a research experiment? In some cases, whether or not the target of an action consents to the action has a bearing on how we judge the moral issue.

One premise of this paper is that morality is a social phenomenon that functions to promote cooperation and survival, build relationships, and foster the physical, mental, and spiritual well-being of groups and individuals (Kohlberg et al., 1983; Krebs et al., 2002; Pinker, 2002; Shweder et al., 2003; Waal, 1996). In this view, morality is a system of interpersonal relationships that is "other” regarding (Nucci, 2001; Nucci \& Weber, 1995), and moral situations require us to think about how actions directly or indirectly affect others. In many social circumstances, an agent initiates an action that affects a target. For example, a commuter may start a conversation with a fellow passenger, a terrorist group abducts two civilians, a doctor uses a patient to test an experimental drug, etc. In some cases, the target (i.e., those affected by the action) accepts or tolerates the agent's actions. However, there are also situations in which the target does not acquiesce while the agent pursues the action against the target's will. Some philosophers argue that 
unjustified force or coercion is immoral and advocate for a certain level of autonomy. For example, Gewirth (1986) believes that freedom and well-being are necessary conditions for action and for successful action in particular; thus, these conditions are important to the conception of fundamental human rights. In his Formula of Humanity, Kant (1785/1998) says, "So act that you use humanity, whether in your own person or in the person of any other, always at the same time as an end, never merely as a means” (p. 38). This principle has been interpreted as emphasizing the respect of rational beings in their ability to make decisions for themselves and to share in deliberations. Using force or deceiving another for the purpose of promoting self-interest manipulates the wills of others and does not allow others to reason freely, thus, resulting in a moral violation against humanity (Korsgaard, 1998).

Presently, there is little empirical research that directly looks at consent and morality. However, there is a body of developmental research that supports claims for autonomous choice and decision-making. Though this research does not fully define what consent ${ }^{4}$ is nor does it identify the characteristics of those who are in a position to consent $^{5}$, the research does reveal and justify an area of personal agency. In other words, it identifies the circumstances under which people perceive the will of the target to be respected. Reverting back to social domain theory, the personal domain has to do with actions that are believed to be outside of the jurisdiction of social regulation (Nucci, 2001; Smetana, 2005; Turiel, 2002). These are activities that do not threaten the welfare of others, pose rights or fairness issues, and fall outside of the socioconventional domain. In a sense, the domain of personal jurisdiction is a constructed boundary between the self and the group concerning a specific sphere of actions, which typically include 
preferences and choices pertaining to privacy, leisure, control over one's body, and friendship. For example, some people believe that actions like choosing one's friends should be left to individual discretion. According to social domain theory, this area of personal discretion does not develop as a result of innate claims for control, but rather, as a result of a construction of the child's claims and the interactions and negotiations of other members of society (Nucci \& Weber, 1995). Affording the self with jurisdiction over decisions about actions in the personal domain is thought to contribute to identity formation, personal integrity, individuality, and agency. Nucci (2001) writes, "the personal represents the set of social actions that permit the person to construct both a sense of the self as a unique social being... and the subjective sense of agency and authorship...” (p. 54). In developmental studies of the personal domain, it has been found that children and adults rarely argue about moral issues and children are more likely to endorse the parent's or teacher's authority when it comes to concerns about welfare, rights, or justice (Nucci, Camino, \& Sapiro, 1996; Nucci \& Weber, 1995; Smetana, 2005). Instead, the majority of parent-child conflicts emerge from disagreements about issues that fall within the personal domain. In discussions about culture and morality, personal freedoms and a conception of the self as a unique individual have been associated with a Western morality that emphasizes rights and entitlements, while non-Western societies have been characterized as cultures that foreground duties and interdependence in their moral discourses (Shweder et al., 2003). However, Deci and Ryan (2000) believe that a certain level of autonomy is a universal organismic need. According to Deci and Ryan, autonomy should not be confused with Western characterizations of independence and individualism. 
Instead, autonomy refers to volition and the freedom to self-organize behaviors and experiences in a way that endorses one's actions and integrates them into a sense of self.

Cross-cultural research on the personal domain supports the notion that the development of personal choice and agency in children is not limited to Western cultures. Thus, while activities categorized as personal vary according to culture and context, there is evidence to suggest that setting limits to what groups may impose onto the individual may be a universal phenomenon. For instance, in Brazil, (Nucci et al., 1996) conducted a study in which lower and middle class children were asked about such personal issues as keeping a diary, keeping a conversation private, hairstyle, and choice of friends. Although younger, lower class children were more likely to make judgments in accordance with norms and authority, by adolescence, there were no class differences and a significant number of both lower and middle class children viewed these actions as personal. Thus, the study revealed that Brazilian children conceptualized a personal domain of actions. Research in China has shown that Chinese adolescents justify reasons for making decisions about family, school, and peer activities (e.g, curriculum, games, movies, tutoring, and field trips) in terms of rights, autonomy, and democratic norms (Helwig, Arnold, Tan, \& Dwight, 2003). Additionally, as with their US counterparts (Nucci \& Smetana, 1996; Smetana, 2005), Chinese and Japanese adolescents and young Chinese children also conceptualize a personal domain (Hasebe, Nucci, \& Nucci, 2004; Yau \& Smetana, 2003a, 2003b). According to Yau and Smetana (2003a), Chinese adolescents were asked to describe the conflicts they most recently had with their parents and reported that most of the conflicts involved disputes about personal jurisdiction. It was found that Chinese adolescents rationalize these conflicts with claims to personal 
autonomy, thus, expressing a desire for freedom, independence, and individuality. Additionally, when comparisons were made between adolescents from Hong Kong and Shenzhen, it was found that Hong Kong adolescents were more likely to view choice of activities as falling within the personal domain. Conversely, in Shenzhen, adolescents were more likely to endorse homework and academics as personal issues. Thus, there is evidence that cultural and contextual variations influence the expression of personal jurisdiction, and consequently, may influence how we differentiate which actions are legitimately subject to norms and authority.

The research on the personal domain suggests that, at times, the goal to protect the welfare of others or to promote justice will legitimatize the control from a higher authority; thus, it is plausible that the consent of an individual or group may not always be warranted, particularly when it involves a moral concern (e.g., public safety). Because issues that fall within the personal domain are perceived to be associated with personal choice and preference, using undue authority to achieve a particular end against the will of the target may lead to perceptions of wrong-doing. In other words, if there is a set of actions that is not subject to social regulation and an agent engages in these actions by using force, deception, or coersion, then it is predicted that the role of consent will bear directly on moral judgments. Thus, identifying an area of personal jurisdiction provides us with an idea of where consent issues are likely to be salient. For example, in a society in which the choice of a spouse is a personal domain issue, members of that society are likely to view forcing a person to marry against her will as a moral transgression.

It should also be noted that some research suggests that forcing one to acquiesce to the will of a controlling agent in areas of personal jurisdiction may result in negative 
consequences. In a study that compared US and Japanese adolescents, it was found that greater parental control over personal issues was positively correlated with self-reported internalized, psychological symptoms (e.g., anxiety, depression, and somatization) (Hasebe et al., 2004). In addition, Barber (1996) found a correlation between depression in preadolescent and adolescent children and a parent's use of psychological control, which includes control attempts that interfere with healthy psychological and emotional development of the child (e.g., intrusion into thinking processes and self-expression). A study conducted by Assor, Roth, and Deci (2004) demonstrated how the use of love withdrawal as a form of psychological control, in which children relinquish autonomy for the purpose of receiving love from their parents, is associated with fluctuations in selfesteem, poor coping skills, feelings of disapproval, and a generalized anger and resentment towards parents. Finally, according to Bruch (1982), people with anorexia nervosa often do not feel in control of their bodies and have difficulty conceptualizing how to live their own lives. Oftentimes, people with severe eating disorders come from homes in which the controlling parents had little regard for their children's desires. It is hypothesized that these negative consequences affect moral judgments via inference or the direct knowledge of these consequences.

\section{Intentions and Outcomes ${ }^{6}$}

When people talk about moral issues, they often ask the question, Why? Why did Michael Schiavo want to take his wife, Terri, off of life support? Why did pop superstar, Michael Jackson, invite prepubescent boys to spend the night in his home? Why did the federal government take so long to respond to Katrina? It is believed that asking "why" is a fundamental human quality that has considerable implications for how we structure 
our lives. Not only do the responses to these questions facilitate our understanding of the environment, events, and human behavior, but they also have a functional capacity to guide future actions and construct judgements of responsibility that promote or thwart particular behaviors (Weiner, 1985, 2001). In the moral domain, answering why questions specifically enable us to make ascriptions about intentionality.

Although, to date, there is no consensus about how to define the specific nature of intentionality, scholars do agree that intentionality, in its general form, is an important feature in moral judgments of blame and responsibility (Malle, Moses, \& Baldwin, 2001; Mele, 2001; Weiner, 2001). For example, moral psychologist, Augusto Blasi (1999) states one condition of moral action is that, "Any moral action should be intentional. It cannot be accidentally produced or outside of the agent's consciousness. It must be a result of reasons" (p. 12). When it comes to specific cases, intentionality will have an effect on moral judgment. For instance, philosopher Owen Flanagan (1996) argues that we can differentiate between practical jokes and lies by looking at the intent of the agent. Though in both cases there is a desire to deceive, the goal of a practical joke is to make fun and not cause harm; the intent of an immoral lie is to disregard others or threaten another's well-being.

Even though intention and intentionality are sometimes used synonymously in psychology, Malle, Moses, and Baldwin (2001) make the following distinction between the terms from a folk-use perspective:

Intentionality....is a quality of actions (those that are intentional or done on purpose), whereas intention is an agent's mental state that represents such actions. This type of mental state often precedes its corresponding action or even occurs 
without it. One can therefore ascribe intention to an agent without making a judgment of intentionality. The reverse is not true, however: The judgment of an action’s intentionality typically implies the ascription of an intention to the agent . (p.3)

In other words, intention is a mental state that integrates desires and beliefs into a reason for acting. Intentionality has to do with the performance of an action that results in particular outcomes. Thus, within the framework of this paper, intentionality concerns whether or not people perceive a property of actions as being done purposefully or intentionally, and intention has to do with the actor's mental state.

In psychology, the areas of theory of mind, attribution theory, and moral development research have produced a body of work relevant to the topic of intentionality. Intentionality is believed to be a universal phenomenon and fundamental to how we perceive and interact in the social world (Povinelli, 2001). Although cultures may differ in how they interpret the context of the action or in how they weigh extenuating circumstances (e.g., whether or not to absolve the agent of responsibility as the result of emotional duress, anger, and immaturity), the evidence suggests that nonWestern cultures also use concepts of intentionality to make moral judgments (Ames et al., 2001; Bersoff \& Miller, 1993; Chiu \& Hong, 1992).

While there is considerable debate about the extent to which infants and nonhuman primates develop concepts of intentionality (Baird \& Baldwin, 2001; Povinelli, 2001; Wellman \& Phillips, 2001), studies have found that these concepts start to develop early in life. Beginning with his classic work on children and moral judgments, Piaget (1932/1965) observed that young children used the severity of an outcome as basis for 
ascribing moral judgment and later transitioned into forming judgments that also included concepts of intentionality. Today, it is believed that children use more than a simple matching rule (e.g., bad outcome = bad intention) and develop an insight about other people’s mental states and intentional behavior before the age of 5 (Moses, 2001; NelsonLe Gall, 1984, 1985). For example, one study (Nelson-Le Gall, 1984) found that 3-yearold children evaluated more harshly a mean agent who causes a foreseeable injury than the same agent who causes an unforseeable injury. This suggests that young children make distinctions between actions that are "done on purpose" from those that are accidental in their moral judgments. Although children under 5 appear to have an emerging concept of intentionality, it is not as sophisticated as adult concepts. Over time, it is speculated that children continue to refine distinctions between intentional and unintentional behavior by developing the ability to conceptualize chance, coordinate the ascription of responsibility to more than one agent (Nelson-Le Gall, 1984), distinguish intention from desire (Moses, 2001; Olthof, Ferguson, \& Luiten, 1989), and use context clues to make inferences about intentionality, such as the level of an agent's intention (i.e., how badly the agent wanted to perform an negative action) (Jones \& Thomson, 2001). Additionally, it has been found that through the course of development, we construct a "blame schema" which represents the relationship between blame, intentionality, and consequence (i.e., blame/punish $=$ bad intent + bad outcomes) (Hermand, Mullet, Tomera, \& Touzart, 2001; Zelazo, Helwig, \& Lau, 1996). Thus, there appears to be a coherent relationship between bad intent, bad outcomes, and moral judgments of blame and punishment. 
As previously mentioned, intentionality influences perceptions of responsibility, blame, and punishment and enables us to distinguish between concepts such as manslaughter and murder. In his theory of social conduct, Weiner (1995) maintains that the perception of whether or not the act was intentional is essential to making moral judgments. According to Weiner, when an act is intentional, the agent self-initiates the action and pursues the act with foresight and knowledge of its consequences.

In order to understand the relationship between intentionality and responsibility, Weiner breaks intentionality into two sub-components: (a) act intentionality and (b) outcome intent. Act intentionality has to do with the purposeful action that is initiated by the agent, and the outcome intent is related to the goal that is tied to the purposeful action. Consider the following examples: (a) a woman drives recklessly and accidentally kills a pedestrian; (b) a woman drives recklessly for the purpose of killing a pedestrian. In both cases, the act intentionality is the decision to drive recklessly. However, what differentiates the two situations is the outcome intent. In the first case, the action and the goal are not connected since the intention was to drive recklessly but not to kill anyone. In the second scenario, the action is initiated in order to produce the intended outcome (i.e., the woman drives recklessly specifically to kill the pedestrian). According to Weiner, it is likely that both agents will be held responsible, since speeding is internal and controllable ${ }^{7}$. Internal, controllable actions are more likely to be punished since they are initiated with a certain level of freedom and choice. However, the agent in scenario B will probably experience a more elevated judgment of responsibility because the action was used to produce the intended outcome of killing the pedestrian. Thus, the varying degrees of responsibility are influenced by whether or not a controllable act is perceived 
to result in an intentional outcome. Generally, the theory posits that people are not judged as harshly when negative outcomes are not intended; though, it may not reduce the judgments of responsibility to zero.

Weiner argues that it is important to recognize that responsibility is not synonymous with attribution. Instead, judgments of responsibility progress from a causal attribution to an inference that is made about the person (e.g., He "should" not have done that. They "ought” to have done otherwise.). In addition, responsibility judgments presuppose human or human-like causality, which may include gods or animals that are perceived to initiate human-like means-ends goals. Thus, inanimate objects that lack agency such as scissors, rocks, or cameras, in themselves, are not perceived as responsible agents for negative outcomes. Finally, Weiner claims that judgments of responsibility are influenced by mitigating circumstances. Mitigating circumstances alleviate or eliminate judgments of responsibility. For instance, mentally ill persons or young children are less likely to be held accountable for negative outcomes, particularly if it is perceived that they lack a well-developed ability to reason. In addition, a worker whose productivity has temporarily declined as a result of caring for his sick mother might receive feelings of sympathy as opposed to anger.

In sum, several scholars believe that beliefs about intentionality significantly influence moral judgments. For our purposes, intentionality has to do with actions that are instigated with a goal of producing specific outcomes and includes the actor's mental state. In terms of the relationship between intentionality, outcomes, and judgments; the most egregious transgressions are ones that result in negative outcomes, are under the control of the actor, and are not accompanied by mitigating circumstances. 


\section{Moral Judgment and Disagreement: A Cognitive Prototype Perspective}

Earlier, I reviewed five important theories relevant to the phenomenon of moral disagreement and identified their significant contributions to the field. I also provided a review of the empirical research suggests that there are several salient factors that influence moral judgments including one’s social context, claims for autonomy and consent, intentionality, and outcomes. In this section, I will argue that these factors are the primary properties of moral prototypes, and from a cognitive prototype perspective, I will develop a framework that will explain why some moral issues are more difficult to judge, prone to moral disagreement, and require additional cognitive resources.

To begin, it is important to note that within the scope of this project, the term moral judgment refers to the evaluation of interpersonal actions relevant to the moral domain. Moral judgments include perceptions of right and wrong, good or bad, and should not be confused with moral decision-making, which concerns the process of making a morally relevant decision as it is influenced by perceptions about what one should or ought to do.

It is conceivable that, over time, moral judgment developed as a mechanism to promote morally desirable actions and thwart morally undesirable actions. As discussed in earlier, actions relevant to the moral domain concern issues of justice, welfare, or rights (Turiel, 2002; Nucci,2001); and moral transgressions (i.e., breaches of justice, welfare, or rights) have a negative impact on spiritual, physical, or psychological wellbeing. As such, in a moral system, moral transgressions are likely to be condemned or punished for the purpose of reducing the frequencies of these occurrences. Conversely, actions that enhance spiritual, physical, or psychological well-being and improve the 
quality of life are likely to receive moral praise. In a moral system, praise encourages promoral behavior.

As a result of the interaction between humans and their social environment, moral knowledge is constructed over time and is the result of experience, training, and cognitive development (Kohlberg, 1969; Kohlberg et al., 1983; Nucci, 2001). Because it is easier to retrieve memory than to engage in reasoning processes, our brains have evolved to store information and conserve cognitive resources by developing automatic responses to particular stimuli (see Markman \& Gentner, 2001 for review). Training neuronal networks to recognize and quickly process frequently encountered stimuli makes these automatic responses possible. In the moral domain, automated moral judgments are likely to occur as the result of abstracting the properties of moral issues, constructing and refining moral prototypes, and encountering stimuli that closely resemble the prototype. Because the goal of moral judgment is to make an evaluation, it is plausible that within this context, particular features, such as intentionality, become more salient and relevant to making moral judgments.

In the moral domain, it is likely that two predominant moral prototypes are constructed: (a) the promoral prototype and (b) the immoral prototype, which will be discussed further shortly. Although the promoral prototype and immoral prototype probably share the same fundamental properties, these properties differ in their orientations. For instance, a prototypical immoral situation is one in which (a) the action, as it is interpreted ${ }^{8}$ in the social context, is not condoned (b) is intentionally malevolent, self-serving, or disregarding of others; (c) performed against the will of others; and (d) results in intentional, negative outcomes. 
In this view, the prototype structure is coherent as each property is directed towards an immoral orientation. In other words, when the social context does not condone the action and the action is driven by bad intentions, acted against the will of others, and results in intentional negative outcomes; the "morally bad" properties are consistent within the prototype. The "blame schema" research (e.g., Hermand, Mullet, Tomera, \& Touzart, 2001; Zelazo et al., 1996), which has found that adults often make judgments based on an additive or conjunctive rule (i.e., bad intentions + bad outcomes $=$ punish), supports this claim at a logical level. For example, when an agent intentionally and malevolently assaults a target (i.e., bad intention), we expect the target to experience negative outcomes such as physical harm and psychological distress (i.e., bad outcome). We do not expect the target to be happy as a result of the beating, nor do we expect the beating to enhance his quality of life in any way (i.e., result in a positive outcome); thus, a bad intention resulting in a positive outcome would be incoherent. This rule of consistency is also expected to hold true for the promoral prototype, which can be characterized as an action that is (a) considered virtuous within a social context; (b) intentionally benevolent and other-regarding; (c) consensual; and (d) results in intentional positive outcomes (see Figure 1). 


\section{Immoral Prototype}

Immoral Orientation

Promoral Orientation

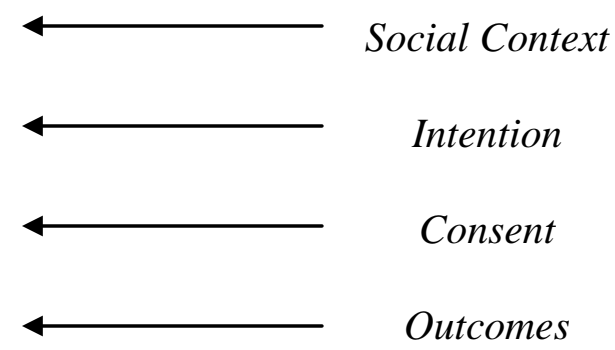

The properties of an immoral prototype are uniformly aligned towards and immoral orientation.

\section{Promoral Prototype}

Immoral Orientation

Promoral Orientation

Social Contex
Intention
Consent
Outcomes

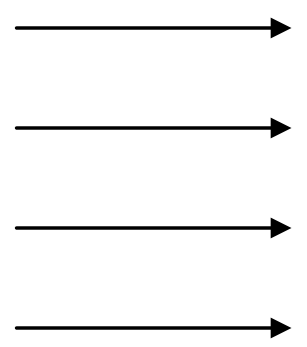

The properties of a promoral prototype are uniformly aligned towards a promoral orientation.

Figure 1. Property orientations of immoral and promoral prototypes. 
When the stimulus closely resembles either of these prototypes, it is predicted that moral judgment will be quick and automatic. However, when the attributes of the stimulus do not directly correlate with the properties of the prototype (i.e., when the stimulus deviates from the prototype), it is predicted that additional cognitive resources may be required to engage in the process of moral reasoning.

Reeder, Vonk, Ronk, Ham, and Lawrence (2004) conducted a study that supports this claim. One of the tasks in their study required the subjects to make judgments about a student's helping behavior. The subjects watched vignettes that depicted three conditions: (a) a no choice condition in which it was the student's job to perform the helping task; (b) a free choice condition in which the student offered to perform the helping task; and (c) an ulterior motives condition in which the subject was led to infer that the student performed the helping task for a personal gain. The investigators found that the subjects took significantly longer to make a judgment about the student in the ulterior motive condition. The authors speculate that the participants had to engage in more effortful processing as a result of the incoherence between the inferred, selfinterested intentions of the person and the positive outcome of her actions. In this example, the intentions and outcomes properties were at odds, producing a scenario of increasing complexity that deviates from the prototype.

As a result of the increasing complexity of nonprototypical issues, it is plausible that moral reasoning will be required to coordinate the properties and address inherent conflicts, such as assigning more weight to the most salient properties. Hence, situations that deviate from the prototype (e.g., situations that produce competing orientations or introduce additional mitigating circumstances like retaliation) may be more difficult to 
judge and effortful to process either within an individual or between persons. Due to the nature of nonprototypical issues, it is conceivable that as the complexity increases, so will the likelihood of producing heterogeneous responses (i.e., moral disagreement), particularly when none of the outcomes are appealing. In this view, nonprototypical issues will be more prone to moral disagreement because objective, measurable criteria may not be available for resolving the inherent conflicts; thus, choosing between the "lesser of two evils" might involve subjective preferences that weigh particular properties as more salient than others.

Not only does this theoretical framework predict that moral prototypes will lead to quicker judgments, but it also speculates that these prototypes provide us with the ability to make inferences about a given situation. Previous research suggests that people construct default knowledge structures to make quick predictions and judgments about others (see Karniol, 2003 for a review). When little information is available or cognitive resources are low, it is believed that the mind reverts to default representations about persons or situations. Thus, if moral prototypes are constructed from a set of fundamental properties, it is predicted that inferences about social meaning, intention, consent, and/or outcomes will be made when this information has not been made explicit.

For example, in the Turiel et al. study (1991), the participants were asked to make judgments about abortion. It was found that a number of positions changed after the participants were asked to consider abortion under specific circumstances such as in choosing the sex of the child or in cases of rape or incest. Because these responses shifted after intentionality was made explicit, we can deduce that the subjects made different intentionality inferences about abortion in its decontextualized form. 
In another study (Bersoff \& Miller, 1993), participants were asked to make a judgment about the following incident, “A man takes a book from a store without paying for it” (p. 676). Bersoff and Miller used this probe to represent a prototypical moral transgression and reported that nearly all participants categorized this probe in moral terms (i.e., as a theft). What is interesting about this probe is that neither the intentionality nor the consent orientations were made explicit; thus, it is likely that the participants relied on default representations to make inferences about these properties. For example, several rational, alternative interpretations are relevant to this probe including (a) the storeowner gave the man the book as a gift or (b) the man accidentally walked out with the book. It is feasible that if either of these conditions were made explicit, different responses would have been produced; therefore, if the participants interpreted this action to be a theft, the specific language of the probe probably led to particular inferences about intentionality and consent. As a result, it is possible that prototypes serve as default knowledge structures that influence moral judgment when little information is provided.

In order to develop a further understanding of the difference between moral prototypes and issues that deviate from the prototype, it is important to consider this model in context. The following example depicts a promoral prototype:

Sarah is a devoted advocate for children living in poverty. She believes that she has an obligation to do what she can to help people in need. Recently, she created a foundation that provides children in impoverished communities with free health care. The people in these communities value her contribution and are grateful for 
the help that it provides. Sarah's charity work helped to improve the health of the children she serves.

In this situation, the properties of the prototype are geared towards producing positive outcomes. In our social context, we support private foundations and individuals that seek to help children in need. The intentions of Sarah and her foundation are benevolent and other-regarding. The community consents to the foundation's activities, and there are positive outcomes in the form of improving the health and education of the children. It is predicted that such a scenario would garner moral praise, because it resembles the promoral prototype.

Conversely, the properties of an immoral prototype would be directed towards producing negative outcomes. An example of such a prototype is as follows:

Theresa is a single mother of three girls. Since her husband died, she has taken an interest in other men. She recently met a man with whom she wants to spend time. She doesn't care about her three kids and leaves them alone in the apartment to fend for themselves. Because Theresa is never home, the 8-year-old has become responsible for taking care of her younger sisters. There is no one else who can help with the children. The children are afraid to be alone and want their mother to stay home with them during the evenings. Theresa says that she cannot stay with them and continues to leave each night.

In this issue, each of the orientations is directed towards an immoral stance. First, there are two main social context concerns: (a) Theresa does not fulfill her obligation as a mother in which she has a duty to care for her children and (b) in our society, there is a belief that 8-year-olds are ill equipped to supervise children. Because these social 
context concerns are directed towards an immoral orientation, they take on an immoral stance. Additionally, Theresa is intentionally self-serving (bad intentionality); the children do not consent to their mother's actions (lack of consent); and there are actual emotional and potential physical consequences for leaving young children alone and unsupervised (negative outcomes). Because this scenario shares the same properties as a moral prototype with an immoral stance, it is predicted that most people would not condone Theresa's actions, and she would be subject to blame for the negative outcomes. In our next example, the orientation of intentionality has been changed and is directed towards producing positive outcomes. As a result, we now have an example of a scenario that deviates from the prototype as follows:

Theresa is a single mother of three girls. Since her husband died, Theresa has been unable to pay the bills. She recently found a second job, which has prevented the family from becoming homeless. Each evening she works a second shift. Because Theresa is rarely home, the 8-year-old has become responsible for taking care of her younger sisters. There is no one else who can help with the children. The children are afraid to be alone and want their mother to stay home with them during the evenings. Theresa says that she cannot stay with them and continues to leave each night.

The properties of this scenario take on incoherent stances (see Figure 2). 


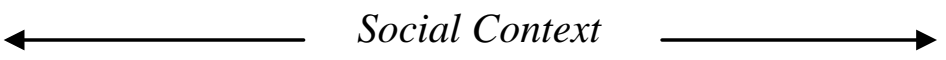

The mother has an obligation to promote the welfare of her children (good) vs. an 8-year-old lacks the skills and maturity required to supervise young children (bad).

\section{Intention}

Theresa accepts the job for the purpose of taking care of her children and promoting their welfare (good).

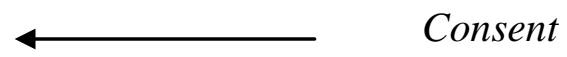

The children do not want their mother to leave each evening (bad).

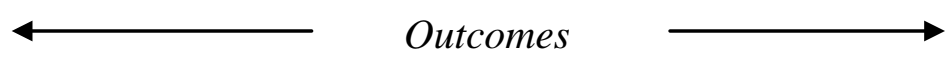

The family does not become homeless (good) vs. there is psychological distress and potential for physical harm (bad).

This nonprototype contains elements that are both morally good and morally bad.

Figure 2. Graphic display of a nonprototype example. 
This issue becomes more complex than the prototype, because several conflicts are created. First, there is a social context conflict. Even though Theresa is doing what she can to manage the welfare of her children, the 8-year-old is still too young to supervise her younger sisters by our standards. In addition, there is a conflict among the outcomes; preventing the family from becoming homeless does not eradicate the psychological distress and potential for physical harm that results from Theresa's prolonged absence. Finally, the intentions and consent properties are at odds as they are oriented towards opposing moral stances. In other words, the mother's intentions are good, but the children do not consent about being left alone. A nonprototypical scenario such as this might require one to use additional cognitive resources to weigh competing orientations in order to form a conclusion; thus, the increasing complexity of the issue makes it more difficult to judge than a moral prototype.

Sometimes, a nonprototypical issue can become extremely complex. Reverting back to the Terri Schiavo case, which was characterized as having a high level of moral disagreement, we can depict this public debate as having layers of incoherent properties. Not only were several of the orientations of the properties unclear and left to personal interpretation and speculation, such as not having a record of Terri's wishes (i.e., whether or not there was true consent), but the public was also divided in how they conceptualized persons in a vegetative state (i.e., a social context conflict). Even though Michael Schiavo stated that he took Terri off of life support in order to honor her wishes, there were broad speculations about whether or not this was his true intention (e.g., intentionality conflict). Finally, in the end, there were the competing potential outcomes of honoring a person's right to die vs. doing everything possible to save a life. 
It is important to note that in some cases people may believe that a situation is wrong even if there are no observable negative consequences. For instance, Haidt, Koller, and Dias (1993) found that people continued to see particular situations in moral terms even after the situation was rendered as harmless. Furthermore, the participants of the study often lacked the capacity to explain why. Haidt (2001) refers to this phenomenon as "moral dumbfounding” and claims that these moral responses provide evidence that moral judgments are more intuitive and less rational. In a description of his social intuitionist model, Haidt (2001) uses the following scenario as an example:

Julie and Mark are brother and sister. They are traveling together in France on summer vacation from college. One night they are staying alone in a cabin near the beach. They decide that it would be interesting and fun if they tried to make love. At the very least it would be a new experience for each of them. Julie was already taking birth control pill, but Mark uses a condom too, just to be safe. They both enjoy making love, but they decide not to do it again. They keep that night as their special secret, which makes them feel even closer to each other. (p.

According to Haidt, most of the participants who responded to this probe (though, importantly, not all) say that it was wrong for Mark and Julie to make love despite the scenario's design to strip away any potential for harm. Interestingly, in Haidt's attempt to control for harm, he inadvertently manipulated the properties of the moral issue. In this instance, the couple did not act out of ill intentions, the action was consensual, and there were no observable negative outcomes. Most of the subjects wanted to object to 
Mark’s and Julie’s actions but were unable to justify their responses (i.e., they were morally dumbfounded).

Haidt (2001) uses the phenomenon of moral dumbfounding to support his social intuitionist model of moral judgment. However, an alternative explanation is that particular concepts or actions, as they are constructed and understood within a social milieu, are loaded with moral meaning and cannot be completely dissociated from harm. This was made evident when Shweder et al., (1987) looked at how some actions, such as a widow wearing bright clothing, were considered to be morally wrong by the Brahmans and perceived as a social convention among Americans. For the Brahmans, this action, as it is conceptualized and made meaningful within their social context, is associated with negative outcomes in the form of producing a threat to the spiritual well-being of the deceased husband. In another example (Nucci, Camino, \& Sapiro, 1996), a significant number of children continued to view eating chicken with bare hands as a health risk and not merely as a social convention despite the investigators' efforts to minimize the possible threat of disease transfer by emphasizing the sanitary precautions of food handlers. The investigators explain that the children in the study lived in Northeastern Brazil in which there was a serious cholera epidemic. In this social context, eating chicken with bare hands was perceived to be a threat to their well-being, even after the probe suggested that it was safe.

Thus, inherent to some concepts (such as incest, rape, slavery, or oppression) is a representation of threat to well-being, which is intertwined into and inseparable from their constructed meanings. In other words, we cannot ask someone to imagine a benign form of "cancer," because the term cancer, as it is applied within our social context, is a 
disease that threatens life. Likewise, some of the participants in Haidt's study may not have been able to conceive of a benign form of incest, because the concept includes a notion of harm. To strip it of its associations with negative consequences is to change its meaning so that it no longer resembles its original concept. Thus, it is plausible that within a social context, some concepts convey a conceptual threat in which there is a perceived potential for harm inherent in the concept's constructed meaning. In other words, there is a belief that such a concept, when enacted, poses a threat to physical, psychological, and/or spiritual well-being and can affect the quality of life. As a result, even if the issue or action does not result in obvious negative outcomes, the potential for bad outcomes, as they are associated with particular concepts, could result in negative evaluations among people who have developed such notions. However, the social context property in itself is not the only salient factor in making moral judgments (as indicated by the fact that not every participant found Haidt's incest probe to be immoral). In some cases, people may weigh the intentions, consent, or outcomes properties more heavily. Thus, a social context conflict is not representative of a moral prototype. As with other nonprototypical issues, such a conflict is likely to produce moral disagreement as a result of its incoherent properties.

In order to depict the orientations of an issue with a conceptual threat consider the following:

The Johnson family is very wealthy and owns a chain of grocery stores. They live in a small town in which children are not lawfully required to attend school. In addition, there are no laws that prevent children from working full-time. The Johnson's 6-year-old son, William, told his parents that he wanted to work full- 
time in the family business. William's parents love him and believed that he would be happy and successful. They wanted to support him in any way that they could. His parents took him out of school, taught him how to run the business, and William managed the grocery stores until his retirement. He enjoyed every moment of his career and lived a very satisfying life. Was it wrong or all right for the Johnsons to allow William to quit school?

Such a scenario represents an issue that deviates from the moral prototype. In our society, we believe that children should go to school as means to access the culture's resources. Additionally, 6-year-olds are rarely given the authority to make important life decisions (such as whether or not they should stay in school), because it is believed that they lack the maturity and reasoning skills to make such important decisions. The parents, however, have benevolent intentions in supporting their son's wishes. Both the son and parents consent to such an action, and the action results in observable, positive outcomes. However, because the orientation of the social context property is at odds with the other properties, it is predicted that some people may have to engage in further processing to make a judgment. It is predicted that a situation like this would result in heterogeneous responses (i.e., moral disagreement).

In sum, the cognitive moral prototype model posits that, over time and as a result of cognitive development, training, and experience; people construct notions of morally blameworthy and morally praiseworthy behavior by abstracting out salient properties that lead to an ideal representation of each. These properties include (a) social context, (b) intentions, (c) claims for consent and autonomy, and (d) outcomes. The properties of immoral prototypes are coherently directed towards immoral orientations while the 
properties of promoral prototypes are uniformly directed towards promoral orientations. Nonprototypical issues deviate from the prototype and often represent a structure in which there are competing orientations or additional salient properties.

\section{Testing the Model}

Within this framework, I conducted two studies. The overarching goals for both experiments involved detecting the presence of moral prototypes. In theory, if people construct moral prototypes, then prototypical moral situations should lead to widespread agreement within a group. In other words, a very high percentage of people should judge prototypes as right or wrong, depending on the uniform promoral or immoral orientations of the properties. Because moral prototypes lack conflict or unusual circumstances, I predicted that these evaluations would be easy to judge and fall at the extreme ends of a right/wrong continuum.

My specific goals for the first study involved observing whether or not abstract moral transgressions would produce the same judgment effects as explicit, contextualized immoral prototypes. In other words, would the abstract cases activate default representations and act like moral prototypes? Furthermore, I wanted to test whether or not adding promoral properties to a moral transgression would produce additive effects and change judgments. If judgments moved from wrong to right as promoral properties were added, then it could be deduced that inferences about the properties are made when no explicit information is provided.

In the second study, I was specifically interested in the impact of promoral and immoral properties on judgment. My goal was to create nonprototypes by manipulating the orientations of the properties to produce systematic patterns of conflict within a set of 
scenarios. Then I compared the nonprototypes to the prototypes to see whether or not there would be a difference in judgments. I predicted that people would judge prototypes and nonprototypes differently. 


\section{CHAPTER III：STUDY 1}

\section{Method}

\section{Purpose}

The aim of this study was to determine whether or not the addition of promoral properties to an abstract moral transgression would cause shifts in moral judgment. It was predicted that adding explicit promoral intentions, consent, and outcome properties to an abstract transgression would gradually shift judgment responses from “wrong” to “right” along a six-point rating scale. It was also predicted that both the inferred properties of the abstract transgression and the explicit properties expressed in the contextualized immoral prototype would assume immoral stances; thus, the abstract transgression would activate a default immoral prototype that acts the same way as the contextualized prototype. If the abstract transgression and contextualized immoral prototype share the same structural properties but differ from nonprototypes (e.g., instances in which the scenarios share both promoral and immoral properties), then the following results would become evident:

1. The moral judgments of abstract transgressions and contextualized immoral prototypes will not differ significantly and the null hypothesis will not be rejected in this particular comparison.

2. The moral judgments of abstract transgressions and the contextualized immoral prototypes will differ significantly from the nonprototypes.

3. Abstract and contextualized immoral prototypes will result in a high percentage of agreement (i.e., most people will judge them as “wrong”). In comparison, the percentage of agreement will be lower among the nonprototype conditions, resulting 
in more heterogeneous judgments (i.e., disagreement in the form of producing judgements of both "right" and “wrong”).

4. As promoral intentions, consent, and outcome properties are explicitly added to abstract moral transgressions, they will accumulate weight. This weight will cause judgment ratings to shift from "wrong" to "right".

5. The language used to justify moral judgments will vary by condition.

\section{Participants}

The participants were recruited by a service that provides researchers and marketing specialists with subject pools consisting of adults (18 years and older) who are enrolled in a rewards program. Points are earned by responding to questionnaires and later redeemed for premiums (e.g., coupons or gift certificates). The participants are directed to the service via Internet ads and, once registered, receive links to a number of questionnaires or surveys from which they choose. They are given the opportunity to unsubscribe to the program or opt-out from any study at any time without consequences.

In this research study, the recruiting service provided potential participants with a link to my questionnaire. Before each volunteer was allowed to view and respond to it, he was asked to read and agree to an online consent form. If a person chose not to consent to the research, the web site redirected him to an exit page.

In order to achieve a power of .80 and to detect a .25 effect size (in standard deviation units) with an alpha of .05, I estimated that approximately 200 participants would be required for this study (i.e., 40 participants per group for a total of five groups). In total, 242 people responded to the questionnaire. Six participants were dropped for providing “ 1 's" across all quantitative responses and for providing unusable responses for 
each qualitative section, such as “I don’t care,” “\#\#\#\#\#\#, or “duhhh”. Three participants were dropped for reporting that they are under the age of 18 . In order to control for cultural and developmental variables, the participants were limited to adults (18 years or older), who were born and are currently living in the United States. Of the 233 remaining participants $($ Mage $=40, M d n=39$, mode $=22$, range $=18-71, S D=14 ;$ Mincome $=$ $\$ 60,591, M d n=45,000$, mode $=25,000$, range $=0-650,000, S D=64,803), 105$ (45.1\%) are male and 128 (54.9\%) are female. Self-reported ethnic/racial identities included the following: 39 (16.7\%) Asian, 59 (25.3\%) Black or African American, 49 (21\%) Hispanic or Latino, 85 (36.5\%) White or Caucasian, and 1 (.4\%) Native American. The highest level of education included 90 (38.6\%) participants who completed high school, 64 (27.5\%) who completed an associates or vocational program, 55 (23.6\%) who completed a bachelors program, and 24 (10.3\%) who completed a graduate degree.

\section{Measures and Procedures}

I presented each group of participants with four hypothetical scenarios and asked them to make judgments of right or wrong using a six-point, bipolar scale ( 1 = very wrong $-6=$ very right). The neutral category (i.e., "neither agree nor disagree”) was omitted in order to require participants to make a judgment and reduce the effects of over-relying on the neutral category (Linacre, 2004; Stone, 2004).

In addition, I asked the participants to explain their justifications in a short written response. Initially, a 12-category coding scheme was developed to analyze this data. However, it failed to produce a strong reliability measure. In order to improve reliability, two separate schemes were constructed. Coding Scheme 1 was created to focus on the core attributes of this study and consists of four categories: intentions, consent, outcomes, 
and no properties (see Table I for more detailed descriptions and examples). With the assistance of a graduate student, 20 percent of the responses were coded for reliability. Coding Scheme 1 obtained an interrater agreement level of .92 (Cohen’s Kappa).

TABLE I

DEFINITIONS AND SAMPLES OF JUSTIFICATION CATEGORIES

\begin{tabular}{|c|c|c|}
\hline Category & Definition & Sample Responses \\
\hline \multirow[t]{3}{*}{ Intentions } & $\begin{array}{l}\text { Judgements based on the perceived intentions of } \\
\text { the actor. }\end{array}$ & $\begin{array}{l}\text { "He didn’t want to see anyone getting } \\
\text { hurt." }\end{array}$ \\
\hline & & $\begin{array}{l}\text { "He had good intentions because he } \\
\text { wanted to 'save' other's lives." }\end{array}$ \\
\hline & & "She tried to correct the situation." \\
\hline \multirow[t]{3}{*}{ Consent } & Judgments influenced by whether or not the act & "Sarah wanted to die." \\
\hline & whether or not the target of the action was a & "He had permission.” \\
\hline & & "She agreed to it." \\
\hline \multirow[t]{3}{*}{ Outcomes } & $\begin{array}{l}\text { Judgments that focus on the actual, inferred, or } \\
\text { predicted outcomes of the action. }\end{array}$ & "It could paralyze them." \\
\hline & & "She lived." \\
\hline & & "He could get sued.” \\
\hline \multirow[t]{3}{*}{ No Property } & $\begin{array}{l}\text { Judgements that do not contain any language } \\
\text { about moral properties. }\end{array}$ & “They were wrong.” \\
\hline & & "The ten commandments." \\
\hline & & "None." \\
\hline
\end{tabular}


Due to the large number of "no property" responses, a second coding scheme was developed by using categories from previous research on moral judgment (Brehl, 2008; Nucci \& Turiel, 1993). The aim of this coding scheme was to find out how participants justified their moral judgments when they did not use language about intentions, consent, or outcomes. After running Coding Scheme 1 across all responses, the no property cases were then selected and subject to Coding Scheme 2, which included the following categories: authority and norms, principled justifications, contingent justifications, counterfactuals, undefined responses, and uncodable responses (see Table II for detailed descriptions and examples). A graduate student and I coded 20 percent of the "no property” responses for reliability and obtained an interrater agreement level of .88 (Cohen's Kappa). When there was disagreement, we talked about the response until we reached an agreement about how to categorize it. 
TABLE II

DEFINITIONS AND SAMPLES OF JUSTIFICATION CATEGORIES FOR THE NO PROPERTIES CASES

\begin{tabular}{|c|c|c|}
\hline Category & Definition & Sample Responses \\
\hline $\begin{array}{l}\text { Authority and } \\
\text { Norms }\end{array}$ & $\begin{array}{l}\text { The judgments involve statements about God's } \\
\text { law, religious authority, secular rules and laws, or } \\
\text { consensus. }\end{array}$ & $\begin{array}{l}\text { “The ten commandments.” } \\
\text { “That's why we have laws.” } \\
\text { "It is a crime.” }\end{array}$ \\
\hline Principled & $\begin{array}{l}\text { The judgments involve perceptions of justice, } \\
\text { fairness, rights, and/or concern for the welfare of } \\
\text { others. }\end{array}$ & $\begin{array}{l}\text { "Treat others the way that you want to } \\
\text { be treated." } \\
\text { "We have an obligation to help others } \\
\text { in need." } \\
\text { "If it doesn't belong to you, you } \\
\text { should not touch it." }\end{array}$ \\
\hline Counterfactual & $\begin{array}{l}\text { Judgments that state that the actor(s) should have } \\
\text { chosen a more desirable or alternative course of } \\
\text { action. }\end{array}$ & $\begin{array}{l}\text { "She should have asked for help." } \\
\text { "He should have disconnected them } \\
\text { from the hinges, so they could be } \\
\text { rehung." } \\
\text { "Performing the Heimlich maneuver } \\
\text { would have been a better choice." }\end{array}$ \\
\hline Contingent & $\begin{array}{l}\text { The judgments are based on the perception that } \\
\text { more information is needed to make a judgment } \\
\text { or that additional, unstated circumstances exist. }\end{array}$ & $\begin{array}{l}\text { "I would have liked to pick both right } \\
\text { and wrong, because it depends on the } \\
\text { situation." } \\
\text { "There are times when it is okay to } \\
\text { kill a person." } \\
\text { "Stealing is wrong unless it is a life or } \\
\text { death situation." }\end{array}$ \\
\hline Undefined & $\begin{array}{l}\text { The judgments are unelaborated statements of } \\
\text { right or wrong. }\end{array}$ & $\begin{array}{l}\text { "It’s immoral." } \\
\text { "Because it is the right thing to do." } \\
\text { "Stealing is not good." }\end{array}$ \\
\hline Uncodable & $\begin{array}{l}\text { The statement cannot be used or coded for the } \\
\text { purposes of this study. }\end{array}$ & $\begin{array}{l}\text { "None" } \\
\text { "I don’t know why you are asking me } \\
\text { this." } \\
\text { "N/A" }\end{array}$ \\
\hline
\end{tabular}


Per participant, each written justification was coded as a single response. If more than one category was present in a response, it was partitioned into proportional values. For example, if a response contained language relevant to consent, outcomes, and intentions, each of those categories received a value of .33. This same process was used for Coding Scheme 2.

The participants were randomly (via computer generation) assigned to groups according to treatment condition. As mentioned in the previous chapter, the social context property acts like a moral prescription in which the beliefs, knowledge, and values of a person lead to statements of what one "should" or "ought” to do. Previous research (Nucci, 2001; Shweder et al., 1987) suggests that most people, across social contexts, think that one should not kill, steal, damage personal property, or hit others and would judge these actions as morally wrong. One reason why most people judge these actions as wrong may be that, within the social context, people construct moral meaning over time and make inferences about the properties of these actions; thus, when the actions are discussed in the abstract, they activate immoral prototypes. For example, when a person thinks about killing in the abstract, it is likely that the person assumes that the killing is intentionally malevolent, against the will of the target, and results in a tragic outcome even though the nature of these properties are not explicitly stated. In order to test this notion, the themes of killing, stealing, property destruction, and hitting were used to construct the abstract moral transgression items in which the intentions, consent, and outcomes properties were purposefully unstated under this condition (see Appendix for the full question set). In the abstract transgression condition, the participants in Group 1 were asked to use the six-point rating scale to complete a sentence for each of the four 
themes. For example, the property destruction sentence stated, "It is to destroy another person's property.”

Using the same themes of killing, hitting, stealing, and property destruction, Group 2's condition introduced a contextualized, hypothetical scenario, which included explicit information about promoral intentions. For example, building on the property destruction example, the participants had to read the following scenario:

One day, while his neighbors were on vacation, Bob noticed that the shutters on his neighbors' home were dangerously close to falling off their hinges. Because the shutters were located above a busy walkway, he feared that they would fall and hurt someone. Bob used a sledgehammer to destroy and remove the shutters. In this situation, Bob has good intentions but destroys someone else’s property. No additional information about promoral consent or outcomes was provided. At the end of each scenario in conditions 2 - 5, the participants were asked to complete the same basic sentence using the six-point "wrong” vs. "right” rating scale. The sentence is as follows: It was __ for [name] to [do the action]. In this property destruction example, the participants had to respond to the statement: It was for Bob to destroy his neighbors' shutters.

The scenarios that I presented to Groups 3 and 4 followed the same pattern in which promoral consent and outcomes variables were added to the promoral intentions. In Group 3’s scenarios, promoral intentions and consent were explicitly stated as in the example provided below:

One day, while his neighbors were on vacation, Bob noticed that the shutters on his neighbors' home were dangerously close to falling off their hinges. Because 
the shutters were located above a busy walkway, he feared that they would fall and hurt someone. Bob called his neighbors to explain the situation, and they gave him permission to remove and dispose of the shutters. Bob used a sledgehammer to destroy and remove the shutters.

In this case, the neighbors consent to Bob's actions; thus, the consent property assumed a promoral stance.

Group 4 responded to scenarios designed to include promoral intentions, consent, and outcomes as exemplified in the scenario below:

One day, while his neighbors were on vacation, Bob noticed that the shutters on his neighbors' home were dangerously close to falling off their hinges. Because the shutters were located above a busy walkway, he feared that they would fall and hurt someone. Bob called his neighbors to explain the situation, and they gave him permission to remove and dispose of the shutters. Bob used a sledgehammer to destroy and remove the shutters. Thanks to Bob, no one was hurt.

In this scenario, explicit language is used to tell the reader that Bob is responsible for preventing injuries; thus, a promoral outcome is added. As the promoral properties are added to each condition, if the moral judgments progressively shift further towards the "very right" end of the rating scale across the first four conditions, I could deduce that the accumulation of these promoral properties produced additive effects.

Group 5 responded to a set of scenarios that followed a different pattern. The properties in these scenarios were made explicit and uniformly directed towards an 
immoral stance, representing a contextualized immoral prototype as depicted in the example below:

One day, Bob’s neighbors went away on vacation. Bob hates his neighbors and enjoys seeing them upset. In the middle of the night, Bob used a sledgehammer to destroy and remove the shutters on his neighbor's home. After the neighbors returned, they were shocked to find that their home had been vandalized. All of the shutters were destroyed. They were heartbroken and had to pay a lot of money to replace them.

If the abstract transgression activates a default prototype in which the inferred orientation of the moral properties are uniformly oriented towards an immoral stance, then the responses should not differ significantly from the explicit, contextualized scenarios in which the properties are oriented in the same direction. In other words, if the judgment ratings of the abstract and contextualized prototypes do not significantly differ, then the results would suggest that they might be functionally equivalent to each other and, perhaps, share the same property orientations.

\section{Results}

\section{Data Analysis Plan and Data Reduction}

Moral Judgment Ratings. This study compared the effects of the five treatment conditions on moral judgment. Although the moral judgment dependent variables of all four scenarios could have been used in a multivariate design, it was not within the framework of this study to make those types of comparisons (e.g., whether or not killing is judged as more wrong than stealing). Thus, instead of running a doubly multivariate analysis, which has less power and is more difficult to interpret, a decision was made to 
perform four, one-way ANOVA's - one for each moral transgression (i.e., killing, hitting, stealing, and the destruction of personal property) as depicted by Figure 3.

\begin{tabular}{|l|l|l|l|l|}
\hline \multicolumn{1}{|c|}{ Group 1 } & \multicolumn{1}{c|}{ Group 2 } & \multicolumn{1}{c|}{ Group 3 } & \multicolumn{1}{c|}{ Group 4 } & \multicolumn{1}{c|}{ Group 5 } \\
$\begin{array}{l}\text { abstract } \\
\text { transgression with } \\
\text { no explicit } \\
\text { information about } \\
\text { intentions, consent, } \\
\text { or outcomes }\end{array}$ & $\begin{array}{l}\text { added explicit } \\
\text { promoral intention }\end{array}$ & $\begin{array}{l}\text { added explicit } \\
\text { promoral intention } \\
\text { + consent }\end{array}$ & $\begin{array}{l}\text { added explicit } \\
\text { promoral intention } \\
\text { + consent + } \\
\text { outcomes }\end{array}$ & $\begin{array}{l}\text { Contextualized, } \\
\text { immoral prototype } \\
\text { with explicitly } \\
\text { stated immoral } \\
\text { properties }\end{array}$ \\
\hline
\end{tabular}

Figure 3. Structure of ANOVA research design by groups.

Exploratory data analyses revealed that the scenarios are separate constructs; thus, none of them were omitted or collapsed into a group. In addition, applying transformation algorithms to the dependent variable and the removal of outliers did not improve the skewed curve of the distribution. Therefore, the untransformed data was used for this study and the outliers were maintained for the benefit of improving interpretation and increasing power, respectively. In order to control for Type 1 error across the multiple tests, a Bonferroni adjustment was made and the alpha was set at .01 (two-tailed). Preliminary ANCOVA analyses were performed on each scenario set to test whether or not there were significant demographic related interactions or main effects on moral judgment ratings. The following independent variables were tested: type of condition, race, sex, and education with age and income acting as the covariates. Main effects and interactions of the demographic variables were not significant, $F=.207-$ 
2.337, $p \geq .02, n s$. In addition, the covariates were not significant, $F=.015-1.193, p \geq$ $.27, n s$. As a result, the demographic variables were dropped from subsequent analyses. Moral Judgment Frequencies and Percentages. In addition to the four, one way ANOVA's, the frequencies and percentages of right and wrong responses of each scenario across all five conditions were noted. The purpose of including these descriptive statistics is to observe the percentage of moral agreement. Because the properties of prototypes are aligned towards a uniform promoral or immoral stance, it was predicted that they would produce a high percentage of moral agreement and result in homogeneous responses (e.g, 100\% of the judgments indicate that the situation was wrong). However, since nonprototypes deviate from the prototype, this framework also proposes that they would act as a source of moral disagreement, thus, yielding a greater percentage of heterogeneous responses. If a participant rated the scenario as "very wrong”, "wrong”, or "somewhat more wrong than right", the judgment was coded as $1=$ "wrong”. If the participant rated the scenario as "very right", "right”, or "somewhat more right than wrong", then the judgment was coded as $2=$ "right". Then, the total number and percentages of "wrong” or "right" responses were computed. A high percentage of either "wrong” or "right” responses indicates agreement (e.g., 100\% of the responses were coded as "wrong”), while more proportionally divided responses indicate disagreement (e.g., 30\% responded “right” and 70\% as “wrong”).

Moral Justifications. The aim of this analysis was to determine whether or not the type of condition has an effect on moral justifications. For instance, will the promoral intentions scenarios in Condition 2 produce more responses that include concerns about 
intentions? To test this question, the participants were asked to provide a written explanation of their moral judgments after they rated each scenario.

As a result of the between-groups design, proportional data, and cells with values of 0 , a decision was made to run an ANOVA. In order to analyze the data categorized by Coding Scheme 1, a 5 condition (abstract transgressions, intentions, consent, outcomes, contextualized prototype) x 4 moral justification (no property, intentions, consent, and outcomes) mixed model, repeated measures ANOVA was run separately on each of the four scenarios (i.e., hitting, stealing, property destruction, and killing), with the justification codes serving as the repeated measure.

Since the framework of this proposed model of moral judgment compares moral prototypes with nonprototypes, hypothesis testing was not conducted on the data generated from Coding Scheme 2. Instead, I consider this data to be supplemental information for the reader. Thus, only the frequencies and percentages will be used to report the results of Coding Scheme 2 (see results in Table III). The categories that result in 20 percent of the responses or greater will be noted along with the ANOVA results. 
TABLE III

SUBCATEGORIES OF JUSTIFICATIONS CODED AS “NO PROPERTY” ACTUAL NUMBER (\% OF TREATMENT GROUP) [\% OF TOTAL] PER SCENARIO

\begin{tabular}{|c|c|c|c|c|c|c|}
\hline \multirow[b]{2}{*}{$\begin{array}{c}\text { Moral } \\
\text { Justifications }\end{array}$} & \multicolumn{6}{|c|}{ Type of Treatment } \\
\hline & $\begin{array}{l}\text { Abstract } \\
\text { Prototype }\end{array}$ & Intentionality & Consent & Outcomes & $\begin{array}{c}\text { Contextualized } \\
\text { Prototype }\end{array}$ & Total \\
\hline \multicolumn{7}{|l|}{ Hitting } \\
\hline $\begin{array}{l}\text { Authority and } \\
\text { Norms }\end{array}$ & 1 (3) $[1]$ & 0 & 0 & 0 & 7 (24) [06] & 8 [ 7] \\
\hline Principle & 12.5 (37) [11] & $1(5)[1]$ & $4(25)[3]$ & $7(43)[6]$ & 4 (14) [04] & $28.5[25]$ \\
\hline Counterfactual & $2.5(7)[2]$ & $11(55)[10]$ & $6(38)[5]$ & $4(25)[3]$ & 5 (17) [04] & $28.5[25]$ \\
\hline Contingency & 6 (18) [ 5] & $3(15)[3]$ & 0 & $1(6)[1]$ & 1 ( 3$)[01]$ & $11[10]$ \\
\hline Undefined & 10 (29) [ 9] & $3(15)[3]$ & 3 (19) [ 3] & $2(13)[2]$ & $8(28)[07]$ & 26 \\
\hline Uncodable & 2 ( 6) [ 2] & $2(10)[2]$ & $3(19)[3]$ & $2(13)[2]$ & 4 (14) [03] & 13 [11] \\
\hline Total & $34 \quad[30]$ & [17] & [14] & [14] & [25] & $\mathrm{N}=115$ \\
\hline \multicolumn{7}{|l|}{ Property Damage } \\
\hline $\begin{array}{l}\text { Authority and } \\
\text { Norms }\end{array}$ & 6 (14) [ 4] & 0 & 0 & 0 & $6(18)[4]$ & 12 [ 9] \\
\hline Principle & 19 (45) [14] & $9.5(34)[7]$ & $3(20)[2]$ & $5(26)[4]$ & $10(30)[7]$ & $46.5[34]$ \\
\hline Counterfactual & 1 ( 2) [ 1] & $6.5(23)[5]$ & 7 (47) [ 5] & $5(26)[4]$ & 0 & 19.5 [14] \\
\hline Contingency & 2 (5) [ 1] & 0 & $1(6)[1]$ & $1(5)[1]$ & 0 & $4 \quad[3]$ \\
\hline Undefined & 11 (26) [ 8] & 11 (39) [ 8] & $2(3)[1]$ & $4(21)[3]$ & $14(42)[10]$ & $42[31]$ \\
\hline Uncodable & 3 ( 7) [ 2] & 1 (4) [ 1] & 2 (13) [ 1] & $4(21)[3]$ & 3 ( 9) [ 2] & 13 [ 9] \\
\hline Total & {$[31]$} & [20] & [11] & [14] & [24] & $\mathrm{N}=137$ \\
\hline \multicolumn{7}{|l|}{ Stealing } \\
\hline $\begin{array}{l}\text { Authority and } \\
\text { Norms }\end{array}$ & $8(21)[4]$ & 5 (15) [ 3] & $5(13)[3]$ & $2.5(7)[1]$ & $10(23)[5]$ & $30.5[16]$ \\
\hline Principle & $12(32)[6]$ & 3 ( 9$)[2]$ & $3(8)[2]$ & $5.5(16)[3]$ & 5 (12) [ 3] & 28.5 [15] \\
\hline Counterfactual & $2(5)[1]$ & 12 (36) [ 6] & $16(41)[9]$ & 12 (35) [ 6] & $2(4)[1]$ & $44 \quad[24]$ \\
\hline Contingency & $1(3)[1]$ & 0 & 0 & 0 & 0 & $1[1]$ \\
\hline Undefined & $14(34)$ [ 7] & 12 (36) 6] & $13(33)[7]$ & $12(35)[6]$ & $25(58)[13]$ & 76 \\
\hline Uncodable & $1(3)[1]$ & 1 ( 3) [ 1] & $2(5)[1]$ & 2 (6) [ 1] & 1 ( 2) [ 1] & $\begin{array}{ll}7 & {[4]}\end{array}$ \\
\hline Total & [ 20] & [18] & [21] & [18] & [23] & $\mathrm{N}=187$ \\
\hline \multicolumn{7}{|l|}{ Killing } \\
\hline $\begin{array}{l}\text { Authority and } \\
\text { Norms }\end{array}$ & 14 (34) [ 9] & $4(16)[3]$ & 16 (53) [10] & 8 (33) [ 5] & $11(33)[7]$ & 53 [35] \\
\hline Principle & 7 (17) [ 5] & $3(12)[2]$ & $3(10)[2]$ & $5(21)$ [ 3] & $9(27)[6]$ & 27 [18] \\
\hline Counterfactual & $2(5)[1]$ & $5(20)[4]$ & $1(3)[1]$ & $1(4)[1]$ & $2(6)[1]$ & $11[7]$ \\
\hline Contingency & 7 (17) [ 5] & $2(8)[1]$ & $1(3)[1]$ & 0 & 0 & $10[7]$ \\
\hline Undefined & $11(27)[$ ] $]$ & $7(28)[5]$ & $7(23)[5]$ & $9(38) \quad[6]$ & $7(21)$ [ 5] & 41 [27] \\
\hline Uncodable & 0 & $4(16)[3]$ & $2(7)[1]$ & $1(4)[1]$ & $4(12)[3]$ & 11 [ 7] \\
\hline Total & [27] & [16] & [20] & [16] & [21] & $N=153$ \\
\hline
\end{tabular}

Note. Due to rounding, percentages may not add up to 100. 
Because the sphericity and normality assumptions were violated in each analysis, the Huynh-Felt statistic will be reported in lieu of the omnibus F, because it makes an adjustment based on the degree of failed sphericity (Tabachnick \& Fidell, 2001). In order to protect against the increased probability of Type 1 error that results from multiple testing, a Bonferroni adjustment was made to produce a more conservative alpha, and the tests will be considered significant at the .01 (two-tailed) level. Finally, transformations of the data did not yield different conclusions from the untransformed data, so the results are reported using the untransformed data for the purpose of improving interpretation.

\section{Moral Judgment Ratings}

In all four ANOVAs, the Levene's test indicated that the assumption of homogeneity of variances was violated: property destruction $F(4,228)=31.97, p<$ .001 ; hitting $F(4,228)=8.90, p<.001$; killing $F(4,228)=63.18, p<.001$; and stealing $F(4,228)=13.75, p<.001$. Thus, the Welch statistic was used in lieu of the omnibus Fratio. In each analysis, the Welch test showed statistically significant differences among the five levels of moral properties: property destruction $F(4,104.56)=66.65, p<.001$, $\eta^{2}=.52 ;$ hitting $F(4,108.40)=100.14, p<.001, \eta^{2}=.64 ;$ killing $F(4,102.96)=39.01$, $p<.001, \eta^{2}=0.37$; and stealing $F(4,107.56)=12.73, p<.001, \eta^{2}=.15$. Thus, in each ANOVA, the null hypothesis was rejected, signifying that at least one group’s mean differed from the others (see Table IV for a summary of statistics by group). Post-hoc comparisons were conducted using the Games-Howell test, which is a test that is often performed when the population variances are not equal. 
TABLE IV

MORAL JUDGMENT RATINGS BY CONDITION AND SCENARIO

MEAN (SD) [95\% CI]

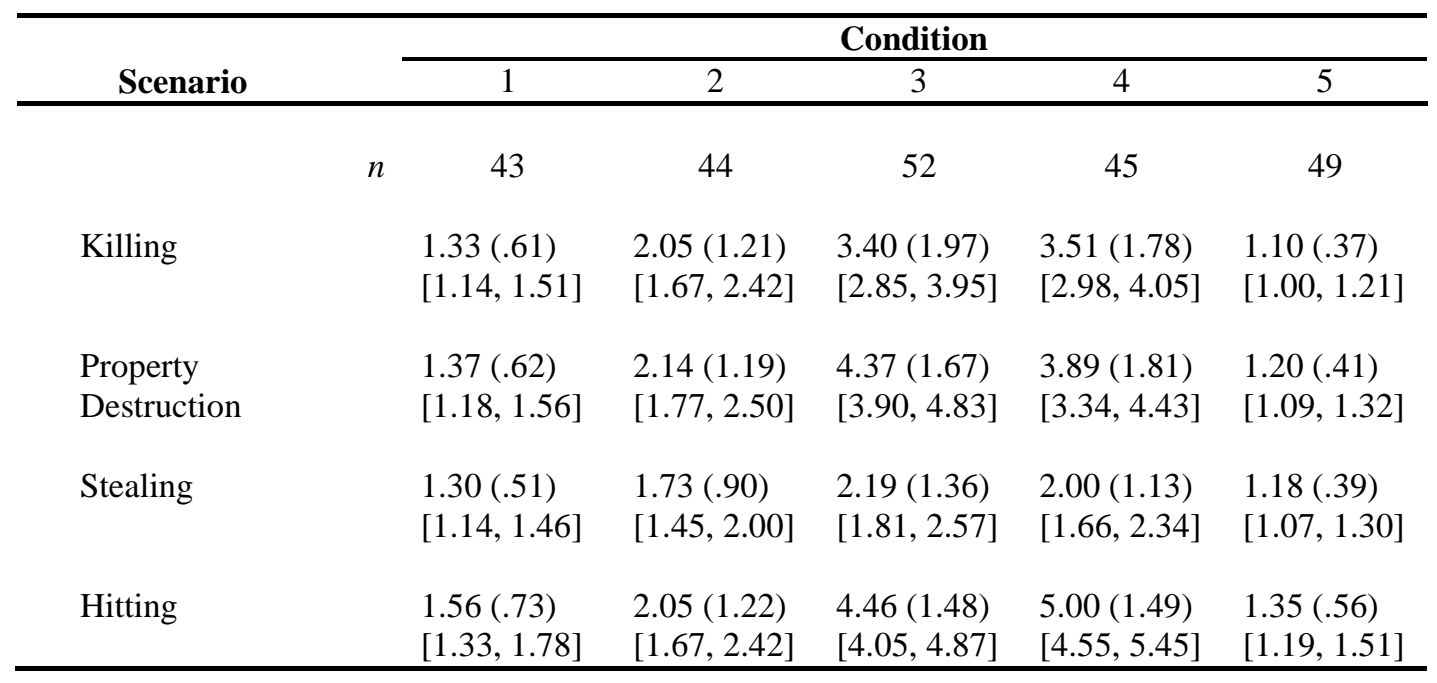

Note. Moral judgment ratings are based on a 6-point, bi-polar scale ( 1 = very wrong, 6 = very right).

Participants were randomly assigned to the following treatment groups:

$$
\begin{aligned}
& 1=\text { abstract immoral prototype condition } \\
& 2=\text { promoral intentionality condition } \\
& 3=\text { promoral intentionality and consent condition } \\
& 4=\text { promoral intentionality, consent, and outcomes condition } \\
& 5=\text { contextualized, immoral prototype }
\end{aligned}
$$


Property Destruction. The Games-Howell test revealed that the abstract moral transgressions condition was rated significantly more wrong than the intentions $p=.003$, $d=-.81,99 \%$ CI [-1.45, -.07], consent $p<.001, d=-2.38$, CI [-3.84, -2.15$]$ and and outcomes $p<.001, d=-1.86$, CI [-3.50, -1.54$]$ conditions. The intentions condition was rated as significantly more wrong than the consent $p<0.001, d=-1.53$, CI [-3.21, -1.25$]$ and outcomes $p<.001, d=-1.14$, CI [-2.85, -.66] conditions. The contextualized immoral prototypes were significantly more wrong than the intentions, $p<0.001, d=$ 1.05, CI [-1.58, -.28], consent $p<.001, d=-2.61$, CI [-3.98, -2.35], and outcomes $p<$ $.001, d=-2.05$, CI $[-3.64,-1.73]$ conditions. As predicted, there were no significant differences between the abstract transgression and contextualized immoral prototypes $p=$ $.56, d=0.32$, CI [-0.21, 0.54], ns.

Hitting. The abstract moral transgression condition was rated as more wrong than the consent $p<.001, d=-2.49$, CI [-3.69, -2.12], and outcomes $p<.001, d=-2.92$, CI [-4.29, -2.60] conditions. The intentions condition was rated as more wrong than the consent $p<0.001, d=-1.78$, CI [-3.34, -1.49] and outcomes $p<.001, d=-2.17$, CI [3.92, -1.98] conditions. The contextualized immoral prototype was rated as more wrong than the intentions $p=.008, \mathrm{~d}=-0.74$, CI $[-1.38,-0.01]$, consent $p<.001, d=-2.79$, CI [-3.86, -2.37], and outcomes $p<.001, \mathrm{~d}=-3.24$, CI [-4.46, -2.84$]$ conditions. As predicted, there were no significant differences between the abstract transgression and contextualized immoral prototype conditions $p=.543, d=0.32$, CI [-0.25, 0.68], ns.

Killing. The abstract moral transgression was rated as more wrong than the intentions $p=.007, d=-0.75$, CI [-1.42, -0.02], consent $p<.001, d=-1.42$, CI [-3.06, 1.10], and outcome $p<.001, d=-1.64$, CI [-3.15, -1.22] conditions. The intentions 
scenario was rated as more wrong than consent $p=0.001, d=-0.82$, CI $[-2.47,-0.25]$ and outcomes $p<.001, d=-0.96$, CI [-2.55, -0.38$]$ conditions. The contextualized immoral prototype was rated as more wrong than the intentions, $p<0.001, d=-1.06$, CI [-1.60, 0.29], consent $p<.001, d=-1.62$, CI [-3.26, -1.35], and outcomes $p<.001, d=-1.88$, CI [-3.34, -1.48] conditions. As predicted, there were no significant differences between the abstract transgression and contextualized immoral prototype conditions $p=.231, d=$ 0.46, CI [-0.14, 0.58], ns.

Stealing. The abstract transgression was rated as more wrong than the consent $p<$ $.001, d=-0.87$, CI [-1.58, -0.20] and outcomes $p=.003, d=-0.80$, CI [-1.33, -0.07$]$ conditions. It approached a statistically significant difference with the intentions condition $p=.06, d=-0.59$, CI [-0.95, 0.11$]$, ns. The contextualized prototype was rated as more wrong than the intentions $p=.004, d=-0.79$, CI [-1.04, -0.04$]$, consent, $p<.001$, $d=-1.01$, CI [-1.68, -0.34$]$, and outcomes $p<.001, d=-0.97$, CI $[-1.42,-0.21]$ conditions. As predicted, there were no significant difference between the abstract transgression and contextualized immoral prototype conditions $p=.732, d=0.26$, CI [-0.95, 0.11], ns.

Overall, the findings from this data analysis support the hypothesis that the abstract transgressions and the contextualized prototypes produce similar judgments. All of the contextualized immoral prototypes were viewed as significantly more wrong than the nonprototypes, while the abstract moral transgressions were viewed significantly more wrong than most of the nonprototypes. These findings also suggest that promoral properties can change judgment ratings and may produce additive effects when combined. 


\section{Moral Judgment Frequencies and Percentages}

As predicted, the abstract transgression and contextualized prototype conditions resulted in perfect or near perfect agreement (98\% - 100\%) that the action was wrong. In the abstract and contextualized prototype conditions (Conditions 1 and 5), all 92 (100\%) participants who responded to the killing, property destruction, and stealing scenarios rated these scenarios as wrong. In the hitting scenario, 42 out of 43 (98\%) participants rated the abstract transgression as wrong while all 49 (100\%) participants rated the contextualized prototype scenario as wrong. The remaining conditions (Conditions 2 4), in which promoral properties were added to each scenario, resulted in greater

disagreement (i.e., the responses included a mix of "right” and "wrong” judgments). Under these conditions, the percentage of participants that rated the nonprototype scenarios as wrong ranges from 13\% - 96\%. (See Table V for a listing of sample sizes, frequencies, and percentages.) 
TABLE V

FREQUENCY (AND PERCENTAGE) OF WRONG AND RIGHT RESPONSES

\begin{tabular}{|c|c|c|c|c|c|c|c|c|c|c|c|}
\hline \multirow[b]{2}{*}{ Scenario } & & \multicolumn{10}{|c|}{ Condition } \\
\hline & & \multicolumn{2}{|c|}{1} & \multicolumn{2}{|c|}{2} & \multicolumn{2}{|c|}{3} & \multicolumn{2}{|c|}{4} & \multicolumn{2}{|c|}{5} \\
\hline & \multirow[t]{2}{*}{$n$} & \multicolumn{2}{|c|}{43} & \multicolumn{2}{|c|}{44} & \multicolumn{2}{|c|}{52} & \multicolumn{2}{|c|}{45} & \multicolumn{2}{|c|}{49} \\
\hline & & W & $\mathrm{R}$ & W & $\mathrm{R}$ & $\mathrm{W}$ & $\mathrm{R}$ & W & $\mathrm{R}$ & W & $\mathrm{R}$ \\
\hline Killing & & $\begin{array}{c}43 \\
(100)\end{array}$ & $\begin{array}{c}0 \\
(0)\end{array}$ & $\begin{array}{c}38 \\
(86)\end{array}$ & $\begin{array}{c}6 \\
(14)\end{array}$ & $\begin{array}{c}25 \\
(48)\end{array}$ & $\begin{array}{c}27 \\
(52)\end{array}$ & $\begin{array}{c}19 \\
(42)\end{array}$ & $\begin{array}{c}26 \\
(58)\end{array}$ & $\begin{array}{c}49 \\
(100)\end{array}$ & $\begin{array}{c}0 \\
(0)\end{array}$ \\
\hline $\begin{array}{l}\text { Property } \\
\text { Destruction }\end{array}$ & & $\begin{array}{c}43 \\
(100)\end{array}$ & $\begin{array}{c}0 \\
(0)\end{array}$ & $\begin{array}{c}37 \\
(84)\end{array}$ & $\begin{array}{c}7 \\
(16)\end{array}$ & $\begin{array}{c}15 \\
(29)\end{array}$ & $\begin{array}{c}37 \\
(71)\end{array}$ & $\begin{array}{c}16 \\
(29)\end{array}$ & $\begin{array}{c}29 \\
(64)\end{array}$ & $\begin{array}{c}49 \\
(100)\end{array}$ & $\begin{array}{c}0 \\
(0)\end{array}$ \\
\hline Stealing & & $\begin{array}{c}43 \\
(100)\end{array}$ & $\begin{array}{c}0 \\
(0)\end{array}$ & $\begin{array}{c}42 \\
(96)\end{array}$ & $\begin{array}{c}2 \\
(5)\end{array}$ & $\begin{array}{c}41 \\
(79)\end{array}$ & $\begin{array}{c}11 \\
(21)\end{array}$ & $\begin{array}{c}41 \\
(91)\end{array}$ & $\begin{array}{c}4 \\
(9)\end{array}$ & $\begin{array}{c}49 \\
(100)\end{array}$ & $\begin{array}{c}0 \\
(0)\end{array}$ \\
\hline Hitting & & $\begin{array}{c}42 \\
(98)\end{array}$ & $\begin{array}{c}1 \\
(2)\end{array}$ & $\begin{array}{c}38 \\
(86)\end{array}$ & $\begin{array}{c}6 \\
(14)\end{array}$ & $\begin{array}{c}12 \\
(23)\end{array}$ & $\begin{array}{c}40 \\
(77)\end{array}$ & $\begin{array}{c}6 \\
(13)\end{array}$ & $\begin{array}{c}39 \\
(87)\end{array}$ & $\begin{array}{c}49 \\
(100)\end{array}$ & $\begin{array}{c}0 \\
(0)\end{array}$ \\
\hline
\end{tabular}

Note. Due to rounding, the percentages may not add up to 100 . The 6-pt. moral judgment rating scale $(1=$ very wrong -6 = very right) was collapsed into a 2-pt. scale $(1-3=$ wrong, $4-6=$ right $)$. $\mathrm{W}=$ wrong; $\mathrm{R}=$ right; 1 = abstract transgression condition; 2 = promoral intentionality condition; 3 = promoral intentionality and consent condition; 4 = promoral intentionality, consent, and outcomes condition; and 5 = contextualized, immoral prototype. 


\section{Moral Justifications}

As predicted, there appears to be a relationship between type of condition and justification.

Hitting. Using a criterion of $\alpha=.01$ (two-tailed), type of condition did not produce a significant main effect. However, moral justifications led to a significant within-subjects main effect, Huynh-Feldt $F(2.24,511.27)=46.69, \mathrm{p}<.001, \eta_{\mathrm{p}}{ }^{2}=.17$. Holding type of condition constant, pairwise comparisons (Bonferroni) on the four levels of moral justifications indicated that most of the participants' responses fell into the no property category (see Table VI for significance tests results and a listing of proportional means, standard deviations, and confidence intervals). Thus, the no property justification was used significantly more often than the outcome, intentions, and consent justifications. When participants did use moral properties as justifications for their responses, outcomes and intentions justifications were used significantly more often than consent. Among the 115 no property cases, the highest percentages of responses were categorized as principle (28.5\%), counterfactuals (28.5\%), and undefined (26\%) justifications. 


\section{TABLE VI}

PAIRWISE COMPARISONS (BONFERRONI) OF

MORAL JUSTIFICATIONS IN THE HITTING SCENARIO

\begin{tabular}{|c|c|c|c|c|}
\hline & \multicolumn{4}{|c|}{ Moral Justifications } \\
\hline & No Property & Intentions & Consent & Outcomes \\
\hline $\begin{array}{l}\text { Mean }(S D) \\
{[95 \% \mathrm{CI}]}\end{array}$ & $\begin{array}{c}.51(.50) \\
{[0.44,0.57]}\end{array}$ & $\begin{array}{c}.21(.41) \\
{[0.16, .26]}\end{array}$ & $\begin{array}{c}.05(.20) \\
{[0.02,0.07]}\end{array}$ & $\begin{array}{c}.24(.41) \\
{[0.19,0.29]}\end{array}$ \\
\hline $\begin{array}{l}\text { Moral } \\
\text { Justifications } \\
\text { No Property }\end{array}$ & & & & \\
\hline $\begin{array}{r}p \\
\text { Cohen's } d \\
95 \% \text { CI }\end{array}$ & --- & $\begin{array}{c}<.001^{*} \\
0.66 \\
{[0.17,0.43]}\end{array}$ & $\begin{array}{c}<.001^{*} \\
1.21 \\
{[0.37,0.56]}\end{array}$ & $\begin{array}{c}<.001^{*} \\
0.59 \\
{[0.14,0.40]}\end{array}$ \\
\hline $\begin{array}{r}\text { Intentions } \\
\mathrm{p} \\
\text { Cohen's } d \\
\mathrm{CI}\end{array}$ & --- & --- & $\begin{array}{c}<.001^{*} \\
0.50 \\
{[0.08,0.24]}\end{array}$ & $\begin{array}{c}1.00 \\
-0.07 \\
{[-0.13,0.07]}\end{array}$ \\
\hline $\begin{array}{r}\text { Consent } \\
p \\
\text { Cohen's } d \\
\text { CI }\end{array}$ & --- & --- & --- & $\begin{array}{c}<.001^{*} \\
-0.59 \\
{[-0.27,-0.12]}\end{array}$ \\
\hline $\begin{array}{r}\text { Outcomes } \\
\mathrm{p} \\
\text { Cohen's } d \\
\mathrm{CI}\end{array}$ & --- & --- & --- & --- \\
\hline
\end{tabular}

Note. *The mean difference is significant at the .01 level. 
The overall moral justification main effect was qualified by a significant interaction between condition and moral justification, Huynh-Feldt $F(8.97,511.27)=$ 8.90, $p<.001 ; \eta_{\mathrm{p}}{ }^{2}=.14$. This interaction suggests that the type of condition had a bearing on the participants’ moral justifications.

Abstract transgression condition. When participants were presented with the abstract transgression condition, pairwise comparisons (Bonferroni) revealed that the no properties justification appeared significantly more often than the outcomes $p<.001, d=$ 1.73, 95\% CI [.35, .95], intentions $p<.001, d=2.50$, CI [.55, .99], and consent $p<.001$, $d=2.94$, CI $[.65, .98]$ justifications. (Refer to Table VII for a listing of proportional means, standard deviations, and confidence intervals by cell for all four types of scenarios.) Of the moral properties, the outcome justification had the greatest proportional mean, but it only approached statistical significance when it was compared to consent $p=.028, d=0.63$, CI $[.01, .31]$, n.s. Thus, when participants responded to the abstract transgression condition, they most often relied on justifications that did not include moral prototype language. Among the 34 no property responses in this condition, the participants tended to use principle (37\%) and undefined (29\%) justifications. 
TABLE VII

PROPORTIONAL MEANS (SD) [95\% CI] BY CELL FOR EACH SCENARIO

\begin{tabular}{|c|c|c|c|c|c|}
\hline \multirow[b]{2}{*}{$\begin{array}{c}\text { Moral } \\
\text { Justifications }\end{array}$} & \multicolumn{5}{|c|}{ Condition } \\
\hline & $\begin{array}{c}\text { Abstract } \\
\text { Transgression } \\
n=43\end{array}$ & $\begin{array}{c}\text { Intentions } \\
n=44\end{array}$ & $\begin{array}{c}\text { Consent } \\
n=52\end{array}$ & $\begin{array}{c}\text { Outcomes } \\
n=45\end{array}$ & $\begin{array}{c}\text { Contextualized } \\
\text { Prototype } \\
n=49\end{array}$ \\
\hline \multicolumn{6}{|l|}{ Hitting } \\
\hline No Property & $\begin{array}{c}.81(.39) \\
{[0.69 .0 .94]}\end{array}$ & $\begin{array}{c}.45(.50) \\
{[0.30,0.61]}\end{array}$ & $\begin{array}{c}.31(.47) \\
{[0.18 \quad 0.44]}\end{array}$ & $\begin{array}{c}.37(.49) \\
{[0.23 .0 .53]}\end{array}$ & $\begin{array}{c}.59(.50) \\
{[0.45,0.73]}\end{array}$ \\
\hline Intentions & $\begin{array}{c}.05(.18) \\
{[-0.01,0.10]}\end{array}$ & $\begin{array}{c}.36(.48) \\
{[0.22,0.51]}\end{array}$ & $\begin{array}{c}.27(.44) \\
{[0.15,0.39]}\end{array}$ & $\begin{array}{c}.02(.15) \\
{[-0.02,0.07]}\end{array}$ & $\begin{array}{c}.35(.48) \\
{[0.21,0.49]}\end{array}$ \\
\hline Consent & $\begin{array}{c}.00(.00) \\
{[0.00,0.00]}\end{array}$ & $\begin{array}{c}.00(.00) \\
{[0.00,0.00]}\end{array}$ & $\begin{array}{c}.15(.35) \\
{[0.06,0.25]}\end{array}$ & $\begin{array}{c}.06(.16) \\
{[0.01,0.10]}\end{array}$ & $\begin{array}{c}.02(.14) \\
{[-0.02,0.06]}\end{array}$ \\
\hline Outcomes & $\begin{array}{c}.16(.36) \\
{[0.05,0.27]}\end{array}$ & $\begin{array}{c}.18(.39) \\
{[0.06,0.30]}\end{array}$ & $\begin{array}{c}.27(.43) \\
{[0.18,0.44]}\end{array}$ & $\begin{array}{c}.54(.47) \\
{[0.40,0.69]}\end{array}$ & $\begin{array}{c}.04(.20) \\
{[-0.02,0.10]}\end{array}$ \\
\hline \multicolumn{6}{|l|}{ Property } \\
\hline No Property & $\begin{array}{c}.98(.15) \\
{[0.85,1.11]}\end{array}$ & $\begin{array}{c}.64(.49) \\
{[0.51,0.77]}\end{array}$ & $\begin{array}{c}.29(.46) \\
{[0.17,0.41]}\end{array}$ & $\begin{array}{c}.42(.50) \\
{[0.29,0.55]}\end{array}$ & $\begin{array}{c}.67(.47) \\
{[0.55,0.80]}\end{array}$ \\
\hline Intentions & $\begin{array}{c}.00(.00) \\
{[-0.08,0.08]}\end{array}$ & $\begin{array}{c}.07(.20) \\
{[-0.01,0.14]}\end{array}$ & $\begin{array}{c}.07(.22) \\
{[0.00,0.14]}\end{array}$ & $\begin{array}{c}.12(.26) \\
{[0.05,0.20]}\end{array}$ & $\begin{array}{c}.21(.40) \\
{[0.14,0.29]}\end{array}$ \\
\hline Consent & $\begin{array}{c}.00(.00) \\
{[-0.10,0.10]}\end{array}$ & $\begin{array}{c}.18(.37) \\
{[0.02,0.28]}\end{array}$ & $\begin{array}{c}.59(.47) \\
{[0.50,0.68]}\end{array}$ & $\begin{array}{c}.29(.43) \\
{[0.19,0.39]}\end{array}$ & $\begin{array}{c}.00(.00) \\
{[-0.09,0.09]}\end{array}$ \\
\hline Outcomes & $\begin{array}{c}.02(.15) \\
{[-0.06,0.10]}\end{array}$ & $\begin{array}{c}.11(.30) \\
{[0.04,0.19]}\end{array}$ & $\begin{array}{c}.06(.21) \\
{[-0.02,0.13]}\end{array}$ & $\begin{array}{c}.14(.33) \\
{[0.07,0.22]}\end{array}$ & $\begin{array}{c}.11(.29) \\
{[0.04,0.19]}\end{array}$ \\
\hline \multicolumn{6}{|l|}{ Stealing } \\
\hline No Property & $\begin{array}{c}.88(.32) \\
{[0.76,1.00]}\end{array}$ & $\begin{array}{c}.75(.44) \\
{[0.63,0.87]}\end{array}$ & $\begin{array}{c}.75(.44) \\
{[0.64,0.86]}\end{array}$ & $\begin{array}{c}.76(.43) \\
{[0.64,0.87]}\end{array}$ & $\begin{array}{c}.88(.33) \\
{[0.77,1.00]}\end{array}$ \\
\hline Intentions & $\begin{array}{c}.00(.00) \\
{[-0.09, .09]}\end{array}$ & $\begin{array}{c}.10(.30) \\
{[0.02,0.19]}\end{array}$ & $\begin{array}{c}.13(.33) \\
{[0.05,0.21]}\end{array}$ & $\begin{array}{c}.20(.40) \\
{[0.11,0.29]}\end{array}$ & $\begin{array}{c}.07(.25) \\
{[-0.01,0.15]}\end{array}$ \\
\hline Consent & $\begin{array}{c}.00(.00) \\
{[-0.02,0.02]}\end{array}$ & $\begin{array}{c}.00(.00) \\
{[-0.02,0.02]}\end{array}$ & $\begin{array}{c}.03(.15) \\
{[0.01,0.04]}\end{array}$ & $\begin{array}{c}.00(.00) \\
{[-0.02,0.02]}\end{array}$ & $\begin{array}{c}.00(.00) \\
{[-0.02,0.02]}\end{array}$ \\
\hline Outcomes & $\begin{array}{c}.12(.32) \\
{[0.03,0.20]}\end{array}$ & $\begin{array}{c}.15(.35) \\
{[0.07,0.23]}\end{array}$ & $\begin{array}{c}.09(.28) \\
{[0.02,0.17]}\end{array}$ & $\begin{array}{c}.04(.21) \\
{[-0.04,0.13]}\end{array}$ & $\begin{array}{c}.05(.21) \\
{[-0.03,0.13]}\end{array}$ \\
\hline \multicolumn{6}{|l|}{ Killing } \\
\hline No Property & $\begin{array}{c}.98(.15) \\
{[0.84,1.11]}\end{array}$ & $\begin{array}{c}.59(.50) \\
{[0.46,0.73]}\end{array}$ & $\begin{array}{c}.65(.48) \\
{[0.53,0.78]}\end{array}$ & $\begin{array}{c}.53(.50) \\
{[0.40,0.67]}\end{array}$ & $\begin{array}{c}.59(.50) \\
{[0.47,0.72]}\end{array}$ \\
\hline Intentions & $\begin{array}{c}.00(.00) \\
{[-0.05,0.05]}\end{array}$ & $\begin{array}{c}.05(.21) \\
{[0.00,0.09]}\end{array}$ & $\begin{array}{c}.00(.00) \\
{[-0.04,0.04]}\end{array}$ & $\begin{array}{c}.02(.15) \\
{[-0.02,0.07]}\end{array}$ & $\begin{array}{c}.05(.21) \\
{[0.01,0.09]}\end{array}$ \\
\hline Consent & $\begin{array}{c}.00(.00) \\
{[-0.12,0.12]}\end{array}$ & $\begin{array}{c}.31(.46) \\
{[0.17,0.45]}\end{array}$ & $\begin{array}{c}.36(.48) \\
{[0.22,0.49]}\end{array}$ & $\begin{array}{c}.33(.46) \\
{[0.22,0.45]}\end{array}$ & $\begin{array}{c}.16(.36) \\
{[0.05,0.27]}\end{array}$ \\
\hline Outcomes & $\begin{array}{c}.02(.15) \\
{[-0.06,0.11]}\end{array}$ & $\begin{array}{c}.08(.26) \\
{[0.00,0.16]}\end{array}$ & $\begin{array}{c}.07(.24) \\
{[-0.01,0.14]}\end{array}$ & $\begin{array}{c}.11(.30) \\
{[0.03,0.19]}\end{array}$ & $\begin{array}{c}.17(.38) \\
{[0.10,0.25]}\end{array}$ \\
\hline
\end{tabular}


Intentions condition. When promoral intentions language was introduced in the scenario, the intentions, outcomes, and no property justifications did not significantly differ from each other. However, the participants used the intentions and no property justifications significantly more often than consent $p<.001, d=1.06$, CI $[.16, .57] ; p$ $<.001, d=1.27, \mathrm{CI}[.25, .67]$, and the use of outcomes as a justification approached statistical significance when compared to consent $p=.021, d=0.65$, CI [.02, .34], n.s. Therefore, when the condition included a promoral intention for hitting, the participants most often justified their responses by using language relevant to the no properties, intentions, or outcomes categories. Among the 20 no properties responses, the participants tended to rely on counterfactual (55\%) justifications.

Consent condition. When promoral consent language was added to the scenario, there were no statistical differences between any of the moral justifications (for all comparisons, $p>$.59); thus, participants did not rely on one justification category more than any other. Among the 16 no properties responses, the participants often produced counterfactual (38\%) justifications.

Outcomes condition. When an explicit promoral outcome was added to the scenarios in the fourth condition, the outcomes justification had the greatest proportional mean and was used significantly more often than the consent $p<.001, d=1.37$, CI [.28, ,70] and intentions $p<.001, d=1.49$, CI [.31, .74] justifications. Likewise, the no properties justification was also used significantly more often than the consent $p=.002, d$ $=0.85$, CI $[.09, .55]$ and intentions $p<.001, d=0.97, \mathrm{CI}[.13, .57]$ justifications. As a result, there was no significant difference between the no properties and outcomes justifications, and the participants most often included these justifications in their 
responses. Among 16 no properties justifications, there was a higher percentage of principle (43\%) justifications.

Contextualized prototype condition. In the immoral contextualized prototype condition, in which the intentions, consent, and outcomes properties were explicitly directed towards immoral stances, the no properties justification had the greatest proportional mean and was used more often than the consent $p<.001, d=1.55$, CI [.36, .78] and outcomes $p<.001, d=1.44$, CI [.32, .78] justifications. Of the three moral properties, intentions received the greatest proportional mean and did not differ significantly from the no properties justification. Like the no properties category, the intentions justification was used significantly more often than the consent $p<.001, d=$ 0.93, CI $[.12, .53]$ and outcomes $p=.002, d=0.84$, CI [.09, .52] justifications. Thus, the participants tended to use the intentions and no properties justifications most often under this condition. Among the 29 no properties justifications in this condition, the undefined (28\%) and authority and norms (24\%) categories received the highest percentages of responses.

Property Destruction. Using a criterion of $\alpha=.01$ (two-tailed), type of condition did not produce a significant main effect. However, moral justifications led to a significant within-subjects main effect, Huynh-Feldt $F(2.35,535.11)=91.44, \mathrm{p}<.001$, $\eta_{\mathrm{p}}{ }^{2}=.29$. Holding type of condition constant, pairwise comparisons (Bonferroni) on the four levels of moral justifications indicated that most of the participants' responses fell into the no property category. (See Table VIII for significance tests results and a listing of proportional means, standard deviations, and confidence intervals.) Thus, the no property justification was used significantly more often than the outcome, intentions, and consent 
justifications. When participants did use moral properties as justifications for their responses, consent justifications were used significantly more often than intentions and outcomes. Of the 137 no properties cases, the highest percentage of responses were principle (46.5\%) and undefined (42\%) justifications.

\section{TABLE VIII}

PAIRWISE COMPARISONS (BONFERRONI) OF MORAL JUSTIFICATIONS IN THE PROPERTY DESTRUCTION SCENARIO

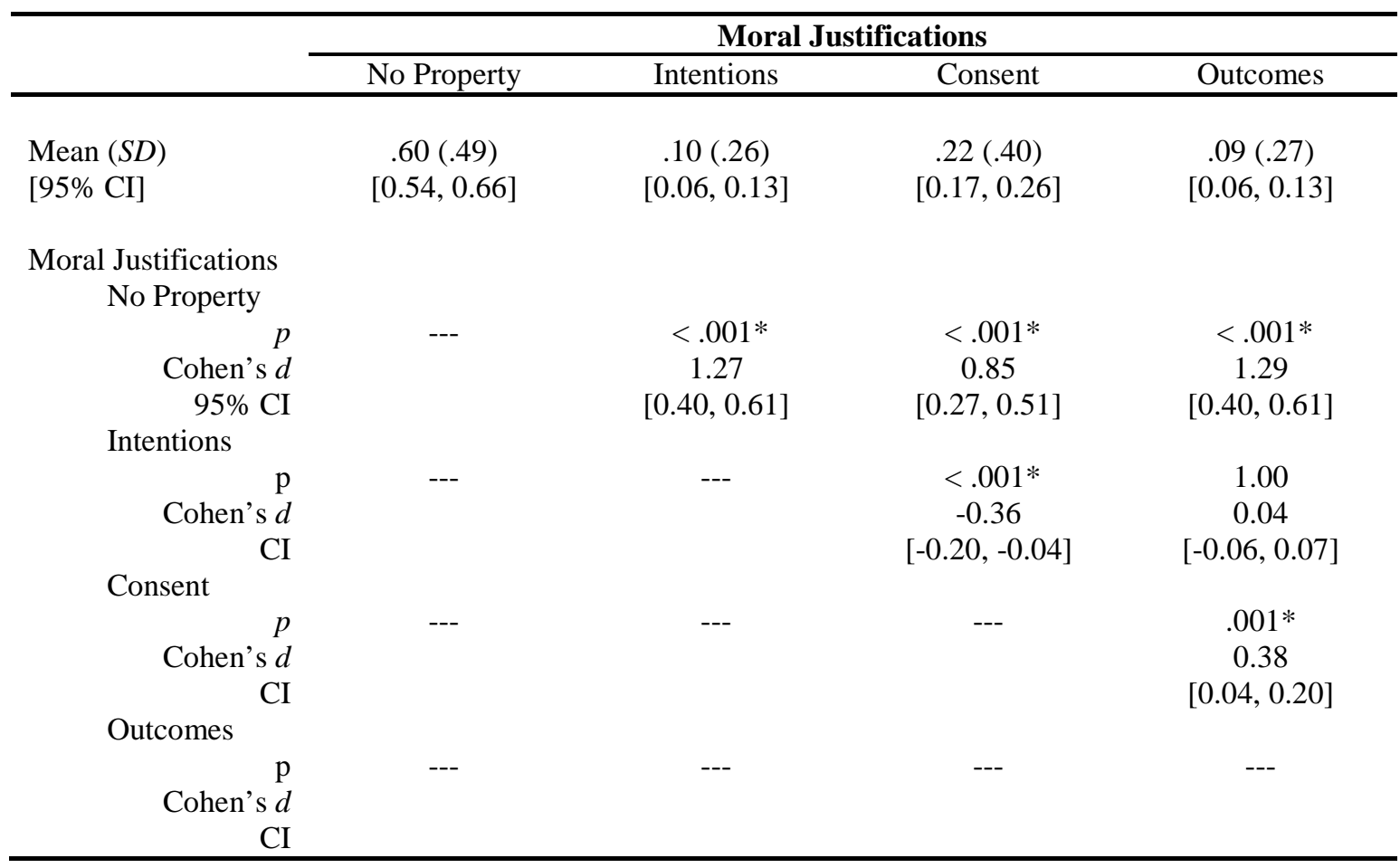

Note. *The mean difference is significant at the .01 level. 
The overall moral justification main effect was qualified by a significant interaction between condition and moral justification, Huynh-Feldt $F(9.39,535.11)=$ 14.90, $\mathrm{p}<.001 ; \eta_{\mathrm{p}}{ }^{2}=.21$. This interaction suggests that the type of condition had a bearing on the participants’ moral justifications.

Abstract transgression condition. Pairwise comparisons revealed that the no properties justification appeared significantly more often than the outcomes $p<.001, d=$ 6.40, 95\% CI [0.83, 1.08], intentions $p<.001, d=9.24$, CI [0.91, 1.04], and consent $p$ $<.001, d=9.24$, CI $[0.91,1.04]$ justifications. Thus, when participants responded to the abstract transgression condition, they most often relied on justifications that did not include moral properties language. Of the 42 no properties cases, the highest percentages of responses were the principled (45\%) and undefined (26\%) justifications.

Intentions condition. When promoral intentions language was introduced in the scenario, the participants used the no property justification significantly more often than consent $p=.002, d=1.06$, CI $[0.13,0.78]$; outcomes $p<.001, d=1.30$, CI $[0.24,0.81]$, and intentions $p<.001, d=1.52$, CI [0.32, 0.82]. Therefore, when the condition included a promoral intention for causing property destruction, the participants most often did not use moral properties language. Of the 28 "no properties” cases, the highest percentage of responses were the undefined (39\%), principle (34\%), and counterfactual (23\%) justifications.

Consent condition. When promoral consent language was introduced in the scenario, the more frequent use of the no property justification approached statistical significance when compared to the intentions $p=.03, d=0.61, \mathrm{CI}[0.01,0.43]$, n.s. and outcomes justifications $p=.02, d=0.64$, CI[0.03, 0.44], n.s. However, the most 
frequently used justification was consent, which was used significantly more often than the intentions $p<.001, d=1.42$, CI $[0.30,0.74]$ and outcomes $p<.001, d=1.46$, CI[0.31, 0.74] justifications. Thus, the participants' responses tended to include language concerned about consent or explanations that did not include moral property language. Of the 15 no property justifications, the highest percentage of responses fell into the counterfactual (47\%) category.

Outcomes condition. When promoral outcomes language was introduced in the scenario, the more frequent use of the no property justification approached statistical significance when compared to the outcomes $p=.06, d=0.66$, CI[-0.01, 0.56], n.s. and intentions justifications $p=.02, d=0.75$, CI[0.03, 0.57], n.s. Of the 19 no property justifications, the highest percentage of responses were counterfactual (26\%), principle (26\%) undefined (21\%), and uncodable (21\%) justifications.

Contextualized prototype condition. In the contextualized prototype condition in which the intentions, consent, and outcomes properties were explicitly directed towards immoral stances in the scenario, the no properties justification had the greatest proportional mean and was used more often than the intentions $p<.002, d=1.05$, CI $[0.14,0.78]$, outcomes $p<.001, d=1.43$, CI [0.29, 0.83], and consent $p<.001, d=2.02$, CI $[0.49,0.86]$ justifications. Of the three moral properties, intentions received the greatest proportional mean and was used significantly more often than the consent $p=$ $.002, d=0.74$, CI [0.06, 0.37]. The use of the outcomes justification approached statistical significance when compared to the consent justification $p=.06, d=0.54$, CI[$0.00,0.23]$, n.s. Of the 33 no properties cases, the highest percentage of responses were the undefined (42\%) and principle (30\%) justifications. 
Stealing. Using a criterion of $\alpha=.01$ (two-tailed), type of condition did not produce a significant main effect. However, moral justifications led to a significant within-subjects main effect, Huynh-Feldt $F(1.75,399.32)=294.88, \mathrm{p}<.001, \eta_{\mathrm{p}}{ }^{2}=.56$. Holding type of condition constant, pairwise comparisons (Bonferroni) on the four levels of moral justifications indicated that most of the participants' responses fell into the no property category (see Table IX for significance tests results and a listing of proportional means, standard deviations, and confidence intervals). Thus, the no property justification was used significantly more often than the outcome, intentions, and consent justifications. When participants did use moral properties as justifications for their responses, intentions and outcomes justifications were used significantly more often than consent, but outcomes and intentions did not differ significantly from each other. Of the no property cases, undefined (41\%) and counterfactual (24\%) justifications were used in the highest percentage of responses. 
TABLE IX

PAIRWISE COMPARISONS (BONFERRONI) OF MORAL JUSTIFICATIONS

IN THE STEALING SCENARIO

\begin{tabular}{|c|c|c|c|c|}
\hline & \multicolumn{4}{|c|}{ Moral Justifications } \\
\hline & No Property & Intentions & Consent & Outcomes \\
\hline $\begin{array}{l}\text { Mean }(S D) \\
{[95 \% \mathrm{CI}]}\end{array}$ & $\begin{array}{c}.80(.40) \\
{[0.75,0.86]}\end{array}$ & $\begin{array}{c}.10(.30) \\
{[0.06,0.14]}\end{array}$ & $\begin{array}{c}.01(.07) \\
{[0.00,0.01]}\end{array}$ & $\begin{array}{c}.09(.28) \\
{[0.05,0.13]}\end{array}$ \\
\hline $\begin{array}{c}\text { Moral Justifications } \\
\text { No Property }\end{array}$ & & & & \\
\hline $\begin{array}{r}p \\
\text { Cohen's } d \\
95 \% \text { CI }\end{array}$ & --- & $\begin{array}{c}<.001^{*} \\
1.98 \\
{[0.59,0.81]}\end{array}$ & $\begin{array}{c}<.001^{*} \\
2.75 \\
{[0.73,0.87]}\end{array}$ & $\begin{array}{c}<.001^{*} \\
2.06 \\
{[0.61,0.82]}\end{array}$ \\
\hline $\begin{array}{r}\text { Intentions } \\
\text { Cohen's } d \\
\mathrm{CI}\end{array}$ & --- & --- & $\begin{array}{c}<.001^{*} \\
0.41 \\
{[0.04,0.15]}\end{array}$ & $\begin{array}{c}1.00 \\
0.03 \\
{[-0.06,0.08]}\end{array}$ \\
\hline $\begin{array}{l}\text { Consent } \\
\qquad \begin{array}{r}p \\
\text { Cohen’s } d \\
\text { CI }\end{array}\end{array}$ & --- & --- & --- & $\begin{array}{c}<.001 * \\
-0.39 \\
{[-0.14,-0.04]}\end{array}$ \\
\hline $\begin{array}{r}\text { Outcomes } \\
\text { Cohen's } d \\
\mathrm{CI}\end{array}$ & --- & --- & --- & --- \\
\hline
\end{tabular}

Note. ${ }^{*}$ The mean difference is significant at the .01 level.

There was no significant interaction between condition and moral justification, Huyn-Feldt $F(7.00,399.324)=1.74, p=.10, \eta_{\mathrm{p}}{ }^{2}=.03$, n.s.; therefore, no further pairwise comparisons were made.

Abstract transgression condition. Among the 38 no properties cases under the abstract transgression condition, the participants tended to rely on undefined (34\%), principle (32\%), and authority and norms (21\%) justifications. 
Intentions condition. Among the 33 no properties responses under the promoral intentions condition, the participants often used counterfactual (36\%) and undefined (36\%) justifications.

Consent condition. Among the 39 no properties cases under the condition in which promoral consent was added, the participants often used counterfactual (41\%) and undefined (33\%) justifications.

Outcomes condition. When a promoral outcome was added to the condition, among the 34 no property cases, counterfactual (41\%) and undefined (33\%) justifications were often used.

Contextualized prototype condition. Under the contextualized prototype condition, among the 43 no properties cases, most of the responses fell into the undefined (58\%) and authority and norms categories.

Killing. Using a criterion of $\alpha=.01$ (two-tailed), type of condition did not produce a significant main effect. However, moral justifications led to a significant within-subjects main effect, Huynh-Feldt $F(1.93,440.60)=128.17, \mathrm{p}<.001, \eta_{\mathrm{p}}{ }^{2}=.36$. Holding type of condition constant, pairwise comparisons (Bonferroni) on the four levels of moral justifications indicated that most of the participants' responses fell into the no property category (see Table $\mathrm{X}$ for significance tests results and a listing of proportional means, standard deviations, and confidence intervals). Thus, the no property justification was used significantly more often than the intentions, consent, and outcomes justifications. When participants did use moral properties as justifications for their responses, consent justifications were used significantly more often than intentions and outcomes justifications, and the outcomes justification was used significantly more often 
than the intentions justification. Of the 153 no properties cases, authority and norms (35\%) and undefined (27\%) produced the greatest percentage of responses.

TABLE X

PAIRWISE COMPARISONS (BONFERRONI) OF MORAL JUSTIFICATIONS IN THE KILLING SCENARIO

\begin{tabular}{|c|c|c|c|c|}
\hline & \multicolumn{4}{|c|}{ Moral Justifications } \\
\hline & No Property & Intentions & Consent & Outcomes \\
\hline $\begin{array}{l}\text { Mean }(S D) \\
{[95 \% \mathrm{CI}]}\end{array}$ & $\begin{array}{c}.67(.47) \\
{[0.61,0.73]}\end{array}$ & $\begin{array}{c}.02(.15) \\
{[0.01,0.04]}\end{array}$ & $\begin{array}{c}.24(.42) \\
{[0.18,0.28]}\end{array}$ & $\begin{array}{c}.09(.28) \\
{[0.06,0.13]}\end{array}$ \\
\hline $\begin{array}{c}\text { Moral Justifications } \\
\text { No Property }\end{array}$ & & & & \\
\hline $\begin{array}{r}p \\
\text { Cohen's } d \\
95 \% \mathrm{CI}\end{array}$ & --- & $\begin{array}{c}<.001^{*} \\
1.86 \\
{[0.56,0.73]}\end{array}$ & $\begin{array}{c}<.001^{*} \\
0.96 \\
{[0.30,0.57]}\end{array}$ & $\begin{array}{c}<.001^{*} \\
1.50 \\
{[0.47,0.69]}\end{array}$ \\
\hline $\begin{array}{r}\text { Intentions } \\
\text { Cohen’s } d \\
\mathrm{CI}\end{array}$ & --- & --- & $\begin{array}{c}<.001^{*} \\
-0.70 \\
{[-0.29,-0.13]}\end{array}$ & $\begin{array}{c}<.01^{*} \\
-0.31 \\
{[-0.12,-0.01]}\end{array}$ \\
\hline $\begin{array}{l}\text { Consent } \\
\qquad \begin{array}{r}p \\
\text { Cohen’s } d \\
\text { CI }\end{array}\end{array}$ & --- & --- & --- & $\begin{array}{c}<.001^{*} \\
0.42 \\
{[0.05,0.23]}\end{array}$ \\
\hline $\begin{array}{r}\text { Outcomes } \\
\text { Cohen's } d \\
\text { CI }\end{array}$ & --- & --- & --- & --- \\
\hline
\end{tabular}

Note. $*$ The mean difference is significant at the .01 level. 
In addition to the moral justification main effect, there was a significant interaction between condition and moral justification, Huynh-Feldt $F(7.73,440.60)=$ $5.55, \mathrm{p}<.001 ; \eta_{\mathrm{p}}^{2}=.09$. This interaction suggests that the type of condition had a bearing on the participants' moral justifications.

Abstract transgression condition. Pairwise comparisons (Bonferroni) revealed that the no properties justification appeared significantly more often than the outcomes $p<.001, d=6.20,95 \%$ CI $[0.83,1.08]$, intentions $p<.001, d=9.24$, CI [0.91, 1.04], and consent $p<.001, d=9.24$, CI [1.04] justifications. Thus, when participants responded to the abstract transgression condition, they most often relied on justifications that did not include moral properties language. Of the 41 no properties cases, authority and norms (34\%) and undefined (27\%) produced the greatest percentage of responses.

Intentions condition. When promoral intentions language was introduced to the scenario, the participants used the no property justification significantly more often than outcomes $p<.001, d=1.28$, CI $[0.24,0.78]$ and intentions $p<.001, d=1.41$, CI [0.30, 0.79]. The use of consent justifications was used significantly more often than intentions justifications $p=.01, d=0.73$, CI [0.03, 0.48] and approached statistical significance when compared to the outcomes justifications $p=.06, d=0.62$, CI [-0.01, 0.46]. Therefore, when the condition included a promoral intention for causing property destruction, the participants either did not use moral properties language or relied on consent as a justification. Of the 25 no properties cases, undefined justifications (28\%) received the greatest percentage of responses.

Consent condition. When promoral consent language was introduced in the scenario, the most frequently used justification was the no properties justification, which 
was used significantly more often than the outcomes $p<.001, d=1.53$, CI $[0.35,0.82]$ and intentions $p<.001, d=1.92, \mathrm{CI}[0.47,0.84]$ justifications. The consent justification was used significantly more often than the outcomes $p=.004, d=0.76$, CI $[0.07,0.51]$ and intentions $p<.001, d=1.06$, CI $[0.17,0.54]$. Thus, the participants' responses tended to include language centered on consent and explanations that did not include moral property language. Of the 30 no properties justifications, the authority and norms (53\%) and the undefined justifications (23\%) received the greatest percentage of responses.

Outcomes condition. When promoral outcomes language was introduced in the scenario, the use of the no properties justification was used more frequently than the outcomes $p=.001, d=1.02$, CI $[0.14,0.70]$ and intentions justifications $p<.001, d=$ 1.38, CI [0.29, 0.74]. In addition, consent justifications were used more frequently than the intentions justifications $p=.001, d=0.91$, CI $[0.10,0.52]$. Of the 24 no properties cases, the highest percentages of responses fell into the undefined (38\%), authority and norms (33\%), and principle (21\%) categories.

Contextualized prototype condition. In the contextualized prototype condition, in which the intentions, consent, and outcomes properties were explicitly directed towards immoral stances in the scenario, the no properties justification had the greatest proportional mean. It was used more often than the outcomes $p=.003, d=0.95$, CI [0.12, 0.72], consent $p=.001, d=0.99$, CI [0.13, 0.73], and intentions $p<.001, d=$ 1.41, CI[0.31, 0.77] justifications. Thus, most of the justifications did not include language centered on the moral properties. Of the 33 "no properties" cases, most of the responses fell into the authority and norms (33\%), principle (27\%), and undefined (21\%) categories. 
Overall, there was an interaction between type of condition and justification among the killing, hitting, and property destruction scenarios but not with the stealing scenario. The abstract transgression and the contextualized immoral prototype conditions produced similar qualitative justifications, which most often represented the no properties category. Specific language about the moral properties became more apparent in nonprototypical scenarios; however, there was no dominant property across all four scenarios.

\section{Discussion}

\section{Moral Judgment Ratings}

The statistically similar ratings between the abstract transgressions and the contextualized immoral prototypes suggest that the two conditions may share similar property structures. Thus, it is plausible that when moral transgressions are presented in the abstract, they activate default immoral prototypes that lead to inferences about bad intentions, a lack of consent, and negative consequences.

Although the abstract transgression and contextualized immoral prototype conditions resulted in similar judgment ratings when compared to each other, they did not produce the same consistent effects when compared to the three nonprototype conditions. The contextualized immoral prototypes were rated as more wrong than all of the nonprototypes. However, this was not true with the abstract transgressions. In the hitting and stealing scenarios, the abstract transgression baseline did not differ significantly from the promoral intentions condition. One potential reason for this discrepancy is that there is less ambiguity among the contextualized immoral prototypes, since the orientations of the properties were explicitly stated. Since the properties were not explicitly stated in the 
abstract cases, there may have been a few participants who interpreted the moral transgressions as subject to situational factors, such as "self-defense". Although, responses like "self-defense" or "it depends" were seldom used, this finding suggests that not all participants relied on default prototypes when presented with an abstract transgression.

The addition of promoral properties produced different moral judgment ratings, and when the promoral properties were added in a step-by-step process, the overall mean judgment shifted accordingly. In the killing and property destruction scenarios, there was a significant difference in judgment between the abstract transgression condition (Condition 1) and the intentions condition (Condition 2). For instance, in Condition 1, when participants were asked if it is wrong or all right to destroy property, the participants rated it as wrong. However, in Condition 2, when the actor destroys his neighbor's property because he is worried that it might hurt someone, the participants rated this condition as significantly less wrong than Condition 1. Additionally, there was a significant difference in judgment between the intentions condition (Condition 2) and the consent condition (Condition 3), which compared the promoral intentions with promoral intentions + consent. For example, in Condition 3 of the property destruction scenario, the actor not only wants to destroy the property to prevent it from hurting someone, but he also gets the consent of the owners. This condition was rated as significantly less wrong than Condition 2. Because there were two "jumps" in judgments as the ratings moved increasingly towards the "right" end of the continuum, these findings suggest that this combination of promoral intention and consent properties carried weight and produced additive effects. In the stealing and hitting scenarios, 
however, the significant shift did not happen until comparisons were made between Conditions 1 and 3. Thus, Condition 2 did not carry enough weight to shift judgments on its own in these two scenario sets. It may be that some immoral situations are more resistant to the effects of promoral properties. For example, in another study that included judgments about stealing, Posada and Wainryb (2008) interviewed Colombian children living in a violent, impoverished neighborhood. They found that the children expected people to steal to survive, but they also viewed the action as morally wrong; thus, even though the stealing was viewed as necessary, the children did not condone it. Some cases may require a significant accumulation of promoral weight before the judgments begin to shift.

Condition 3, which included both promoral intentions and consent, seemed to have the greatest impact on judgment when compared to both Condition 1 (the abstract transgression) or Condition 5 (the contextualized prototype conditions) (see Figure 4). 


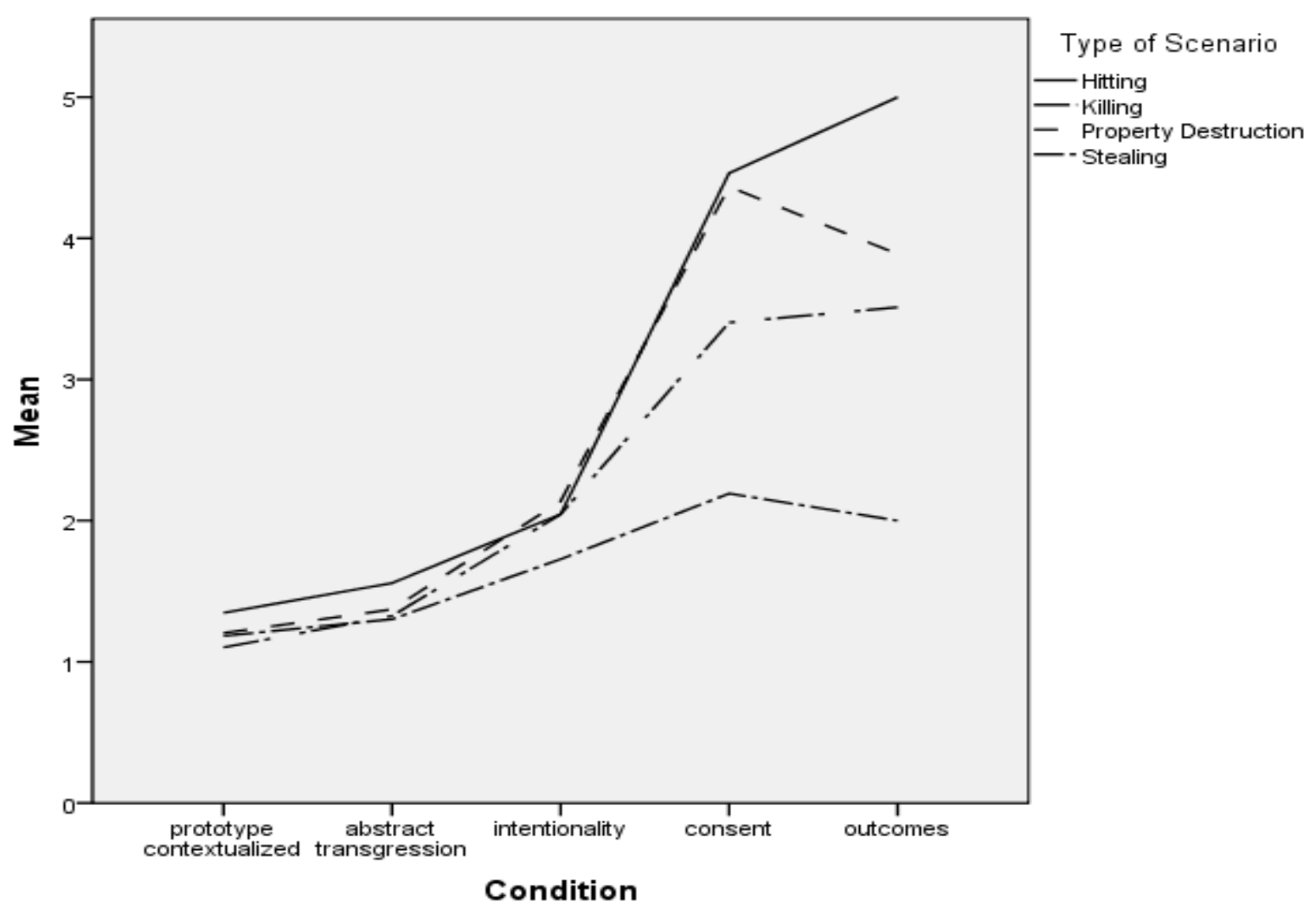

Figure 4. Line graph of each scenario’s moral judgment means by condition.

Across all four scenarios, the comparison between Conditions 1 and 3 resulted in a significant ratings shift. Cohen's $d$ effect sizes are considered to be large when they are greater than the absolute value of 0.80 and very large when they exceed the absolute value of 1.00. When the promoral intentions + consent condition was compared to the abstract transgression, that combination produced large effect sizes $d=0.80-2.38$ (in absolute values). Interestingly, adding a third promoral property to Condition 4 (i.e., explicit promoral intention + consent + outcomes) did not yield significant changes in judgments in any of the four scenarios when compared to Condition 3. Thus, there was a diminishing effect on judgment when the promoral outcome variable was added. One 
possible explanation for this result is that the participants may have anticipated or inferred what the outcomes would be, so the effects of the promoral outcomes were already integrated into their responses in Condition 3. For instance, in Condition 3 of the stealing scenario, a mother cannot afford to buy a winter coat for her daughter, so she takes one from the store. The shopkeeper knows the family's circumstances, sees the act, and permits it to happen. In Condition 4, the participant learns that the daughter stayed warm and healthy as a result of the coat. Because Condition 4 did not significantly differ from Condition 3, it is possible that the participants already inferred or anticipated that the coat would help the daughter in some way; thus, the weight of the outcomes property was integrated into the Condition 3 responses. An alternative interpretation could be that the added, explicitly promoral outcome balanced out the negative outcome associated with the moral transgression, resulting in less impact. For instance, in Condition 4 of the killing scenario, the doctor ends the patient's suffering, but the patient was also killed; thus, there are two outcomes in the end, one positive (end of suffering) and one negative (death of patient). If moral properties carry weight and judgments are affected by whether or not they are oriented towards a promoral or immoral stance, having two opposing outcomes might produce an averaging effect, which would result in the diminished salience of one or both outcomes. A third explanation may be that the effects of adding a combination of properties "tops off" at a certain point. In other words, there might be a maximum effect after a certain number or specific combination of properties is added. Finally, it is also plausible that some properties are viewed as more important than others. For instance, the intentions of the agent may have a stronger bearing on judgment than the outcomes in some cases. 
The shifting judgments in this study lend support to previous findings that show how situational factors, such as the intentions of the action, can lead to different moral judgments. For example, Turiel et al. (1991) found that when the reasons for an abortion varied, judgments also shifted. Additionally, when a father’s intentions for spanking a child changed from doing it for pleasure to helping the child, those judgments shifted accordingly as well (Wainryb, 1993a). These results suggest that people may judge an abstract moral transgression one way, but when a similar action is presented in context or when new information is added, the particulars of that situation can drive judgments into a different direction. The shifting judgments observed in these two previous studies and in this present research may be attributed to inferences people make about abstract moral situations. For example, if a person judges killing, as an abstract concept, to be wrong and then judges killing with good intentions as less wrong, then it can be deduced that the person may have inferred a different intention orientation in the abstract condition. Thus, the shifts in judgment that result from the moral property manipulations in this study support the hypothesis that people make inferences about moral properties when they are presented with abstract transgressions.

\section{Moral Judgment Frequencies and Percentages}

For the most part, the abstract transgression and contextualized prototype conditions resulted in perfect or near perfect agreement (98\% - 100\%) that the action was wrong. Among these two conditions, the hitting scenario's abstract transgression was the only one to elicit a "right" response from a participant. This participant justified the response by stating "self-defense”, which indicates that this person may have been thinking about the action under different circumstances while others seemed to perceive 
the hitting as an unprovoked act of aggression (e.g., "To inflict pain to an unsuspecting person is horrible"). These results confirm previous findings that show a high percentage of agreement in interpreting abstract hitting, stealing, property destruction, and killing as wrongful acts (Nucci, 1985; see Nucci, 2001 for discussion). In contrast, the nonprototype conditions appeared to produce greater divisions between judgments of right and wrong. For example, the good intentions condition across all four scenarios ranged from $84 \%$ - 96\% agreement that the situation was wrong. When promoral consent was added to promoral intentions, the responses ranged from $23 \%-79 \%$ agreement that the situation was wrong, and when promoral outcomes were added, the responses ranged from $13 \%$ - 91\% agreement that the situation was wrong. These

findings support the hypothesis that moral prototypes will generate moral agreement and nonprototypes can act as a source of disagreement.

\section{Moral Justifications}

The abstract transgression and the contextualized immoral prototype conditions produced similar qualitative justifications. Most of the responses fell into the no properties category, and the no property responses significantly outnumbered intentions, consent, and outcomes justifications. The only exception was in the contextualized immoral prototype of the hitting scenario in which there was no significant difference between the number of intentions and no properties justifications. Thus, in that condition, the participants provided intention and no properties justifications most often in their responses. The frequent occurrence of the no properties justification in these two conditions may be due to the lack of conflict between the properties. In our social context, the negative interpretations of hitting, property destruction, stealing, and killing 
may activate a default immoral prototype in which it is assumed or inferred that the act was performed with bad intentions, a lack of consent, and resulted in negative outcomes. When the properties of these actions are made explicitly promoral, such as stating that a doctor kills a patient because she wants to help the patient, a nonprototypical conflict is created. In the abstract and contextualized immoral conditions, promoral properties were not included; thus, in theory, there was no conflict. Without a conflict among the properties, there may have been less need to focus on them.

The no properties justifications in the abstract and contextualized immoral prototype conditions, when broken down into its subcategories, included a high proportion of undefined, principled, and authority/norms responses. For example, some of the participants stated, "Murder is illegal,” and “The bible says, 'No',” which briefly defer to legal and religious authority. The lack of conflict may have also led the participants to believe that the judgment did not require much elaboration. This would account for the large proportion of undefined, no properties responses (e.g., "Murder is bad,” and "Very unethical, immoral, and ugly.”).

Earlier, I proposed that the shift in judgment ratings might signify inferences being made about the moral properties when they are not explicitly stated. The qualitative findings also provide some evidence that support this hypothesis. For instance, in the intentions condition of the killing scenario, the doctor kills her patient, because she wants to end the patient's suffering. Some participants justified their reasons by stating that the patient did not consent to the killing, even though no explicit information about consent was presented in the scenario. Some example responses were: "Patient needs to be notified," “It was wrong, because Sarah didn’t ask for it," and "She 
did not have Sarah's consent to kill her.” In the consent condition of the hitting scenario, a woman is choking and gestures for her friend to hit her on the back. The friend, wanting to help the choking woman, forcefully punches her on the back in an attempt to dislodge the food. A few responses to this condition also suggest that some participants inferred the outcome, because the participants justified their judgments with an end result when no explicit language about the outcome was included in the scenario. One person said, “Tammi saved Karen’s life.” Another said, "She lived.” Since the majority of the responses did not include language that suggests that inferences were made, it is difficult to discern whether or not most people made inferences or if only a few did. Future studies should include interviews that ask participants specific questions about the moral properties in order to test whether or not most people do in fact make inferences about them when they are not explicitly stated.

The justification findings also show that when promoral language was added to the abstract transgression and a conflict was created (Conditions $2-4$ ), the participants started to include more specific language about the properties. Because there was no dominant property across all four scenarios, there is reason to believe that that the salience of a property can vary by scenario type. For example, consent was often used as a justification in the killing scenario and intentions and outcomes were often used in the hitting scenario. Interestingly, the moral property used in a given response did not appear to be dependent on the condition in some cases. For example, in the promoral intentions condition of the killing scenario, the doctor kills the patient because she cares about the patient and wants to help relieve her pain. Since the manipulation of that condition was the intentions of the doctor, I expected more people to include language about intentions 
in their responses. However, more participants expressed concerns about the lack of consent, even though no language about consent was explicitly stated in the scenario. This shows that the participant's justification was not simply the mirroring of the manipulated moral property. Instead, consent had a strong bearing on judgments in the killing nonprototypical conditions regardless of whether or not it was explicitly stated.

Although there was an interaction between condition type and justification among the killing, hitting, and property destruction scenarios, this was not the case with the stealing scenario. Instead, the no properties justification in the stealing scenario was used most frequently across all of the conditions. Because there was no interaction, no further post-hoc tests were performed on this scenario set. However, the frequency pattern of the no properties subcategories in the stealing scenario shows that there was a high proportion of undefined justifications across all five conditions. There was also a high proportion of principled and authority/norms justifications in the prototype conditions and a high proportion of counterfactual justifications among the nonprototype conditions. One reason for this high proportion of counterfactuals (>30\%) may be due to some participants' belief that the good intentions, consent, and/or outcomes were not enough to make the action acceptable, because the agent could have generated a more desirable, alternative solution. Thus, some participants felt that the woman should not have stolen the coat, because she could have acquired one through legitimate means. For example, one participant said, "Janine should have never stolen the jacket for her daughter. She could have asked for assistance." Another said, "Stealing is not the answer; there are agencies available that can help--a local church outreach program, the Division of Youth and Family Services, etc.” This finding suggests that there may be circumstances in 
which people also consider what options are available to the agent when making moral judgments.

\section{Limitations and Suggestions for Future Research}

The primary goal of Study 1's exploratory research was to compare abstract transgressions and contextualized immoral prototypes to see if they act similarly and to observe whether or not the addition of promoral properties would carry enough weight to shift judgments from "wrong” to "right". As a result, this study began with a baseline stimulus that reflected an immoral orientation and then attempted to manipulate the baseline by adding promoral properties. One limitation is that the properties were not counterbalanced. The reason for this was due to budgetary constraints.

Counterbalancing the properties would have required more questions and higher costs, since subject pool services typically base their fees on the number of questions and participants in the study. In order to make the study affordable, I made the decision not to counterbalance the properties and reduce the number of test questions. By testing multiple scenarios, I was given an opportunity to look for patterns across different situations. Although there was no theoretical support for the order in which I added the properties, a consistent order was maintained for the purpose of creating a control for the experiment. As a result of this limitation, future studies should counterbalance the explicitly added properties to test which single properties and combination sets produce the greatest effects. In this study, adding promoral outcomes in Condition 4 did not produce any significant effects. Was this simply due to the order that it was added? Or was it because promoral outcomes don't carry as much weight as promoral consent and intentions? Counterbalancing will help to answer these questions. It will also provide 
more information about dominant properties. For example, consent was the most salient property used in justifications of the killing scenario. By counterbalancing the properties and beginning with consent, would consent produce the greatest effect in the killing scenario if it is added first, and would there be diminishing effects after the other properties are added?

In addition, a fuller understanding of the interplay between promoral and immoral properties could be achieved by reversing the pattern (i.e., creating a promorally oriented baseline and observing the effects of adding immoral properties in the nonprototype conditions). Because there is a documented moral judgment asymmetry between promoral and immoral situations (Ohtsubo, 2007; Pizarro, Uhlmann, and Salovey, 2003), it seems reasonable to predict that the effects would differ. The results of Study 2 suggest that people are more sensitive to immoral properties than to promoral properties; thus, there might be a strong shift in judgment with the addition of the first immoral property to the promoral baseline.

Finally, future studies should include alternative research methods and designs. The consecutive shifts in the judgments of the killing and property destruction scenarios suggest that there may be a linear relationship between the weight of a moral property and the moral judgment rating in some situations. Using methods, such as trend analyses, that look at the functional relationship between the variables could provide a richer understanding of how the orientation and accumulated weight of moral properties affect moral judgments. To supplement the quantitative data, including interviews will provide a better means for studying whether or not inferences about the orientations of the 
properties inform judgment, since there was no opportunity to ask follow up questions to the Internet responses.

\section{Conclusion for Study 1}

In sum, the findings in this study suggest that the abstract transgressions and contextualized prototypes generate similar judgments and result in perfect or near perfect agreement that the situation is wrong. Greater disagreement appears to become manifest when people judge nonprototypes. In general, the abstract transgressions and contextualized prototypes were often judged to be more wrong than the nonprototypes but never judged to be more right. Additionally, this study found that the promoral intentions and consent properties appear to carry weight and may produce additive affects when they are combined. The shifting judgments in this study support the hypothesis that inferences are made when the properties are not made explicit. Although the justifications provided additional evidence that the participants inferred the orientation of an inexplicit property or relied on a default immoral prototype when presented with an abstract transgression, more interview-centered research is needed to further test this hypothesis. Finally, the findings in this study suggest that justification language will vary according to how a moral situation is presented. 


\section{CHAPTER IV: STUDY 2}

\section{Method}

\section{Purpose}

The concept of moral prototypes is based on the premise that categories of what is morally wrong or right consist of the following properties: social context, intentions, consent, and outcomes. When the properties are uniformly directed towards immoral stances (e.g., bad social context, bad intention, no consent, and bad outcomes), an immoral prototype will result. When they are uniformly directed towards promoral stances (e.g., good social context, good intentions, full consent, and good outcomes), a promoral prototype will result. Nonprototypes deviate from the prototype and may not fit neatly into a particular category.

The purpose of this study was to create nonprototypes by manipulating the orientations of the four properties and observing whether or not moral judgement would differ between prototypes and nonprototypes. It was predicted that the following results would become evident:

1. Prototypes will differ from nonprototypes according to levels of right/wrong, praise/blame, and difficulty.

2. Prototypes will result in a perfect or near perfect percentage of moral agreement.

3. Moral property justification language will vary according to the orientations of the moral properties.

\section{Pilot Study}

Prior to collecting the final data for this study, a pilot study was conducted to assess the following: (1) whether or not the social context variables would be interpreted 
as having a general immoral or promoral stance, and (2) if the participants could identify the manipulated variable via a manipulation check.

Sixty-eight students taking educational psychology courses at the University of Illinois at Chicago volunteered to participate in this pilot study. Of the 68 students (Mage $=23, M d n=22$, range $=19-39, S D=4.06)$ who provided this information, $48(71 \%)$ were women and 20 (29\%) were men. Two (3\%) of the participants identified themselves as African-American, 10 (15\%), Asian, 17 (25\%), Latino, and 38 (56\%) as Caucasian. Fifty-three (78\%) participants were born in the U.S. while 14 (21\%) were born in a different country. One participant did not provide information about race or birth country.

In Part I of this pilot study, the goal was to determine if the social context variables would result in general agreement. The participants were asked to make a judgment of right or wrong concerning the following actions: marrying someone you love, marrying someone you find disgusting; raising money for charity by collecting volunteer donations, raising money for charity by holding a baby auction; hiring an adult to work in a factory, hiring a 5-year-old boy to work in a factory; and creating an affordable drug for profit, selling human body parts for profit. The responses to these items ranged from 90 percent - 100 percent agreement that the action was either right or wrong (see Table XI). This high level of agreement suggests that the social context variables are likely to produce the intended effect of carrying either a general promoral or immoral stance within each scenario. 
TABLE XI

RESULTS OF SOCIAL CONTEXT VARIABLE AGREEMENT

\begin{tabular}{|c|c|c|c|}
\hline Social Context Variables & $n$ & $\begin{array}{c}\text { Frequency and } \\
\text { (Percentage) of } \\
\text { those who reported } \\
\text { that the action is } \\
\text { "wrong" }\end{array}$ & $\begin{array}{c}\text { Frequency and } \\
\text { (Percentage) of those } \\
\text { who reported that the } \\
\text { action is "right" }\end{array}$ \\
\hline marrying someone you love & 68 & 0 & $68(100)$ \\
\hline $\begin{array}{l}\text { marrying someone you find } \\
\text { disgusting }\end{array}$ & 68 & $61(90)$ & $7(10)$ \\
\hline $\begin{array}{l}\text { raising money by collecting } \\
\text { volunteer donations }\end{array}$ & 67 & $2(3)$ & 65 ( 97) \\
\hline $\begin{array}{l}\text { raising money by holding a baby } \\
\text { auction }\end{array}$ & 68 & $68(100)$ & 0 \\
\hline $\begin{array}{l}\text { hiring an adult to work full-time } \\
\text { in a factory }\end{array}$ & 68 & $1(2)$ & 67 ( 99) \\
\hline $\begin{array}{l}\text { hiring a 5-year-old to work full- } \\
\text { time in a factory }\end{array}$ & 68 & $68(100)$ & 0 \\
\hline creating an affordable drug & 68 & $1(2)$ & 67 ( 99) \\
\hline selling human body parts & 67 & $60(90)$ & $7(10)$ \\
\hline
\end{tabular}

Note: Due to rounding, some percentages may not add to 100 . Unequal $n$ is due to missing data.

The goal of the second assessment was to determine whether or not the participants would be able to identify the manipulated variable in each scenario. Due to the length of the pilot study and classroom time constraints, two separate forms of the manipulation check were administered in order to provide time for the participants to respond to each question. Thirty of the 68 participants completed Form A, which included one promoral scenario involving a man who helps a homeless person obtain factory work and one immoral scenario about a group of scientists who sold cloned human organs for profit. Thirty-five participants completed Form B, which included a promoral scenario about a woman who helps orphaned children and an immoral scenario 
about a man and his desire to marry a person he does not love. (Three participants were dropped for not completing the manipulation check on either form.)

In this section, the participants first read a scenario that represented either a promoral or immoral prototype in which there was no variable manipulation. The participants were then asked to compare the prototype scenario to four nonprototype scenarios, in which a variable (i.e., social context, intentions, consent, or outcomes) was manipulated and directed towards an opposing stance. In other words, the conflict-free prototype, in which all the variables share the same orientations, was compared to a series of nearly identical nonprototypical scenarios. In each, one of the variables was redirected towards the opposing stance. The participants were then asked to identify how the nonprototypical scenario differed from the prototype. Since it was anticipated that some of the participants might not be familiar with the terminology specific to the study, multiple-choice responses were presented in laymen's terms in order to improve the participant's comprehension. A sample question is as follows:

Compare Scenario 1 with Scenario 2. The two scenarios are different because: whether or not Fred loves Anne is not the same. Fred's intentions for marrying Anne are not the same. whether or not Anne agrees to marry Fred is not the same. how Anne feels after the marriage is not the same.

The responses to the scenarios in both Form A and Form B ranged from 89 - 100 percent accuracy in the participants' ability to detect the manipulated variables (see Table XII). Thus, the variables were correctly identified by at least 89 percent of the participants, and each of the piloted scenarios were used for the final data collection. 
TABLE XII

RESULTS OF VARIABLE MANIPULATION CHECK

\begin{tabular}{|c|c|c|c|}
\hline Scenario & $n$ & $\begin{array}{l}\text { Frequency and } \\
\text { (Percentage) of those } \\
\text { who correctly } \\
\text { identified the } \\
\text { manipulated variable } \\
\end{array}$ & $\begin{array}{c}\text { Frequency and } \\
\text { (Percentage) of those } \\
\text { who did not correctly } \\
\text { identify the } \\
\text { manipulated variable }\end{array}$ \\
\hline \multicolumn{4}{|l|}{ Form A } \\
\hline \multicolumn{4}{|l|}{ Cloning } \\
\hline Social Context & 30 & 28 ( 93) & $2(7)$ \\
\hline Intentions & 30 & $30(100)$ & 0 \\
\hline Consent & 30 & 29 ( 97) & $1(3)$ \\
\hline Outcomes & 30 & 29 ( 97) & $1(3)$ \\
\hline \multicolumn{4}{|l|}{ Factory Work } \\
\hline Social Context & 30 & 28 ( 93) & $2(7)$ \\
\hline Intentions & 30 & 29 ( 97) & $1(3)$ \\
\hline Consent & 30 & 29 ( 97) & $1(3)$ \\
\hline Outcomes & 30 & 29 ( 97) & $1(3)$ \\
\hline \multicolumn{4}{|l|}{ Form B } \\
\hline \multicolumn{4}{|l|}{ Marriage } \\
\hline Social Context & 35 & 31 ( 89) & $4(11)$ \\
\hline Intentions & 35 & 33 ( 94) & $2(6)$ \\
\hline Consent & 35 & 33 ( 94) & $2(6)$ \\
\hline Outcomes & 35 & 33 ( 94) & $2(6)$ \\
\hline \multicolumn{4}{|l|}{ Orphanage } \\
\hline Social Context & 35 & 34 ( 97) & $1(3)$ \\
\hline Intentions & 35 & $35(100)$ & 0 \\
\hline Consent & 35 & 35 (100) & 0 \\
\hline Outcomes & 35 & $35(100)$ & 0 \\
\hline
\end{tabular}

Note: Due to rounding, some percentages may not add to 100.

\section{Participants}

The participants were recruited by a service that provides researchers and marketing specialists with subject pools consisting of adults (18 years and older) who have enrolled in a rewards program. Points are earned from responding to questionnaires and later redeemed for premiums (e.g., coupons or gift certificates). The participants are directed to the service via Internet ads and, once registered, receive descriptions and links 
to a number of studies. They choose their questionnaires and are given the opportunity to unsubscribe to the program or opt-out from any study at any time without consequences.

In this research study, the recruiting service provided potential participants with a link to my questionnaire. Before each person was allowed to view and respond to it, the person was asked to read and agree to an online consent form. If a person chose not to consent to the research, the web site redirected the visitor to an exit page.

In order to achieve a minimum power of .80 and to detect a .25 effect size (in standard deviation units) with an alpha of .05, I estimated that approximately 400 participants would be needed for this MANOVA study. In total, 489 people responded to the questionnaire. Seven participants were dropped for providing “1's” across all quantitative responses and for providing unusable responses for each qualitative section, such as "bbbb" or "aarenc". In order to control for cultural and developmental variables, the participants were limited to adults (18 years or older), who were born and are currently living in the United States. Of the 481 remaining participants (Mage $=44, M d n$ $=44$, mode $=54$, range $=18-80, S D=14.26 ; M$ income $=\$ 52,278, M d n=46,000$, mode $=$ 50,000, range $=0-120,000, S D=36,319.04), 215(45 \%)$ are male and $266(55 \%)$ are female. Self-reported ethnic/racial identities included the following: 61 (13\%) Asian, 89 (19\%) Black or African American, 77 (16\%) Hispanic or Latino, 202 (42\%) are White or Caucasian, 43 (9\%) Native American, and 9 (2\%) Other. The highest level of education included 156 (32\%) participants who completed high school, 142 (30\%) who completed an associates or vocational program, 129 (27\%) who completed a bachelors program, and 54 (11\%) who completed a graduate degree. 


\section{Measures and Procedures}

In this study, I asked participants to read scenarios that represented moral

prototypes or nonprototypes (see Appendix for full question set). An example of a situation that represents a moral prototype is the following:

Beth is a devoted advocate for orphaned children. Recently, she created a foundation that helps them find good homes. Voluntary donations are used to take care of the orphans while they are waiting to be adopted. The children and the community value her contribution and are grateful for the assistance it provides. Because of Beth's charity work, the orphans and their new families are living happier, healthier lives.

In this example, the properties of the prototype are coherent and represent a promoral prototype in which each property is oriented towards a promoral stance. The social context condones charity work, the intention is benevolent, there is consent, and the intentional action results in positive outcomes for children.

To create scenarios that represent instances that deviate from the prototype (i.e., nonprototypes), one property was systematically manipulated and directed toward the opposing orientation while the other features were controlled to remain constant. For instance, beginning with a promoral prototype in which there is coherence among the properties, the intentions variable was redirected towards an immoral stance for the purpose of producing a "bad" intentions conflict, as in the following example:

Beth recently created a foundation that helps orphaned children find good homes. Voluntary donations are used to take care of the children while they are waiting to be adopted. Beth hates orphaned children and finds them revolting. 
However, she hopes to run for public office and plans to use her charity work to convince more people to vote for her. The children and the community value her contribution and are grateful for the assistance it provides. Because of Beth's charity work, the orphans and their new families are living happier, healthier lives.

In this example, the social context, consent, and outcomes properties were each directed towards promoral stances. However, the intentions property (i.e., Beth using the charity work for her own personal gains) was directed towards an immoral stance; thus, intentions was in conflict with the other properties.

In a second example, the consent property was oriented towards an immoral stance to produce a "bad" consent conflict as depicted in the following scenario:

Beth is a devoted advocate for orphaned children. Recently, she created a foundation that helps them find good homes. Voluntary donations are used to take care of the children while they are waiting to be adopted. Although Beth wants to help the orphans, the children and the community do not want her services. They want to develop their own solutions to the orphan problem. Beth believes that her services are necessary, so she finds a way to force the children and the community to accept her services against their will. Because of Beth’s charity work, the orphans and their new families are living happier, healthier lives.

Right/Wrong. In order to test for differences in judgments of right or wrong, the participants were asked to make a judgment by rating the level of right or wrong according to a 6 -point, bipolar scale ( $1=$ very wrong $-6=$ very right). The neutral 
category (i.e., "neither agree nor disagree”) was omitted in order to require participants to make a judgment and to reduce the effects of over-relying on the neutral category (Linacre, 2004; Stone, 2004). An open-ended question that asked the participants to explain their answers was used to collect qualitative responses. It was predicted that promoral prototypes would produce a high degree of "right” judgements and immoral prototypes would produce a high degree of "wrong” judgements. It was also predicted that nonprototypical items would produce a greater range of responses (i.e., an increased likelihood for moral disagreement).

A five-category coding scheme was used to analyze the open-ended justification responses. The categories included the four proposed moral properties (i.e., social context, intentions, consent, and outcomes) and a "no property" category, which was used for responses that did not contain any moral property language (see Table XIII for category descriptions and sample responses). A graduate student and I coded $20 \%$ of the responses using this scheme and obtained an interrater agreement level of .90 (Cohen’s Kappa). When there was disagreement, we talked about the response until we reached an agreement about how to categorize it. 


\section{TABLE XIII \\ DEFINITIONS AND SAMPLES OF JUSTIFICATION CATEGORIES}

\begin{tabular}{|c|c|c|}
\hline Category & Definition & Sample Responses \\
\hline Social Context & $\begin{array}{l}\text { Judgements that include language about the } \\
\text { following social context variables: marrying for } \\
\text { love/not marrying for love; creating medical drugs } \\
\text { for profit/harvesting cloned body parts for profit; } \\
\text { hiring an adult for factory work/hiring a 5-year- } \\
\text { old for factory work; and collecting volunteer } \\
\text { donations to raise money for a charity that helps } \\
\text { orphans/auctioning orphans to raise money for a } \\
\text { charity that helps orphans. }\end{array}$ & $\begin{array}{l}\text { "I do not think that his actions were } \\
\text { correct because of the age of the boy." } \\
\text { "...cloning, even of your own body } \\
\text { parts, is just morally reprehensible to } \\
\text { me." } \\
\text { "I think that you should be totally in } \\
\text { love when you marry..." }\end{array}$ \\
\hline Intentions & $\begin{array}{l}\text { Judgements based on the perceived intentions of } \\
\text { the actor. }\end{array}$ & $\begin{array}{l}\text { "Their intentions are good." } \\
\text { "His actions and reasons were very } \\
\text { wrong and selfish on his behalf." } \\
\text { "Beth's intentions to use the charity to } \\
\text { her benefit is wrong." }\end{array}$ \\
\hline Consent & $\begin{array}{l}\text { Judgments influenced by whether or not the act } \\
\text { was seen as a matter of personal choice and/or } \\
\text { whether or not the target of the action was a } \\
\text { willing participant. }\end{array}$ & $\begin{array}{l}\text { "It is not okay to experiment on } \\
\text { people without their knowledge nor } \\
\text { on minors without parental consent." } \\
\text { "No one should have anything forced } \\
\text { on them, period!" } \\
\text { "The coercion of the man was the } \\
\text { wrong tact." }\end{array}$ \\
\hline Outcomes & $\begin{array}{l}\text { Judgments that focus on the actual, inferred, or } \\
\text { predicted outcomes of the action. }\end{array}$ & $\begin{array}{l}\text { "Wrong! They caused the deaths.” } \\
\text { “The outcome worked for both.” } \\
\text { “The end result justifies Beth's } \\
\text { actions.” }\end{array}$ \\
\hline No Property & $\begin{array}{l}\text { Judgements that do not contain any language } \\
\text { about moral properties. }\end{array}$ & $\begin{array}{l}\text { "Ann was stupid." } \\
\text { "This is what the human race is } \\
\text { about." } \\
\text { "It is hard to say, maybe family } \\
\text { interviewing should be more stricter." } \\
\text { "They were wrong." }\end{array}$ \\
\hline
\end{tabular}


Due to the large number of "no property" responses, a second coding scheme was developed by using categories from previous research on moral judgment (Brehl, 2008; Nucci \& Turiel, 1993). The aim of this coding scheme was to find out how participants justified their moral judgments when they did not use language about social context, intentions, consent, or outcomes. After running the main coding scheme across all responses, the no property cases were then selected and subject to the second coding scheme, which included the following categories: authority and norms, principled justifications, contingent justifications, counterfactuals, undefined responses, and uncodable responses (refer back to Table II for descriptions and sample responses). A graduate student and I coded 20 percent of the no property responses for reliability and obtained an interrater agreement level of .94 (Cohen’s Kappa).

Per participant, each written justification was coded as a single response. If more than one category was present in a response, it was partitioned into proportional values. For example, if a response contained language relevant to consent, outcomes, and intentions, each of those categories received a value of .33.

Difficulty. In addition, the participants were asked to rate the difficulty level of each scenario by responding to a 5 -point scale that ranged from $1=$ not difficult $-5=$ extremely difficult. It was predicted that the prototypes would be easier to judge and the nonprototypes would be more difficult to judge.

Praise/Blame. Finally, the participants were asked to make a judgement by rating the level of praise or blame according to a 5-point, bipolar scale ( 1 = full blame, 3 $=$ no praise or blame, $5=$ full praise). It was predicted that the promoral prototypes would receive a greater degree of praise and the immoral prototypes would receive a 
greater degree of blame. It was also expected that the nonprototypes would produce a greater range of responses.

\section{Results}

\section{Data Analysis Plan and Data Reduction}

Moral Judgment Ratings. Because exploratory data analyses showed that the three dependent variables were correlated (see Table XIV), the analysis was conducted by comparing type of condition (i.e., social context, intentions, consent, outcomes, and prototype) in a between groups, one-way MANOVA design. MANOVA, as opposed to performing a separate ANOVA on each dependent variable, offers protection against an inflated Type I error rate. 
TABLE XIV

PEARSON CORRELATIONS OF DEPENDENT VARIABLES

\begin{tabular}{|c|c|c|c|}
\hline \multirow[b]{2}{*}{$\begin{array}{l}\text { Scenario } \\
\text { Dependent Variable }\end{array}$} & \multicolumn{3}{|c|}{ Dependent Variable } \\
\hline & Wrong or Right & Praise or Blame & Difficulty Level \\
\hline \multicolumn{4}{|l|}{ Cloning } \\
\hline Wrong or Right & 1 & $.71^{* * *}$ & $.36 * * *$ \\
\hline Praise or Blame & - & 1 & $.44 * * *$ \\
\hline Difficulty Level & -- & -- & 1 \\
\hline \multicolumn{4}{|l|}{ Marriage } \\
\hline Wrong or Right & 1 & $.68^{* * *}$ & $.33 * * *$ \\
\hline Praise or Blame & -- & 1 & $.22 * * *$ \\
\hline Difficulty Level & -- & -- & 1 \\
\hline \multicolumn{4}{|l|}{ Factory Work } \\
\hline Wrong or Right & 1 & $.86 * * *$ & $-.28 * * *$ \\
\hline Praise or Blame & -- & 1 & $-.24 * * *$ \\
\hline Difficulty Level & -- & -- & 1 \\
\hline \multicolumn{4}{|l|}{ Orphanage } \\
\hline Wrong or Right & 1 & $.85^{* * *}$ & $-.11 *$ \\
\hline Praise or Blame & - & 1 & $-.10 *$ \\
\hline Difficulty Level & -- & -- & 1 \\
\hline
\end{tabular}

** Correlation is significant at the $<.001$ level (two-tailed).

*Correlation is significant at the .05 level (two-tailed). 
Previous research has found that there is an asymmetry between positive and negative actions (Malle and Knobe, 1997; Ohtsubo, 2007; Pizarro, Uhlmann, and Salovey, 2003). Additionally, the exploratory data analyses revealed that the scenarios used in this study are separate constructs (see Table XV).

TABLE XV

PEARSON CORRELATIONS OF SCENARIOS' LEVELS OF RIGHT/WRONG WITHIN EACH TREATMENT CONDITION

\begin{tabular}{|c|c|c|c|c|}
\hline \multirow[b]{2}{*}{$\begin{array}{l}\text { Treatment Condition } \\
\text { Scenario }\end{array}$} & \multicolumn{3}{|c|}{ Scenario } & \multirow[b]{2}{*}{ Orphans } \\
\hline & Cloning & Marriage & Factory Work & \\
\hline \multicolumn{5}{|l|}{ Social Context } \\
\hline Cloning & 1 & -.08 & .31 & .12 \\
\hline Marriage & -- & 1 & .04 & -.15 \\
\hline Factory Work & -- & - & 1 & $.45^{*}$ \\
\hline Orphans & -- & -- & -- & 1 \\
\hline \multicolumn{5}{|l|}{ Intentionality } \\
\hline Cloning & 1 & $.37 *$ & -.15 & -.10 \\
\hline Marriage & -- & 1 & $-.34 *$ & -.08 \\
\hline Factory Work & -- & -- & 1 & .31 \\
\hline Orphans & -- & -- & -- & 1 \\
\hline \multicolumn{5}{|l|}{ Consent } \\
\hline Cloning & 1 & .21 & .06 & .07 \\
\hline Marriage & -- & 1 & .05 & .08 \\
\hline Factory Work & -- & - & 1 & $.37 *$ \\
\hline Orphans & -- & -- & -- & 1 \\
\hline \multicolumn{5}{|l|}{ Outcomes } \\
\hline Cloning & 1 & .16 & .01 & .17 \\
\hline Marriage & -- & 1 & .02 & .08 \\
\hline Factory Work & -- & -- & 1 & $.38 *$ \\
\hline Orphans & -- & -- & -- & 1 \\
\hline \multicolumn{5}{|l|}{ Prototype } \\
\hline Cloning & 1 & .13 & .07 & -.15 \\
\hline Marriage & - & 1 & -.14 & -.15 \\
\hline Factory Work & -- & -- & 1 & -.07 \\
\hline Orphans & -- & -- & -- & 1 \\
\hline
\end{tabular}

*Correlation is significant at the Bonferroni adjusted 0.002 level (two-tailed). 
Thus, none of the scenarios were omitted or combined. Because it was not the aim of this research to determine if cloning is more wrong than marrying without love or if offering factory work is more right than running a charity for orphans, the four items were not tested and analyzed independently in four separate one-way MANOVA's. The core design is depicted by Figure 5, with the bolded property indicating the manipulated property in conflict.

\begin{tabular}{|l|l|l|l|l|}
\hline $\begin{array}{c}\text { Group 1: } \\
\text { Social Context } \\
\text { Conflict }\end{array}$ & \multicolumn{1}{|c|}{$\begin{array}{c}\text { Group 2: } \\
\text { Intentions } \\
\text { Conflict }\end{array}$} & $\begin{array}{c}\text { Group 3: } \\
\text { Consent } \\
\text { Conflict }\end{array}$ & $\begin{array}{c}\text { Group 4: } \\
\text { Outcomes } \\
\text { Conflict }\end{array}$ & $\begin{array}{c}\text { Group 5: } \\
\text { Prototype } \\
\text { (No Conflict) }\end{array}$ \\
\hline Social Context & Social Context & Social Context & Social Context & Social Context \\
Intention & Intention & Intention & Intention & Intention \\
Consent & Consent & Consent & Consent & Consent \\
Outcomes & Outcomes & Outcomes & Outcomes & Outcomes \\
\hline
\end{tabular}

Figure 5. Structure of the MANOVA research design by groups.

Applying transformation algorithms and the removal of outliers did not improve the skewed distributions nor yield different conclusions; therefore, the outliers were maintained to increase power, and the untransformed data were analyzed for the benefit of improving interpretation. In order to control for Type 1 error across the multiple tests, a Bonferroni adjustment was made and the alpha was set at .01 (two-tailed). Preliminary MANCOVA analyses were performed on each scenario set to test whether or not there were significant demographic related interactions or main effects on the right/wrong, praise/blame, and difficulty level dependent variables. The following independent 
variables were tested: type of condition, race, sex, and education with age and income acting as the covariates. Using Pillai's Trace, main effects and interactions of the demographic variables were not significant, $F=.230-2.707, p \geq .05, n s$. In addition, the covariates were not significant, $F=.394-3.555, p \geq .02$, ns. As a result, the demographic variables were dropped from subsequent analyses. In order to control for learning effects and testing fatigue, the participants were randomly assigned into the following 5 groups: Group 1 - social context conflict items; Group 2 - intentions conflict items; Group 3 - consent conflict items; Group 4 - outcomes conflict items; Group 5 prototype items.

Moral Judgment Frequencies and Percentages. In addition to the four, one way MANOVA’s, the frequencies and percentages of right and wrong responses were noted to discern the level of moral agreement in each condition. If a participant rated the scenario as "very wrong”, "wrong”, or "somewhat more wrong than right”, the judgment was coded as 1 = "wrong”. If the participant rated the scenario as "very right”, "right”, or “somewhat more right than wrong”, then the judgment was coded as 2 = "right". Then, the total number and percentages of "wrong” or "right” responses were computed. A high percentage of one category indicates agreement (e.g., 100\% of the responses were coded as “wrong”), while more proportionally divided responses indicate disagreement (e.g., 30\% responded “right” and 70\% as “wrong”).

Moral Justifcations. As a result of the between-groups design, proportional data, and cells with values of 0 , a decision was made to run an ANOVA. In order to analyze the data, a 5 condition (social context, intentions, consent, outcomes, prototype) x 5 moral justification (no property, social context, intentions, consent, and outcomes) 
mixed model, repeated measures ANOVA was run separately on each of the four scenarios (i.e., cloning, marriage, factory work, and orphans), with the justification codes serving as the repeated measures.

Since the framework of this proposed model of moral judgment compares moral prototypes with nonprototypes, hypothesis testing was not conducted on the data generated from the no property responses. Instead, I consider this data to be supplemental information for the reader. Thus, only the frequencies and percentages will be reported for the subcategories of the no property responses (see results in Table XVI). The subcategories that result in 20 percent of the responses or greater will be noted along with the ANOVA results. 
TABLE XVI

SUBCATEGORIES OF JUSTIFCATIONS CODED AS "NO PROPERTY” ACTUAL NUMBER (\% OF TREATMENT GROUP) [\% OF TOTAL] PER SCENARIO

\begin{tabular}{|c|c|c|c|c|c|c|}
\hline \multirow[b]{2}{*}{$\begin{array}{c}\text { Moral } \\
\text { Justifications }\end{array}$} & \multicolumn{6}{|c|}{ Condition } \\
\hline & $\begin{array}{l}\text { Social } \\
\text { Context } \\
\end{array}$ & Intentions & Consent & Outcomes & Prototype & Total \\
\hline \multicolumn{7}{|l|}{ Cloning } \\
\hline $\begin{array}{l}\text { Authority and } \\
\text { Norms }\end{array}$ & 7 (25) [ 6] & 2 (14) [ 2] & 0 & 2 (11) [ 2] & 3 (11) [ 3] & 14 [13] \\
\hline Principle & 3 (11) [ 3] & 0 & 0 & $2(11)[2]$ & $2(7)[2]$ & $7 \quad[6]$ \\
\hline Counterfactual & 1 ( 4) [ 1] & $1(7)[1]$ & $3(13)$ [ 3] & $1(6)[1]$ & $2(7)[2]$ & 8 [ 7] \\
\hline Contingency & 0 & 0 & $1(4)[1]$ & 2 (11) [ 2] & 0 & 3 [ 3$]$ \\
\hline Undefined & $16(57)[14]$ & $10(71)[9]$ & 17 (73) [15] & $11(61)[10]$ & $21(75)[18]$ & 75 [68] \\
\hline Uncodable & $1(4)[1]$ & $1(7)[1]$ & 2 ( 9) [ 2] & 0 & 0 & $4 \quad[4]$ \\
\hline Total & $28 \quad[25]$ & [13] & [21] & {$[16]$} & [25] & $\mathrm{N}=111$ \\
\hline \multicolumn{7}{|l|}{ Marriage } \\
\hline $\begin{array}{l}\text { Authority and } \\
\text { Norms }\end{array}$ & 1 (4) [ 1] & 0 & 0 & 0 & 0 & 1 [ 1] \\
\hline Principle & $1(4)[1]$ & $4(17)[4]$ & $1(7)[1]$ & 0 & $1(4)[1]$ & $7 \quad[6]$ \\
\hline Counterfactual & 5 (20) [ 5] & $5(22)[5]$ & $1(7)[1]$ & 3 (13) [ 3] & 1 ( 4) [ 1] & 15 [14] \\
\hline Contingency & 0 & $1(5)[1]$ & 0 & 3 (13) [ 3] & 0 & 4 [ 4$]$ \\
\hline Undefined & $15 \quad(60)[14]$ & $10(44)$ [ 9] & $9(60)[8]$ & 14 (58) [13] & 20 (87) [18] & 68 [62] \\
\hline Uncodable & 3 (12) [ 3] & 3 (13) [ 3] & $4(27)[4]$ & 4 (17) [ 4] & $1(4)[1]$ & 15 \\
\hline Total & 25 & [21] & [14] & {$[22]$} & {$[21]$} & $\mathrm{N}=110$ \\
\hline \multicolumn{7}{|c|}{ Factory Work } \\
\hline $\begin{array}{l}\text { Authority and } \\
\text { Norms }\end{array}$ & $4(13)\left[\begin{array}{l}2\end{array}\right]$ & 0 & $1(3)[1]$ & 0 & $1(2)[1]$ & 6 [ 4$]$ \\
\hline Principle & $5(16)\left[\begin{array}{l}3\end{array}\right]$ & 3 (21) [ 2] & $9(31)$ [ 5] & $9(19)$ [ 5] & 13 (27) [ 8] & 39 [23] \\
\hline Counterfactual & $3(10)$ [ 2$]$ & 0 & 0 & 0 & 0 & 3 [ 2] \\
\hline Contingency & 0 & 0 & 0 & $1(2)[1]$ & 0 & $1[1]$ \\
\hline Undefined & $18(58)[11]$ & 7 (50) [ 4] & $15(52)$ [ 9] & 35 (75) [21] & 33 (67) [19] & 108 [64] \\
\hline Uncodable & $1(3)[1]$ & $\left.\begin{array}{ll}4 & (29)\end{array}\right]$ & $2(4)[1]$ & $2(4)[1]$ & $2(4)[1]$ & 13 [ 8] \\
\hline Total & $31 \quad[18]$ & 14 [9] & [28] & [28] & [29] & $\mathrm{N}=170$ \\
\hline \multicolumn{7}{|l|}{ Orphanage } \\
\hline $\begin{array}{l}\text { Authority and } \\
\text { Norms }\end{array}$ & 0 & 0 & $1(4)[1]$ & 0 & $1(3)[1]$ & 2 [ 1] \\
\hline Principle & 0 & 0 & $3(13)[$ [ 2] & $4(8)[3]$ & $1(3)[1]$ & $8[6]$ \\
\hline Counterfactual & $1(5)[1]$ & 0 & $1(4)[1]$ & $13(26)[9]$ & 0 & $15[10]$ \\
\hline Contingency & $3(14)[2]$ & 0 & $1(4)[1]$ & $8(16)$ [ 6] & 0 & 12 [ 8] \\
\hline Undefined & $16(76)[11]$ & $11(85)$ [ 8] & 15 (65) [10] & 17 (33) [12] & 32 (87) [22] & $91[63]$ \\
\hline Uncodable & $1(5)[1]$ & $2(15)[1]$ & $2(9)[1]$ & $9(18)[6]$ & 3 ( 8$)$ [ 2] & 17 [12] \\
\hline Total & [15] & [9] & [16] & [35] & [25] & $\mathrm{N}=145$ \\
\hline
\end{tabular}

Note. Due to rounding, percentages may not add up to totals. 
Because the sphericity and normality assumptions were violated in each analysis, the Huynh-Felt statistic will be reported in lieu of the omnibus F, because it makes an adjustment based on the degree of failed sphericity (Tabachnick \& Fidell, 2001). In order to protect against the increased probability of Type 1 error that results from multiple testing, a Bonferroni adjustment was made to produce a more conservative alpha, and the tests will be considered significant at the .01 (two-tailed) level. Finally, transformations of the data did not yield different conclusions from the untransformed data, so the results are reported using the untransformed data for the purpose of improving interpretation.

\section{Moral Judgment Ratings}

When compared to nonprototypes, it was predicted that immoral prototypes would produce the highest levels of wrong and blame, and promoral prototypes would produce the highest levels of right and praise. In general, it was also predicted that prototypes would be easier to judge than nonprototypes.

Cloning Scenario (Immoral). Using Pillai’s Trace, there was a significant type of condition effect on the three dependent variables: right/wrong, praise/blame, and difficulty, $V=.16, F(12,1428)=6.78, p<.001, \eta_{\mathrm{p}}{ }^{2}=.06$. Separate univariate ANOVA's reveal a significant type of condition effect on the level of right/wrong $F(4,476)=13.00$, $p<.001, \eta_{\mathrm{p}}{ }^{2}=.10$; praise/blame, $F(4,476)=18.50, p<.001, \eta_{\mathrm{p}}{ }^{2}=.14$; and difficulty level, $F(4,476)=7.28, p<.001 ; \eta_{\mathrm{p}}^{2}=.06$

Right/Wrong. Post hoc tests (Games-Howell) show that the immoral cloning prototype condition was rated as more wrong than the intentions, $p=.01, d=-0.50,95 \%$ CI [-0.74, -.07]; consent, $p<.001, d=-0.77$, CI [-1.26, -0.36], and outcomes, $p<.001, d$ $=-0.69$, CI $[-0.89,-0.25]$ conflict conditions (see Table XVII for a listing of means, 
standard deviations, and confidence intervals). The social context conflict was rated as more wrong than the consent, $p<.001, d=-0.72$, CI [-1.22, -0.32] and outcomes, $p<.001, d=-0.64$, CI [ $-0.89,-0.25]$ conflict conditions, while approaching a significant difference when compared to the intentions conflict condition, $p=.03$, $d=-0.45$, CI [0.70, -0.03], ns. 
TABLE XVII

MEAN (SD) [95\% CI] LEVELS OF RIGHT/WRONG, PRAISE/BLAME AND DIFFICULTY BY CONDITION

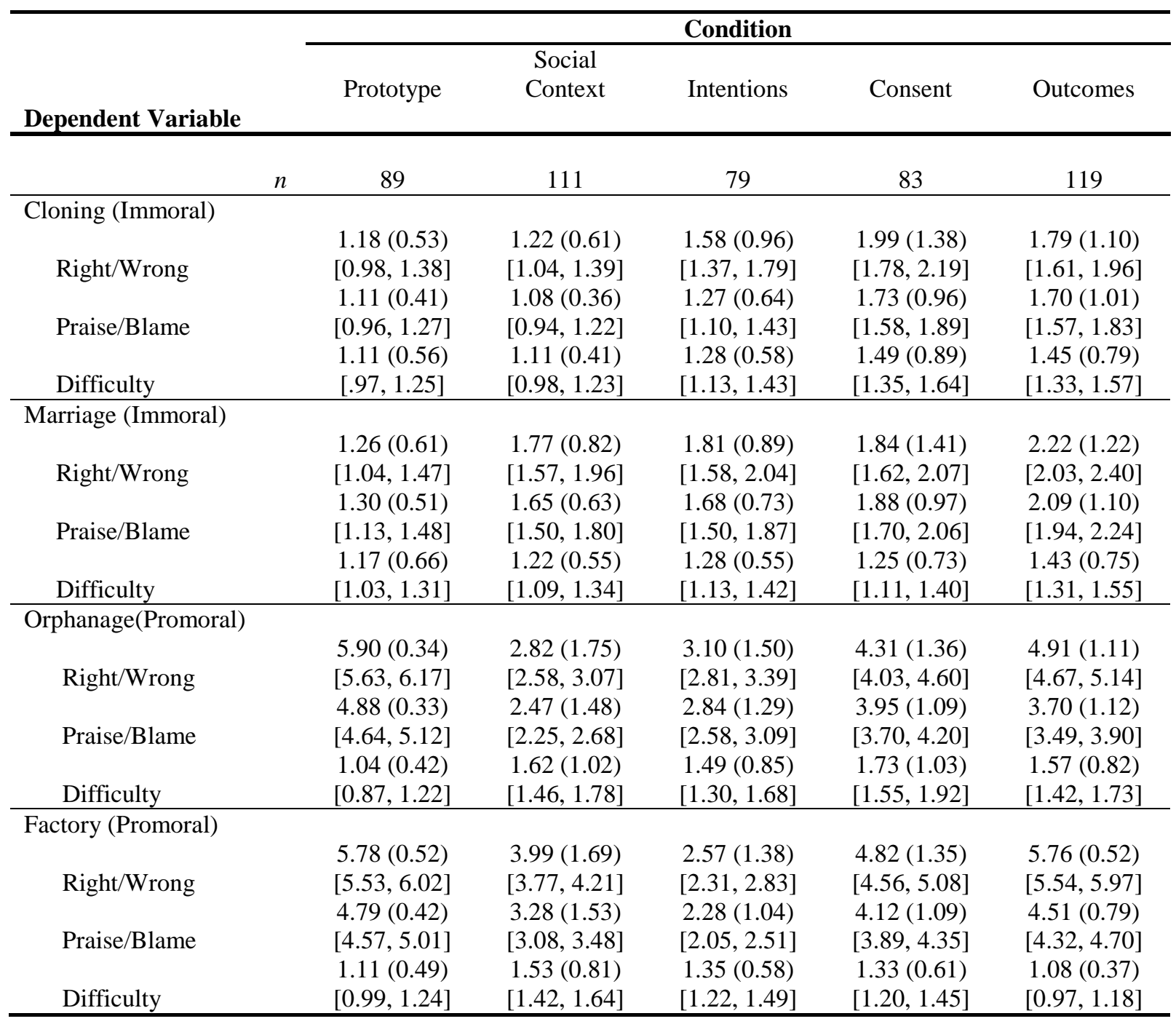

Note. Wrong vs. Right ratings are based on a 6-point, bi-polar scale ( 1 = very wrong, 6 = very right).

Praise vs. Blame ratings are based on a 5-point, bi-polar scale ( 1 = full blame, $3=$ no praise or blame, $5=$ full praise). Difficulty level ratings are based on a 5-point scale ( 1 = not difficult, 5 = extremely difficult). 
Praise/Blame. When asked to assign praise/blame to the actors in the cloning scenario, the participants felt that the actors in the immoral prototype condition deserved more blame than in the consent, $p=<.001, d=-0.84$, CI $[-0.94,-0.31]$ or outcomes, $p=$ $<.001, d=-0.77, \mathrm{CI}[-0.87,-0.30]$ conflict conditions. Likewise, the actors in the social context conflict condition received more blame than the actors in the consent, $p<.001, d$ $=-0.90$, CI [-0.96, -0.34] and outcomes, $p<.001, d=-0.82$, CI [-0.89, -0.35$]$ conflict conditions; and the actors in the intentions conflict condition received more blame than the consent, $p=.003, d=-0.56$, CI [-0.82, -0.12$]$ and outcomes, $p=.003, d=-0.51$, CI [0.75, -0.11] conflict conditions.

Difficulty. When asked to rate the difficulty level of making a moral judgement, the hypothetical in the immoral prototype condition was easier to judge than those in the consent, $p=.009, d=-0.51$, CI [-0.70, -0.07] and outcomes, $p=.004, d=-0.50$, CI [ 0.59, -0.08] conflict conditions. In addition, the hypothetical in the social context conflict condition was easier to judge than the ones in the consent, $p=.003, d=-0.55$, CI [-0.68, 0.09] and outcomes, $p=.001, d=-0.79$, CI [ $-0.56,-0.11]$ conflict conditions.

Marriage Scenario (Immoral). Using Pillai’s Trace, there was a significant type of condition effect on the three dependent variables: right/wrong, praise/blame, and difficulty, $V=.12, F(12,1428)=4.90, p<.001, \eta_{\mathrm{p}}{ }^{2}=.04$. Separate univariate ANOVA's reveal a significant type of condition effect on the level of right/wrong, $F(4,476)=$ 11.81, $p<.001, \eta_{\mathrm{p}}{ }^{2}=.09$ and praise/blame, $F(4,476)=12.61, p<.001, \eta_{\mathrm{p}}{ }^{2}=.10$. However, difficulty level only approached statistical significance, $F(4,476)=2.45, p=$ $.05 ; \eta_{\mathrm{p}}{ }^{2}=.02$, ns, so no post hoc tests were conducted on this dependent variable. 
Right/Wrong. Post hoc tests (Games-Howell) show that the marriage immoral prototype condition was rated as more wrong than the social context, $p<.001, d=-0.71$, 95\% CI [-0.79, -0.23], intentions, $p<.001, d=-0.72$, CI [-0.88, -0.22$]$; consent, $p=.006$, $d=-0.53$, CI [-1.05, -0.12], and outcomes, $p<.001, d=-1.00$, CI [-1.32, -0.62$]$ conflict conditions. The social context conflict was rated as more wrong than the outcomes $p=$ $.009, d=-0.43$, CI $[-0.83,-0.08]$ conflict condition. The difference between the intentions and outcomes conflict conditions approached statistical significance $p=.05, d$ $=-0.38$, CI $[-0.82,0.00]$, ns.

Praise/Blame. When it came to assigning praise/blame, the immoral prototype condition received more blame than the social context, $p<.001, d=-0.61$, CI [-0.57, $0.12]$; intentions, $p=.001, d=-0.60$, CI [-0.65, -0.11$]$; consent, $p<.001, d=-0.75$, CI [$0.91,-0.25]$ and outcomes, $p<.001, d=-0.92$, CI [-1.10, -0.47] conflict conditions. Additionally, the social context conflict condition received more blame than the outcomes conflict condition, $p=.002, d=-0.49$, CI [-0.77, -0.12]. The difference between the intentions and outcomes conflict conditions approached statistical signficance, $p=.02, d=-0.44$, CI [-0.77, -0.05], ns.

Orphanage Scenario (Promoral). Using Pillai’s Trace, there was a significant type of condition effect of type on the three dependent variables: right/wrong, praise/blame, and difficulty, $V=.58, F(12,1428)=28.61, p<.001, \eta_{\mathrm{p}}{ }^{2}=.19$. Separate univariate ANOVA's reveal a significant effect of type of condition on the level of right/wrong, $F(4,476)=90.65, p<.001, \eta_{p}{ }^{2}=.43$, praise/blame, $F(4,476)=65.02, p<$ $.001, \eta_{\mathrm{p}}{ }^{2}=.35$, and difficulty level, $F(4,476)=8.57, p<.001 ; \eta_{\mathrm{p}}{ }^{2}=.07$ 
Right/Wrong. Post hoc tests (Games-Howell) show that the promoral orphanage prototype condition was rated as more right than the conditions with outcomes, $p<.001$, $d=1.21$, CI [0.69, 1.29], consent, $p<.001, d=1.60$, CI [1.16, 2.01], intentions $p<.001$, $d=2.57$, CI [2.32, 3.28], and social context, $p<.001, d=2.44$, CI [2.61, 3.55] conflict conditions. The outcomes conflict was rated as more right than the consent, $p=.01, d=$ 0.23, CI [0.10, 1.09], intentions, $p<.001, d=1.37, \mathrm{CI}[1.26,2.35]$, and social context, $p$ $<.001, d=1.43$, CI $[1.55,2.62]$ conflict conditions. The consent conflict condition was rated as more right than the intentions, $p<.001, d=0.85$, CI $[0.59,1.83]$ and social context, $p<.001, d=0.95$, CI [ $0.88,2.11]$ conflict conditions.

Praise/Blame. When participants had to assign levels of praise or blame, the promoral prototype condition received more praise than the consent, $p<.001, d=0.68$, CI [0.58, 1.27], outcomes, $p<.001, d=1.42$, CI [0.88, 1.48], intentions, $p<.001, d=$ 2.17, CI [1.63, 2.46] and social context $p<.001, d=2.25$, CI [2.01, 2.81] conflict conditions. The consent conflict condition received more praise than the immoral intentions, $p<.001, d=0.93$, CI [0.60, 1.63], and social context, $p<.001, d=1.14$, CI [ 0.97, 1.99] conflict conditions. Likewise, the participants in the outcomes condition also assigned more praise than the participants in the intentions, $p<.001, d=0.71$, CI [0.37, $1.35]$ and social context, $p<.001, d=0.94$, CI $[0.75,1.71]$ conflict conditions.

Difficulty. When the participants were asked to rate the difficulty level in making a moral judgment, the promoral prototype condition was rated as easier to judge than the intentions, $p<.001, d=-0.67$, CI [-0.74, -0.16$]$, outcomes, $p<.001, d=-0.81$, CI[-0.77, -0.29], social context, $p<.001, d=-0.74$, CI [-0.87, -0.28 ], and consent, $p<.001, d=$ 0.88, CI[ -1.03, -0.35] conflict conditions. 
Factory Scenario (Promoral). Using Pillai’s Trace, there was a significant type of condition effect on the three dependent variables: right/wrong, praise/blame, and difficulty, $V=.57, F(12,1428)=28.61, p<.001, \eta_{\mathrm{p}}^{2}=.19$. Separate univariate ANOVA's reveal a significant effect of type of condition on the level of right/wrong, $F(4,476)=115.90, p<.001, \eta_{\mathrm{p}}{ }^{2}=.49$, praise/blame, $F(4,476)=82.90, p<.001, \eta_{\mathrm{p}}{ }^{2}=$ .41 , and difficulty level, $F(4,476)=10.88, p<.001 ; \eta_{\mathrm{p}}^{2}=.08$.

Right/Wrong. Post hoc tests (Games-Howell) show that the factory work promoral prototype condition was rated as more right than the conditions with consent, $p$ $<.001, d=0.94$, CI [0.52, 1.40], social context, $p<.001, d=1.43$, CI [1.32, 2.25], and intentions, $p<.001, d=3.08$, CI $[2.75,3.66]$ conflict conditions. The outcomes condition was also rated as more right than the consent, $p<.001, d=0.92$, CI $[0.50$, 1.37], social context, $p<.001, d=1.42$, CI[1.30, 2.23], and intentions, $p<.001, d=3.06$, CI $[2.73,3.64]$ conflict conditions. The consent conflict condition was rated as more right than the immoral social context, $p=.002, d=0.54$, CI[0.23, 1.43], and intentions, $p<$ $.001, d=1.65, \mathrm{CI}[1.66,2.84]$ conflict conditions. The social context condition was rated as more right than the intentions conflict condition, $p<.001, d=0.92$, CI[0.81, 2.04].

Praise/Blame. When it came to assigning praise/blame, the promoral prototype condition received more praise than the outcomes, $p=.01, d=0.44, \mathrm{CI}[0.04,0.51]$, consent, $p<.001, d=0.81$, CI[0.31, 1.02], social context, $p<.001, d=1.35$, CI[1.09, 1.93], and intentions, $p<.001, d=3.16$, CI[2.16, 2.85] conflict conditions. The outcomes conflict condition received more praise than the social context, $p<.001, d=$ 1.01, $\mathrm{CI}[0.79,1.68]$, and intentions, $p<.001, d=2.14, \mathrm{CI}[1.85,2.61]$ conflict conditions, and it approached a significant difference when compared to consent, $p<.04, d=0.41$, 
CI[.01, .78], ns. The consent conflict condition received more praise than the social context, $p<.001, d=0.63$, CI[0.32, 1.36] and intentions, $p<.001, d=1.73$, CI $[1.38$, 2.30] conflict conditions. The social context conflict condition received more praise than the intentions conflict condition, $p<.001, d=0.76$, CI[0.49, 1.51].

Difficulty. When participants were asked to rate the difficulty level of making a moral judgment, the promoral prototype condition was easier to judge than the social context conflict condition, $p<.001, d=-0.63$, CI[-0.67, -0.16$]$ and approached a significant difference when compared to the intentions conflict condition, $p=.03, d=$ 0.45 , CI[-0.47, -0.01$], n s$. The outcomes conflict condition was easier to judge than the consent, $p=.01, d=-0.50$, CI[-0.46, -0.04$]$, intentions, $p=.002, d=-0.56$, CI[-0.48, $0.08]$, and social context, $p<.001, d=-0.71$, CI[-0.69, -0.22$]$ conflict conditions.

Overall, none of the nonprototypes were rated as more wrong and blameworthy than the immoral prototypes or more right and praiseworthy than the promoral prototypes, and the prototypes significantly differed from most of the nonprototypes in their respective scenario sets. This suggests that prototypes tend to elicit extreme judgments. However, there was no clear, consistent pattern that discriminated between prototypes and nonprototypes when it came to rating the difficulty level of making a judgment.

\section{Moral Judgment Frequencies and Percentages}

It was predicted that moral prototypes would elicit perfect or near perfect agreement. In the immoral prototype conditions (i.e., the cloning and marriage prototypes), 88 out of 89 participants judged these scenarios as wrong (99\%). In the promoral prototype conditions (i.e., the factory work and orphanage prototypes), all 89 
(100\%) participants judged these scenarios as right. Overall, the immoral marriage and cloning nonprototype conditions produced more "wrong” than "right” judgments, ranging between $82 \%$ - 98\% agreement that the actions in the scenarios were wrong. In the promoral factory work and orphanage nonprototype condtions, the percentage of participants rating these scenarios as "right” ranged from 24\% - 99\% agreement. (See Table XVIII for a listing of sample sizes, frequencies, and percentages.) These findings show that moral prototypes produce a high percentage of agreement while ratings about nonprototypical situations appear to be more divided.

TABLE XVIII

FREQUENCY AND (PERCENTAGE) OF WRONG AND RIGHT RESPONSES

\begin{tabular}{|c|c|c|c|c|c|c|c|c|c|c|c|}
\hline \multirow[b]{2}{*}{ Scenario } & & \multicolumn{10}{|c|}{ Condition } \\
\hline & & \multicolumn{2}{|c|}{$\begin{array}{c}\text { Social } \\
\text { Context } \\
\end{array}$} & \multicolumn{2}{|c|}{ Intentions } & \multicolumn{2}{|c|}{ Consent } & \multicolumn{2}{|c|}{ Outcomes } & \multicolumn{2}{|c|}{ Prototype } \\
\hline & $n$ & \multicolumn{2}{|c|}{111} & \multicolumn{2}{|c|}{79} & \multicolumn{2}{|c|}{83} & \multicolumn{2}{|c|}{119} & \multicolumn{2}{|c|}{89} \\
\hline Immoral & & W & $\mathrm{R}$ & $\mathrm{W}$ & $\mathrm{R}$ & W & $\mathrm{R}$ & W & $\mathrm{R}$ & W & $\mathrm{R}$ \\
\hline Cloning & & $\begin{array}{l}109 \\
(98)\end{array}$ & $\begin{array}{c}2 \\
(2)\end{array}$ & $\begin{array}{l}77 \\
(98)\end{array}$ & $\begin{array}{c}2 \\
(3)\end{array}$ & $\begin{array}{c}68 \\
(82)\end{array}$ & $\begin{array}{c}15 \\
(18)\end{array}$ & $\begin{array}{l}109 \\
(92)\end{array}$ & $\begin{array}{l}10 \\
(8)\end{array}$ & $\begin{array}{l}88 \\
(99)\end{array}$ & $\begin{array}{c}1 \\
(1)\end{array}$ \\
\hline Marriage & & $\begin{array}{l}108 \\
(97)\end{array}$ & $\begin{array}{c}3 \\
(3)\end{array}$ & $\begin{array}{l}74 \\
(94)\end{array}$ & $\begin{array}{c}5 \\
(6)\end{array}$ & $\begin{array}{c}73 \\
(88)\end{array}$ & $\begin{array}{c}10 \\
(12)\end{array}$ & $\begin{array}{l}105 \\
(88)\end{array}$ & $\begin{array}{c}14 \\
(12)\end{array}$ & $\begin{array}{c}88 \\
(99)\end{array}$ & $\begin{array}{c}1 \\
(1)\end{array}$ \\
\hline $\begin{array}{l}\text { Promoral } \\
\text { Factory Work }\end{array}$ & & $\begin{array}{c}44 \\
(40)\end{array}$ & $\begin{array}{c}67 \\
(60)\end{array}$ & $\begin{array}{c}60 \\
(76)\end{array}$ & $\begin{array}{c}19 \\
(24)\end{array}$ & $\begin{array}{c}12 \\
(15)\end{array}$ & $\begin{array}{c}71 \\
(86)\end{array}$ & $\begin{array}{c}1 \\
(1)\end{array}$ & $\begin{array}{l}118 \\
(99)\end{array}$ & $\begin{array}{c}0 \\
(0)\end{array}$ & $\begin{array}{c}89 \\
(100)\end{array}$ \\
\hline Orphanage & & $\begin{array}{c}73 \\
(66)\end{array}$ & $\begin{array}{c}38 \\
(34)\end{array}$ & $\begin{array}{c}49 \\
(62)\end{array}$ & $\begin{array}{c}30 \\
(38)\end{array}$ & $\begin{array}{c}23 \\
(28)\end{array}$ & $\begin{array}{c}60 \\
(72)\end{array}$ & $\begin{array}{c}7 \\
(6)\end{array}$ & $\begin{array}{l}112 \\
(94)\end{array}$ & $\begin{array}{c}0 \\
(0)\end{array}$ & $\begin{array}{c}89 \\
(100)\end{array}$ \\
\hline
\end{tabular}

Note. Due to rounding, the percentages may not add up to 100 . The 6-pt. moral judgment rating scale $(1=$ very wrong $-6=$ very right) was collapsed into a 2 -pt. scale (1-3 = wrong, 4-6 = right). $\mathrm{W}=$ wrong; $\mathrm{R}=$ right 


\section{Moral Justifications}

As moral properties were manipulated to represent prototypes and nonprototypes, it was predicted that moral property language in justifications would vary according to the orientations of the moral properties.

Cloning Scenario (Immoral). Using a criterion of $\alpha=.01$ (two-tailed), type of condition did not produce a significant main effect. However, moral justifications led to a significant within-subjects main effect, Huynh-Feldt $F(3.14,1494.40)=51.37, \mathrm{p}<$

$.001, \eta_{\mathrm{p}}{ }^{2}=.10$. Holding type of condition constant, pairwise comparisons (Bonferroni) on the five levels of moral justifications indicated that most of the participants' responses fell into the consent category (see Table XIX for significance tests results and a listing of proportional means, standard deviations, and confidence intervals). Thus, concerns about consent occurred more often in the participants' responses than social context, intentions, outcomes, and no property justifications. After consent, no property and intentions justifications occurred more frequently than social context and outcomes justifications. Among the 111 no property cases, the highest percentages of responses were categorized as undefined (68\%). 
TABLE XIX

PAIRWISE COMPARISONS (BONFERRONI) OF MORAL JUSTIFICATIONS IN THE CLONING SCENARIO

\begin{tabular}{|c|c|c|c|c|c|}
\hline & \multicolumn{5}{|c|}{ Condition } \\
\hline & Social Context & Intentions & Consent & Outcomes & No Property \\
\hline $\begin{array}{l}\text { Mean }(S D) \\
{[95 \% \mathrm{CI}]}\end{array}$ & $\begin{array}{l}.08(.24) \\
{[.06, .10]}\end{array}$ & $\begin{array}{l}.18(.33) \\
{[.15, .20]}\end{array}$ & $\begin{array}{c}.40(.45) \\
{[.36, .44]}\end{array}$ & $\begin{array}{l}.11(.26) \\
{[.08, .13]}\end{array}$ & $\begin{array}{c}.23(.42) \\
{[.20, .27]}\end{array}$ \\
\hline $\begin{array}{l}\text { Moral } \\
\text { Justifications } \\
\text { Social Context }\end{array}$ & & & & & \\
\hline $\begin{array}{r}p \\
\text { Cohen's } d \\
95 \% \text { CI }\end{array}$ & --- & $\begin{array}{c}<.001^{*} \\
-0.35 \\
{[-.15,-.04]}\end{array}$ & $\begin{array}{c}<.001^{*} \\
-0.89 \\
{[-.39,-.25]}\end{array}$ & $\begin{array}{c}1.00 \\
-0.12 \\
{[-.07, .02]}\end{array}$ & $\begin{array}{c}<.001^{*} \\
-0.44 \\
{[-.22,-.09]}\end{array}$ \\
\hline $\begin{array}{l}\text { Intentions } \\
\qquad \begin{array}{r}\mathrm{p} \\
\text { Cohen’s } d \\
\mathrm{CI}\end{array}\end{array}$ & --- & --- & $\begin{array}{c}<.001 * \\
-0.56 \\
{[-.30,-.14]}\end{array}$ & $\begin{array}{c}.006^{*} \\
0.23 \\
{[.01, .13]}\end{array}$ & $\begin{array}{c}.33 \\
-0.13 \\
{[-0.14,0.02]}\end{array}$ \\
\hline $\begin{array}{l}\text { Consent } \\
\qquad \begin{array}{r}p \\
\text { Cohen’s } d \\
\text { CI }\end{array}\end{array}$ & --- & --- & --- & $\begin{array}{c}<.001^{*} \\
0.79 \\
{[.22, .36]}\end{array}$ & $\begin{array}{c}<.001^{*} \\
0.39 \\
{[.07, .26]}\end{array}$ \\
\hline $\begin{array}{l}\text { Outcomes } \\
\qquad \begin{array}{r}\mathrm{p} \\
\text { Cohen’s } d \\
\mathrm{CI}\end{array}\end{array}$ & --- & --- & --- & --- & $\begin{array}{c}<.001^{*} \\
-0.34 \\
{[-.20,-.06]}\end{array}$ \\
\hline $\begin{array}{r}\text { No Property } \\
\text { pohen's } d \\
\text { CI } \\
\end{array}$ & --- & --- & --- & --- & --- \\
\hline
\end{tabular}

Note. *The mean difference is significant at the .01 level. 
In addition to the moral justification main effect, there was a significant interaction between condition and moral justification, Huynh-Feldt $F(12.56,1494.40)=$ 7.86, $p<.001 ; \eta_{\mathrm{p}}^{2}=.06$. This interaction suggests that the type of condition had a bearing on the participants' moral justifications (see Table XX for a listing of means, standard deviations, and confidence intervals by cell). 
TABLE XX

PROPORTIONAL MEANS (SD) [95\% CI] BY CELL FOR EACH SCENARIO

\begin{tabular}{|c|c|c|c|c|c|}
\hline \multirow[b]{2}{*}{$\begin{array}{c}\text { Moral } \\
\text { Justifications }\end{array}$} & \multicolumn{5}{|c|}{ Condition } \\
\hline & $\begin{array}{c}\text { Social Context } \\
n=111\end{array}$ & $\begin{array}{c}\text { Intentions } \\
n=79\end{array}$ & $\begin{array}{c}\text { Consent } \\
n=83\end{array}$ & $\begin{array}{c}\text { Outcomes } \\
n=119\end{array}$ & $\begin{array}{c}\text { Prototype } \\
n=89\end{array}$ \\
\hline \multicolumn{6}{|l|}{ Immoral } \\
\hline \multicolumn{6}{|l|}{ Cloning } \\
\hline Social & $.02(.10)$ & $.09(.25)$ & $.16(.36)$ & .07 (.23) & $.06(.22)$ \\
\hline Context & {$[-.03, .06]$} & {$[.04, .15]$} & {$[.11, .21]$} & {$[.03, .11]$} & {$[.01, .11]$} \\
\hline \multirow[t]{2}{*}{ Intentions } & $.17(.35)$ & $.08(.20)$ & $.34(.44)$ & $.16(.30)$ & $.12(.27)$ \\
\hline & {$[.11, .23]$} & {$[.01, .16]$} & {$[.27, .41]$} & {$[.10, .21]$} & {$[.06, .19]$} \\
\hline \multirow[t]{2}{*}{ Consent } & $.47(.47)$ & $.54(.44)$ & $.07(.25)$ & $.49(.46)$ & $.42(.45)$ \\
\hline & {$[.39, .55]$} & {$[.45, .63]$} & {$[-.02, .16]$} & {$[.41, .56]$} & {$[.33, .51]$} \\
\hline \multirow[t]{2}{*}{ Outcomes } & $.09(.24)$ & $.10(.27)$ & $.12(.29)$ & $.14(.26)$ & $.08(.22)$ \\
\hline & {$[.04, .14]$} & {$[.04, .16]$} & {$[.07, .18]$} & {$[.09, .18]$} & {$[.03, .13]$} \\
\hline \multirow[t]{2}{*}{ No Property } & $.25(.44)$ & .18 ( . .38) & $.28(.45)$ & $.15(.36)$ & $.32(.47)$ \\
\hline & {$[.17, .33]$} & {$[.09, .27]$} & {$[.19, .37]$} & {$[.08, .23]$} & {$[.23, .40]$} \\
\hline \multicolumn{6}{|l|}{ Marriage } \\
\hline Social & $.08(.22)$ & $.11(.28)$ & $.22(.35)$ & $.13(.31)$ & $.19(.34)$ \\
\hline Context & {$[.02, .13]$} & {$[.04, .17]$} & {$[.16, .29]$} & {$[.08, .18]$} & {$[.13, .26]$} \\
\hline \multirow[t]{2}{*}{ Intentions } & $.30(.43)$ & $.24(.37)$ & $.43(.44)$ & $.25(.37)$ & $.37(.42)$ \\
\hline & {$[.23, .38]$} & {$[.16, .33]$} & {$[.34, .52]$} & {$[.18, .32]$} & {$[.28, .45]$} \\
\hline \multirow[t]{2}{*}{ Consent } & $.34(.45)$ & $.30(.43)$ & $.12(.30)$ & $.18(.35)$ & $.13(.30)$ \\
\hline & {$[.27, .41]$} & {$[.22, .39]$} & {$[.04, .20]$} & {$[.11, .25]$} & {$[.06, .21]$} \\
\hline \multirow[t]{2}{*}{ Outcomes } & .05 (.19) & $.04(.13)$ & $.03(.12)$ & $.22(.36)$ & $.04(.16)$ \\
\hline & {$[.01, .09]$} & {$[-.01, .09]$} & {$[-.01, .08]$} & {$[.18, .26]$} & {$[-.00, .09]$} \\
\hline \multirow[t]{2}{*}{ No Property } & $.24(.43)$ & $.30(.46)$ & $.19(.40)$ & $.22(.41)$ & $.26(.44)$ \\
\hline & {$[.16, .32]$} & {$[.21, .40]$} & {$[.10, .29]$} & {$[.14, .30]$} & {$[.17, .35]$} \\
\hline \multicolumn{6}{|l|}{ Promoral } \\
\hline \multicolumn{6}{|l|}{ Factory Work } \\
\hline Social & $.41(.47)$ & $.00(.00)$ & $.00(.00)$ & $.00(.00)$ & $.00(.00)$ \\
\hline Context & {$[.37, .46]$} & {$[-.05, .05]$} & {$[-.05, .05]$} & {$[-.04, .04]$} & {$[-.05, .05]$} \\
\hline \multirow[t]{2}{*}{ Intentions } & $.10(.26)$ & $.59(.41)$ & $.21(.37)$ & $.23(.38)$ & $.10(.27)$ \\
\hline & {$[.04, .16]$} & {$[.52, .67]$} & {$[.14, .28]$} & {$[.17, .29]$} & {$[.03, .17]$} \\
\hline \multirow[t]{2}{*}{ Consent } & $.01(.07)$ & $.02(.12)$ & $.18(.35)$ & $.02(.11)$ & $.00(.00)$ \\
\hline & {$[-.02, .04]$} & {$[-.02, .05]$} & {$[.15, .22]$} & {$[-.01, .05]$} & {$[-.04, .04]$} \\
\hline \multirow[t]{2}{*}{ Outcomes } & $.19(.37)$ & $.21(.31)$ & $.24(.38)$ & $.36(.45)$ & $.34(.46)$ \\
\hline & {$[.12, .27]$} & {$[.13, .30]$} & {$[.16, .33]$} & {$[.29, .43]$} & {$[.27, .43]$} \\
\hline \multirow[t]{2}{*}{ No Property } & $.28(.45)$ & .18 (.38) & $.35(.48)$ & $.40(.49)$ & $.55(.50)$ \\
\hline & {$[.19, .37]$} & {$[.07, .28]$} & {$[.25, .45]$} & {$[.31, .48]$} & {$[.45, .65]$} \\
\hline \multicolumn{6}{|l|}{ Orphanage } \\
\hline Social & $.59(.46)$ & $.01(.08)$ & $.04(.19)$ & $.04(.17)$ & $.08(.27)$ \\
\hline Context & {$[.53, .64]$} & {$[-.05, .08]$} & {$[-.02, .10]$} & {$[-.01, .09]$} & {$[.03, .14]$} \\
\hline \multirow{2}{*}{ Intentions } & .05 (.18) & $.65(.39)$ & $.12(.29)$ & $.34(.45)$ & $.12(.30)$ \\
\hline & {$[-.02, .11]$} & {$[.57, .72]$} & {$[.05, .19]$} & {$[.28, .40]$} & {$[.05, .19]$} \\
\hline \multirow[t]{2}{*}{ Consent } & $.01(.07)$ & $.00(.00)$ & $.32(.42)$ & $.00(.00)$ & $.01(.05)$ \\
\hline & {$[-.02, .05]$} & {$[-.04, .04]$} & {$[.28, .36]$} & {$[-.03, .03]$} & {$[-.03, .04]$} \\
\hline Outcomes & $.17(.35)$ & $.17(.26)$ & $.24(.39)$ & .18 (.33) & $.37(.46)$ \\
\hline & {$[.10, .23]$} & {$[.09, .25]$} & {$[.16, .32]$} & {$[.11, .24]$} & {$[.30, .45]$} \\
\hline No Property & .19 (.39) & $.16(.37)$ & $.28(.45)$ & $.45(.50)$ & $.53(1.23)$ \\
\hline & {$[.07, .31]$} & {$[.02, .31]$} & {$[.14, .42]$} & {$[.33, .56]$} & {$[.39, .67]$} \\
\hline
\end{tabular}


Intentions. In the intentions conflict condition, which depicts the scientists as motivated by the desire to help others, pairwise comparisons (Bonferroni) show that participants used consent justifications more frequently than the no property, $p<.001, d$ $=0.88,95 \%$ CI $[.13, .60]$, outcomes, $p<.001, d=1.21$, CI $[.25, .63]$, social context, $p<$ $.001, d=1.26$, CI [.26, .63], and intentions, $p<.001, d=1.35$, CI [.29, .62] justifications. Among the 14 no property responses in this condition, the participants tended to provide undefined (71\%) justifications.

Consent. In the consent condition, which depicts people consenting to the scientists’ actions, pairwise comparisons show that intentions justifications appeared more often than outcomes, $p=.007, d=0.59$, CI [.04, .40] and consent, $p<.001, d=$ 0.75 , CI $[.10, .45]$ justifications. In addition, there were more no property justifications than consent, $p=.01, d=.58$, CI $[.03, .38]$. Among the 23 no property responses in this condition, the participants tended to use undefined (73\%) justifications.

Outcomes. In the outcomes conflict condition, which presents positive outcomes as a result of the scientists' actions, consent resulted in the greatest proportional mean and was used significantly more often than no property, $p<.001, d=0.82$, CI [.15, .52]; intentions, $p<.001, \mathrm{~d}=0.85$, CI [.16, .50]; outcomes, $p<.001, d=0.94$, CI [.19, .51]; and social context, $p<.001, d=1.15$, CI [.27, .57] justifications. Among the 18 no property responses in this condition, the participants tended to use undefined (61\%) justifications.

Social Context. In the social context conflict condition, which included an action generally condoned by people in our society, the consent justification received the greatest proportional mean and was used significantly more often than intentions, $p<$ 
$.001, d=0.72$, CI [.11, .49]; outcomes, $p<.001, d=1.01$, CI [.23, .54]; and social context, $p<.001, d=1.32$, CI [.32, 59]. Consent approached statistical significance when compared to no property justifications, $p=.05, d=0.48$, CI [.-.001, .44], $n s$. No property justifications were used more often than social context justifications, $p<.001, d$ $=0.72$, CI $[.11, .36]$, and it approached statistical significance when compared to outcomes justifications, $p=.02, d=0.45$, CI [.02, .31], ns. The intentions justification was also used more often the social context justification, $p<.001, d=0.58$, CI [.05, .26], while outcomes justifications approached statistical significance when compared to social context justifications, $p=.04, d=0.38$, CI [.001, .15], ns. Among the 28 no property responses in this condition, the participants tended to use undefined (57\%) and authority and norms (25\%) justifications.

Prototype. In the prototype condition, the consent and no property justifications resulted in the greatest proportional means. The consent justification was used more often than intentions, $p<.001, d=0.81$, CI [.12, .47]; outcomes, $p<.001, d=0.96$, CI $[.18, .51]$; and social context, $p<.001, d=1.02$, CI $[.20, .53]$ justifications. The no property justification occurred more frequently than outcomes, $p=.002, d=0.65$, CI $[.06, .41]$ and social context, $p<.001, d=0.71$, CI $[.09, .42]$ justifications, and it approached statistical significance when compared to intentions, $p=.04, d=0.52$, CI $[.01, .38], n s$. Among the 28 no property responses in this condition, the participants often reported undefined (75\%) justifications.

Marriage Scenario (Immoral). Using a criterion of $\alpha=.01$ (two-tailed), type of condition did not produce a significant main effect. However, moral justifications led to a significant within-subjects main effect, Huynh-Feldt $F(3.35,1592.61)=25.43$, p < 
$.001, \eta_{\mathrm{p}}{ }^{2}=.05$. Holding type of condition constant, pairwise comparisons (Bonferroni) on the four levels of moral justifications indicated that most of the participants' responses fell into the intentions and no property categories (see Table XXI for significance tests results and a listing of proportional means, standard deviations, and confidence intervals). The participants used concerns about intentions in their responses more often than consent, social context, and outcomes. The participants’ responses fell into the no property category significantly more often than the social context and outcomes categories. Concerns about consent appeared more often than outcomes justifications and approached statistical significance when compared to social context justifications. Among the 110 no property responses in this condition, the participants tended to use undefined (62\%) justifications. 
TABLE XXI

PAIRWISE COMPARISONS (BONFERRONI) OF MORAL JUSTIFICATIONS

IN THE MARRIAGE SCENARIO

\begin{tabular}{|c|c|c|c|c|c|}
\hline & \multicolumn{5}{|c|}{ Condition } \\
\hline & Social Context & Intentions & Consent & Outcomes & No Property \\
\hline $\begin{array}{l}\text { Mean }(S D) \\
{[95 \% \mathrm{CI}]}\end{array}$ & $\begin{array}{l}.15(.30) \\
{[.12, .17]}\end{array}$ & $\begin{array}{c}.32(.41) \\
{[.28, .36]}\end{array}$ & $\begin{array}{l}.22(.38) \\
{[.18, .25]}\end{array}$ & $\begin{array}{l}.08(.23) \\
{[.06, .10]}\end{array}$ & $\begin{array}{l}.24(.43) \\
{[.20, .28]}\end{array}$ \\
\hline $\begin{array}{l}\text { Moral } \\
\text { Justifications } \\
\text { Social Context }\end{array}$ & & & & & \\
\hline $\begin{array}{r}p \\
\text { Cohen's } d \\
95 \% \text { CI }\end{array}$ & --- & $\begin{array}{c}<.001^{*} \\
-0.47 \\
{[-.25,-.10]}\end{array}$ & $\begin{array}{c}.05 \\
-0.20 \\
{[-.14, .00]}\end{array}$ & $\begin{array}{c}.002 * \\
0.26 \\
{[.02, .12]}\end{array}$ & $\begin{array}{c}.003 * \\
-0.24 \\
{[-.17,-.02]}\end{array}$ \\
\hline $\begin{array}{l}\text { Intentions } \\
\qquad \begin{array}{r}\mathrm{p} \\
\text { Cohen’s } d \\
\mathrm{CI}\end{array}\end{array}$ & --- & --- & $\begin{array}{c}.005 * \\
.34 \\
{[.02, .19]}\end{array}$ & $\begin{array}{c}<.001^{*} \\
0.34 \\
{[.18, .30]}\end{array}$ & $\begin{array}{c}.21 \\
0.19 \\
{[-.02, .17]}\end{array}$ \\
\hline \begin{tabular}{l} 
Consent \\
\multicolumn{1}{c}{$p$} \\
Cohen's $d$ \\
CI
\end{tabular} & --- & --- & --- & $\begin{array}{c}<.001^{*} \\
0.45 \\
{[.08, .20]}\end{array}$ & $\begin{array}{c}1.00 \\
-0.05 \\
{[-.11, .06]}\end{array}$ \\
\hline $\begin{array}{l}\text { Outcomes } \\
\text { Cohen's } d \\
\mathrm{CI}\end{array}$ & --- & --- & --- & --- & $\begin{array}{c}<.001 * \\
-0.46 \\
{[-.23,-.10]}\end{array}$ \\
\hline $\begin{array}{r}\text { No Property } \\
\text { p } \\
\text { Cohen’s } d \\
\text { CI }\end{array}$ & --- & --- & --- & --- & --- \\
\hline
\end{tabular}

Note. *The mean difference is significant at the .01 level. 
In addition to the moral justification main effect, there was a significant interaction between condition and moral justification, Huynh-Feldt $F(13.38,1592.61)=$ 4.27, $\mathrm{p}<.001 ; \eta_{\mathrm{p}}{ }^{2}=.04$. This interaction suggests that the type of condition had a bearing on the participants’ moral justifications (see Table XIX for a listing of means, standard deviations, and confidence intervals by cell).

Intentions. In the intentions conflict condition, which depicted the actor as marrying to help his friend; intentions, consent, and no property justifications were used more often than outcomes justification $p<.001, d=0.72$, CI $[.08, .33]$; $p<.001, d=0.82$, CI $[.11, .42] ; p<.001, d=1.04$, CI $[.10, .43]$. No property and consent justifications approached statistical significance when compared to social context justifications $p=.05$, $d=0.50$, CI $[.001, .39] ; p=.03, d=0.52$, CI $[.01, .38]$. Among the 23 no property responses in this condition, the participants tended to use undefined (44\%) and counterfactual (22\%) justifications.

Consent. In the consent conflict condition, which depicted consent to marry, intentions justifications resulted in the greatest proportional mean and were included in more responses than consent, $p<.001, d=0.82$, CI $[.12, .50]$ and outcomes, $p<.001, d=$ 1.24, CI [.25, .54]. It approached statistical significance when compared to social context, $p=.05, d=0.53$, CI [.002, .42], ns, and no property, $p=.03, d=0.57$, CI [.01, .47], ns, justifications. Social Context and no property justifications were used significantly more often than outcomes justifications, $p<.001, d=0.73$, CI [.07, .30]; $p=$ $.01, d=0.54$, CI $[.02, .30]$. Among the 15 no property responses in this condition, the participants tended to use undefined (60\%), and uncodable (27\%) justifications. 
Outcomes. In the outcomes condition, which presents a positive outcome, there were no significant differences between the justifications ( $p>.13)$. Among the 24 no property responses in this condition, the participants tended to use undefined (58\%) justifications.

Social Context. In the social context conflict condition, which presents a couple in love, the intentions, consent, and no property justifications received the greatest proportional means and were used more often than social context, $p<.001, d=0.64$, CI $[.08, .36] ; p<.001, d=0.73$, CI [.11, .40]; $p=.01, d=0.47$, CI $[.03, .30]$ and outcomes, $p<.001, d=0.75$, CI [.12, .38]; $p<.001, d=0.84$, CI [.15, .42]; $p=.001, d=0.57$, CI $[.06, .33]$ justifications. Among the 34 no property responses in this condition, the participants tended to use undefined (60\%) and counterfactual (22\%) justifications.

Prototype. In the prototype condition, intentions justifications were cited more frequently than consent, $p=.002, d=0.47$, CI [.06, .41] and outcomes, $p<.001, d=$ 1.04 , CI $[.18, .47]$ justifications. The no property and social context justifications occurred more frequently than outcome justifications, $\mathrm{p}=.001, d=0.66$, CI [.06, .36]; $p$ $=.003, d=0.56$, CI $[.04, .26]$. Among the 23 no property responses in this condition, the participants tended to use undefined (87\%) justifications.

Factory Job Scenario (Promoral). Using a criterion of $\alpha=.01$ (two-tailed), type of condition did not produce a significant main effect. However, moral justifications led to a significant within-subjects main effect, Huynh-Feldt $F(2.63,1249.77)=55.35, \mathrm{p}<$ $.001, \eta_{\mathrm{p}}{ }^{2}=.10$. Holding type of condition constant, pairwise comparisons (Bonferroni) on the four levels of moral justifications indicated that most of the participants' responses fell into the (see Table XXII) for significance tests results and a listing of proportional 
means, standard deviations, and confidence intervals). Thus, the participants' justifications fell into the no property category more often than the intentions, social context, and consent categories. The outcomes justification was used significantly more often than social context and consent justifications. Social context justifications approached statistical significance when compared to consent justifications. Among the 170 no property responses in this condition, the participants tended to use undefined (64\%) and principle (23\%) justifications. 


\section{TABLE XXII}

PAIRWISE COMPARISONS (BONFERRONI) OF MORAL JUSTIFICATIONS IN THE FACTORY SCENARIO

\begin{tabular}{|c|c|c|c|c|c|}
\hline & \multicolumn{5}{|c|}{ Condition } \\
\hline & Social Context & Intentions & Consent & Outcomes & No Property \\
\hline $\begin{array}{l}\text { Mean }(S D) \\
{[95 \% \mathrm{CI}]}\end{array}$ & $\begin{array}{l}.08(.28) \\
{[.06, .10]}\end{array}$ & $\begin{array}{l}.25(.38) \\
{[.22, .28]}\end{array}$ & $\begin{array}{l}.05(.18) \\
{[.03, .06]}\end{array}$ & $\begin{array}{l}.28(.40) \\
{[.24, .31]}\end{array}$ & $\begin{array}{l}.35(.48) \\
{[.31, .39]}\end{array}$ \\
\hline $\begin{array}{l}\text { Moral } \\
\text { Justifications } \\
\text { Social Context }\end{array}$ & & & & & \\
\hline $\begin{array}{r}p \\
\text { Cohen’s } d \\
95 \% \mathrm{CI}\end{array}$ & --- & $\begin{array}{c}<.001 * \\
-0.51 \\
{[-.22,-.11]}\end{array}$ & $\begin{array}{c}.05 \\
0.13 \\
{[.00, .07]}\end{array}$ & $\begin{array}{c}<.001^{*} \\
-0.58 \\
{[-.25,-.13]}\end{array}$ & $\begin{array}{c}<.001^{*} \\
-0.69 \\
{[-.34,-.19]}\end{array}$ \\
\hline Intentions & & & & & \\
\hline $\begin{array}{r}\mathrm{p} \\
\text { Cohen's } d \\
\mathrm{CI}\end{array}$ & --- & --- & $\begin{array}{c}<.001^{*} \\
0.67 \\
{[.15, .25]}\end{array}$ & $\begin{array}{c}1.00 \\
-0.08 \\
{[-.10, .05]}\end{array}$ & $\begin{array}{c}.01^{*} \\
-0.23 \\
{[-.19,-02]}\end{array}$ \\
\hline $\begin{array}{l}\text { Consent } \\
\qquad \begin{array}{r}p \\
\text { Cohen’s } d \\
\text { CI }\end{array}\end{array}$ & --- & --- & --- & $\begin{array}{c}<.001^{*} \\
-0.74 \\
{[-.29,-.17]}\end{array}$ & $\begin{array}{c}<.001^{*} \\
-0.83 \\
{[-.37,-.24]}\end{array}$ \\
\hline $\begin{array}{l}\text { Outcomes } \\
\qquad \begin{array}{r}\mathrm{p} \\
\text { Cohen’s } d \\
\mathrm{CI}\end{array}\end{array}$ & --- & --- & --- & --- & $\begin{array}{c}.29 \\
-0.16 \\
{[-.02, .18]}\end{array}$ \\
\hline $\begin{array}{r}\text { No Property } \\
\text { p } \\
\text { Cohen’s } d \\
\text { CI }\end{array}$ & --- & --- & --- & --- & --- \\
\hline
\end{tabular}

Note. *The mean difference is significant at the .01 level. 
In addition to the moral justification main effect, there was a significant interaction between condition and moral justification, Huynh-Feldt $F(10.50,1249.77)=$ 17.33, $\mathrm{p}<.001, \eta_{\mathrm{p}}{ }^{2}=.13$. This interaction suggests that the type of condition had a bearing on the participants' moral justifications (see Table XIX for a listing of means, standard deviations, and confidence intervals by cell).

Intentions. In the intentions conflict condition, which depicted the actor as having malevolent motivations, intentions justifications resulted in the greatest proportional mean and were used more frequently than the no property, $p<.001, d=$ 1.04, CI [.18, .65]; outcomes, $p<.001, d=1.05$, CI [.18, .58]; consent, $p<.001, d=$ 1.89, CI [.43, .72]; and social context $p<.001, d=2.04$, CI [.46, .73] justifications. Outcomes justifications resulted in the second greatest proportional mean and were used more frequently than consent, $p<.001, d=0.81$, CI [.09, .31]; and social context justifications $p<.001, d=0.96$, CI $[.11, .31]$. The no property justifications were used more often than the consent, $p=.008, d=0.57$, CI [.03, .29]; and social context justifications, $p=.001, d=0.67$, CI $[.05, .30]$. Among the 14 no property responses in this condition, the participants tended to use undefined (50\%), uncodable (29\%), and principle (20\%) justifications.

Consent. In the consent condition, which depicted a homeless man as being forced to take a job, there were no significant differences between the intentions, consent, outcomes, and no property justifications, $p>.34$, ns. However, the intentions, consent, outcomes and no property justifications appeared more frequently in responses than social context justifications, $p<.001$ and $d=0.80$, CI [.09, .33]; $d=0.73$, CI [.07, .30]; $d$ $=0.89$, CI [.12, .37]; and $d=1.03$, CI [.20, .50], respectively. Among the 47 no property 
responses in this condition, the participants tended to use undefined (52\%) and principle (31\%) justifications.

Outcomes. In the outcomes conflict condition, which states that the man dies performing factory work; the intentions, no property, and outcomes justifications resulted in the greatest proportional means and were used more often in responses than consent, $p$ $<.001, d=0.75$, CI [.11, .32]; $p<.001, d=1.07$, CI [.24, .51]; $p<.001, d=1.04$, CI $[.22, .47]$ and social context, $p<.001, d=0.86$, CI [.13, .33]; $p<.001, d=1.15$, CI [.27, $.52] ; p<.001, d=1.13$, CI [.24, .48] justifications, respectively. Among the 47 no property responses in this condition, the participants tended to use undefined (75\%) justifications.

Social Context. In the social context conflict condition, which depicts a 5-yearold boy working in the factory, the social context and no properties justifications resulted in the greatest proportional means. Social context was used more often than outcomes, $p$ $=.01, d=0.52$, CI $[.03, .41]$; intentions, $p<.001, d=0.82$, CI $[.15, .47]$; and consent $p<$ $.001, d=1.19$, CI $[.27, .53]$ justifications. The no property justification was used more often than intentions, $p=.01, d=0.49$, CI [.02, .34] and consent, $p<.001, d=0.84$, CI $[.14, .39]$. The intentions and outcomes justifications were both used more often than consent $p=.007, d=0.47$, CI $[.02, .16] ; p<.001, d=0.68$, CI $[.08, .28]$. Among the 31 no property responses in this condition, the participants tended to use undefined (58\%) justifications.

Prototype. In the prototype condition, the no property justification resulted in the greatest proportional mean and was used more often than the intentions, $p<.001$, $d=$ 1.12, CI [.25, .65]; social context, $p<.001, d=1.56$, CI $[.40, .70]$; and consent, $p<.001$, 
$d=1.56$, CI $[.40, .70]$ justifications. The outcomes justification was used more frequently than the intentions, $p=.001, d=0.64$, CI [.07, .42]; social context, $p<.001, d$ $=1.05$, CI [.21, .49]; and consent, $p<.001, d=1.05$, CI [.21, .49]. The intentions justification was used more frequently than the social context, $p=.01, d=0.52$, CI [.02, $.19]$ and consent $p=.01, d=0.52$, CI $[.02, .19]$ justifications. Among the 49 no property responses in this condition, the participants tended to use undefined (67\%) and principle (27\%) justifications.

Orphanage Scenario (Promoral). Using a criterion of $\alpha=.01$ (two-tailed), type of condition did not produce a significant main effect. However, moral justifications led to a significant within-subjects main effect, Huynh-Feldt $F(2.39,1135.61)=23.51$, p $<.001, \eta_{\mathrm{p}}{ }^{2}=.05$. Holding type of condition constant, pairwise comparisons (Bonferroni) on the four levels of moral justifications indicated that most of the participants' responses fell into the no property and intentions categories, which were both more frequently used than the social context and consent categories (see Table XXIII for significance tests results and a listing of proportional means, standard deviations, and confidence intervals). The outcomes justifications were used more often than the consent justifications and approached statistical significance when compared to social context. Social context justifications were used more often than consent justifications. Among the 145 no property responses in this condition, the participants tended to use undefined (63\%) justifications. 
TABLE XXIII

PAIRWISE COMPARISONS (BONFERRONI) OF MORAL JUSTIFICATIONS IN THE ORPHANAGE SCENARIO

\begin{tabular}{|c|c|c|c|c|c|}
\hline & \multicolumn{5}{|c|}{ Condition } \\
\hline & Social Context & Intentions & Consent & Outcomes & No Property \\
\hline $\begin{array}{l}\text { Mean }(S D) \\
{[95 \% \mathrm{CI}]}\end{array}$ & $\begin{array}{l}.15(.36) \\
{[.13, .18]}\end{array}$ & $\begin{array}{l}.25(.40) \\
{[.22, .29]}\end{array}$ & $\begin{array}{l}.07(.22) \\
{[.05, .08]}\end{array}$ & $\begin{array}{l}.23(.37) \\
{[.19, .26]}\end{array}$ & $\begin{array}{l}.32(.67) \\
{[.26, .38]}\end{array}$ \\
\hline $\begin{array}{l}\text { Moral } \\
\text { Justifications } \\
\text { Social Context }\end{array}$ & & & & & \\
\hline $\begin{array}{r}p \\
\text { Cohen's } d \\
95 \% \text { CI }\end{array}$ & --- & $\begin{array}{c}<.001^{*} \\
-0.26 \\
{[-.16,-.04]}\end{array}$ & $\begin{array}{c}<.001^{*} \\
0.27 \\
{[.04, .13]}\end{array}$ & $\begin{array}{c}.03 \\
-0.22 \\
{[-.14,-.01]}\end{array}$ & $\begin{array}{c}<.001^{*} \\
-0.32 \\
{[-.27,-.07]}\end{array}$ \\
\hline $\begin{array}{l}\text { Intentions } \\
\qquad \begin{array}{r}\mathrm{p} \\
\text { Cohen's } d \\
\mathrm{CI}\end{array}\end{array}$ & --- & --- & $\begin{array}{c}<.001^{*} \\
0.56 \\
{[.14, .24]}\end{array}$ & $\begin{array}{c}1.00 \\
0.05 \\
{[-.04, .10]}\end{array}$ & $\begin{array}{c}.85 \\
-0.13 \\
{[-.18,0.42]}\end{array}$ \\
\hline $\begin{array}{l}\text { Consent } \\
\qquad \begin{array}{r}p \\
\text { Cohen's } d \\
\text { CI }\end{array}\end{array}$ & --- & --- & --- & $\begin{array}{c}<.001^{*} \\
-0.53 \\
{[-.21,-.10]}\end{array}$ & $\begin{array}{c}<.001 * \\
-0.50 \\
{[-.35,-.16]}\end{array}$ \\
\hline $\begin{array}{l}\text { Outcomes } \\
\qquad \begin{array}{r}\mathrm{p} \\
\text { Cohen's } d \\
\text { CI }\end{array}\end{array}$ & --- & --- & --- & --- & $\begin{array}{c}.16 \\
-0.17 \\
{[-.21, .02]}\end{array}$ \\
\hline $\begin{array}{r}\text { No Property } \\
\text { p } \\
\text { Cohen's } d \\
\text { CI }\end{array}$ & --- & --- & --- & --- & --- \\
\hline
\end{tabular}

Note. *The mean difference is significant at the .01 level. 
In addition to the moral justification main effect, there was a significant interaction between condition and moral justification, Huynh-Feldt $F(9.54,11.35 .61)=$ 21.72, $p<.001, \eta_{\mathrm{p}}{ }^{2}=.15$. This interaction suggests that the type of condition had a bearing on the participants' moral justifications (see Table XIX for a listing of means, standard deviations, and confidence intervals by cell).

Intentions. In the intentions conflict condition, which depicted the actor as doing charity work for selfish gains, the intentions justification resulted in the greatest proportional mean and was used significantly more often than no property $p<.001, d=$ 1.29, CI [.25, .71]; outcomes, $p<.001, d=1.45$, CI [ .30, .65]; social context, $p<.001, d$ $=2.27$, CI [.50, .77]; and consent $p<.001, d=2.36$, CI [.52, .77]. Both the outcomes and no property justifications were used more often than the social context, $p<.001, d=$ 0.83, CI [.07, .25]; $p=.009, d=0.56$, CI [.02, .28] and consent, $p<.001, d=0.92$, CI $[.09, .26] ; p=.002, d=0.61$, CI $[.04, .29]$ justifications. Among the 13 no property responses in this condition, the participants tended to use undefined (85\%) justifications.

Consent. In the consent conflict condition, which depicted the action as being forced onto the orphans and community; the consent, outcomes, and no property justifications resulted in the greatest proportional means. Consent justifications were used significantly more often than intentions, $p=.01, d=0.55$, CI $[.03, .38]$ and social context, $p<.001, d=0.86$, CI [ .12, .44] justifications. The outcomes and no properties justifications were used significantly more often than social context, $p=.002, d=0.65$, CI [.05, .34], $p=.001, d=0.69$, CI [.07, .40] justifications, respectively. Among the 23 no property responses in this condition, the participants tended to use undefined (65\%) justifications. 
Outcomes. In the outcomes conflict condition, which presents the orphans as being harmed, intentions and no property justifications resulted in the greatest proportional means and were used more often than social context, $p<.001, d=0.88$, CI [.16, .43]; $p<.001, d=1.10$, CI [.26, .55] and consent, $p<.001, d=1.07$, CI [.22, .46]; $p$ $<.001, d=1.27$, CI $[.31, .58]$ justifications. The no property justification was used more often than outcomes justifications, $p=.001, d=0.64$, CI [.08, .46], while the intentions justifications approached statistical differences when compared to outcomes justifications, $p=.06, d=0.41$, CI $[-.01, .32]$, ns. The outcomes justification was used more often than social context, $p=.001, d=0.53$, CI $[.04, .23]$ and consent, $p<.001, d=$ 0.77, CI [.09, .26] justifications. Social context justifications approached statistical significance when compared to consent, $p=.07, d=0.33$, CI [-.002, .09], ns. Among the 51 no property responses in this condition, the participants tended to use undefined (33\%) justifications and counterfactual (26\%) justifications.

Social Context. In the social context conflict condition, which presents the orphanage as auctioning babies to the highest bidders, the social context justification resulted in the greatest proportional mean. It was used more often than no property, $p<$ $.001, d=0.94$, CI [.19, .61]; outcomes, $p<.001, d=1.03$, CI [.23, .61]; intentions, $p<$ $.001, d=1.55$, CI [.40, .69; and consent, $p<.001, d=1.76$, CI $[.45, .70]$ justifications. The no properties justification was used more often than intentions, $p=.01, d=0.46$, CI $[.02, .27]$ and consent, $p<.001, d=0.64$, CI [.07, .29] justifications. The outcomes justification was used more often than consent, $p<.001, d=0.63$, CI $[.06, .26]$ and approached statistical significance when compared to intentions, $p=.02, d=0.43$, CI 
[.01, .23], ns. Among the 21 no property responses in this condition, the participants tended to use undefined (76\%) justifications.

Prototype. In the prototype condition, the no property and outcomes justifications resulted in the greatest proportional means. The no property justification was used more frequently than the consent justification, $p=.001, d=0.60$, CI $[.15, .90]$, and it approached statistical significance when compared to intentions, $p=.05, d=0.46$, CI $[.003, .81], n s$, and social context, $p=.02, d=0.51$, CI [.05, .84], ns. The outcomes justification was used more frequently than the intentions, $p=.002, d=0.64$, CI [.06, .43 ]; social context, $p<.001, d=0.77$, CI [.11, .47], and consent, $p<.001, d=1.10$, CI $[.22, .51]$ justifications. The intentions justification was used more frequently than the consent justification, $p=.01, d=0.51$, CI [.02, .21]. Among the 37 no property responses in this condition, the participants tended to use undefined (87\%) justifications.

The findings in this section show that the structure and content of the scenario had a bearing on which properties were included in moral judgment justifications. In some scenario sets, a predominant property became evident in the moral justification language across several conditions, while justifications of other scenarios mirrored the conflicting property. Contrary to what was anticipated, the conflicting property in a nonprototype condition did not always predict the predominant property used in justifications.

\section{Discussion}

\section{Moral Judgment Ratings}

The participants read scenarios that represented a moral prototype or a nonprototype with a conflicting property. They were asked to make a judgment by rating the scenario's level of right/wrong, praise/blame, and difficulty. 
Right/Wrong. For the most part, the right/wrong findings are consistent with the proposed framework of this study. There were significant differences in the ratings between the prototypes and nonprototypes with two exceptions. In the cloning scenario, the immoral prototype did not differ from the social context conflict condition, which presented the medical scientists as developing a disease-fighting drug as opposed to cloning organs (refer to Tables XXIV - XXVII for structural representations of each condition). This suggests that, despite the promoral social context orientation, the combination of bad intentions, a lack of consent, and bad outcomes carried a significant amount of weight in this scenario, causing it to act like the prototype. In the factory scenario, the promoral prototype did not differ significantly from the bad outcomes conflict condition, which depicted a work-related death. In this case, even though the outcomes were harmful, the promoral social context of giving a homeless man a job, good intentions, and consent led to responses that were similar to the promoral prototype condition. 
TABLE XXIV

STRUCTURE OF IMMORAL CLONING SCENARIO

\begin{tabular}{|c|c|c|c|c|}
\hline CONDITION & Social Context & Intentions & Consent & Outcomes \\
\hline Prototype & $\begin{array}{l}\text { Bad: Medical } \\
\text { Scientists sell } \\
\text { cloned body parts } \\
\text { for a profit. }\end{array}$ & $\begin{array}{l}\text { Bad: They do it } \\
\text { for wealth and } \\
\text { power. }\end{array}$ & $\begin{array}{l}\text { Bad: None of the } \\
\text { patients knew } \\
\text { about the cloning } \\
\text { or consented to the } \\
\text { procedures. }\end{array}$ & $\begin{array}{l}\text { Bad: Results in } \\
\text { deaths. }\end{array}$ \\
\hline $\begin{array}{c}\text { Good Social } \\
\text { Context Conflict }\end{array}$ & $\begin{array}{l}\text { Good: Medical } \\
\text { scientists create a } \\
\text { disease-fighting } \\
\text { drug. }\end{array}$ & $\begin{array}{l}\text { Bad: They do it } \\
\text { for wealth and } \\
\text { power. }\end{array}$ & $\begin{array}{l}\text { Bad: None of the } \\
\text { patients knew } \\
\text { about the cloning } \\
\text { or consented to the } \\
\text { procedures. }\end{array}$ & $\begin{array}{l}\text { Bad: Results in } \\
\text { deaths. }\end{array}$ \\
\hline $\begin{array}{c}\text { Good Intentions } \\
\text { Conflict }\end{array}$ & $\begin{array}{l}\text { Bad: Medical } \\
\text { Scientists sell } \\
\text { cloned body parts } \\
\text { for a profit. }\end{array}$ & $\begin{array}{l}\text { Good: They care } \\
\text { about patients and } \\
\text { believe their work } \\
\text { will save lives. }\end{array}$ & $\begin{array}{l}\text { Bad: None of the } \\
\text { patients know } \\
\text { about the cloning } \\
\text { or consented to the } \\
\text { procedures. }\end{array}$ & $\begin{array}{l}\text { Bad: Results in } \\
\text { deaths. }\end{array}$ \\
\hline $\begin{array}{l}\text { Good Consent } \\
\text { Conflict }\end{array}$ & $\begin{array}{l}\text { Bad: Medical } \\
\text { Scientists sell } \\
\text { cloned body parts } \\
\text { for a profit. }\end{array}$ & $\begin{array}{l}\text { Bad: They do it } \\
\text { for wealth and } \\
\text { power. }\end{array}$ & $\begin{array}{l}\text { Good: The } \\
\text { patients requested } \\
\text { the procedures. } \\
\text { They knew about } \\
\text { the cloning and the } \\
\text { associated risks. }\end{array}$ & $\begin{array}{l}\text { Bad: Results in } \\
\text { deaths. }\end{array}$ \\
\hline $\begin{array}{l}\text { Good Outcomes } \\
\text { Conflict }\end{array}$ & $\begin{array}{l}\text { Bad: Medical } \\
\text { Scientists sell } \\
\text { cloned body parts } \\
\text { for a profit. }\end{array}$ & $\begin{array}{l}\text { Bad: They do it } \\
\text { for wealth and } \\
\text { power. }\end{array}$ & $\begin{array}{l}\text { Bad: None of the } \\
\text { patients knew } \\
\text { about the cloning } \\
\text { or consented to the } \\
\text { procedures. }\end{array}$ & $\begin{array}{l}\text { Good: They saved } \\
\text { lives and increased } \\
\text { the number of vital } \\
\text { organs. }\end{array}$ \\
\hline
\end{tabular}


TABLE XXV

STRUCTURE OF IMMORAL MARRIAGE SCENARIO

\begin{tabular}{|c|l|l|l|l|}
\hline CONDITION & \multicolumn{1}{|c|}{ Social Context } & \multicolumn{1}{|c|}{ Intentions } & \multicolumn{1}{c|}{ Consent } & \multicolumn{1}{c|}{ Outcomes } \\
\hline Prototype & $\begin{array}{l}\text { Bad: Fred } \\
\text { marries someone } \\
\text { he finds } \\
\text { disgusting and } \\
\text { does not love. }\end{array}$ & $\begin{array}{l}\text { Bad: Fred wants } \\
\text { Anne to cook, } \\
\text { clean, and have } \\
\text { his children. }\end{array}$ & $\begin{array}{l}\text { Bad: No consent. } \\
\text { Anne is forced } \\
\text { into the marriage. }\end{array}$ & $\begin{array}{l}\text { Bad: Anne is } \\
\text { miserable as his } \\
\text { wife. }\end{array}$ \\
\hline $\begin{array}{c}\text { Good Social } \\
\text { Context Conflict }\end{array}$ & $\begin{array}{l}\text { Good: Fred } \\
\text { marries someone } \\
\text { he loves and who } \\
\text { loves him back. }\end{array}$ & $\begin{array}{l}\text { Bad: Fred wants } \\
\text { Anne to cook, } \\
\text { clean, and have } \\
\text { his children. }\end{array}$ & $\begin{array}{l}\text { Bad: No consent. } \\
\text { Anne is forced } \\
\text { into the marriage. }\end{array}$ & $\begin{array}{l}\text { Bad: Anne is } \\
\text { miserable as his } \\
\text { wife. }\end{array}$ \\
\hline $\begin{array}{l}\text { Good Intentions } \\
\text { Conflict }\end{array}$ & $\begin{array}{l}\text { Bad: Fred } \\
\text { marries someone } \\
\text { he finds } \\
\text { disgusting and } \\
\text { does not love. }\end{array}$ & $\begin{array}{l}\text { Good: He cares } \\
\text { about Anne as a } \\
\text { friend and doesn't } \\
\text { want her to be } \\
\text { sad. }\end{array}$ & $\begin{array}{l}\text { Bad: No consent. } \\
\text { Anne is forced } \\
\text { into the marriage. }\end{array}$ & $\begin{array}{l}\text { Bad: Anne is } \\
\text { miserable as his } \\
\text { wife. }\end{array}$ \\
\hline $\begin{array}{l}\text { Good Consent } \\
\text { Conflict }\end{array}$ & $\begin{array}{l}\text { Bad: Fred } \\
\text { marries someone } \\
\text { he finds } \\
\text { disgusting and } \\
\text { does not love. }\end{array}$ & $\begin{array}{l}\text { clean, and have } \\
\text { his children. }\end{array}$ & $\begin{array}{l}\text { Good: Anne } \\
\text { liked the offer and } \\
\text { accepted Fred's } \\
\text { offer. }\end{array}$ & $\begin{array}{l}\text { Bad: Anne is } \\
\text { miserable as his } \\
\text { wife. }\end{array}$ \\
\hline $\begin{array}{l}\text { Good Outcomes } \\
\text { Conflict }\end{array}$ & $\begin{array}{l}\text { Bad: Fred } \\
\text { marries someone } \\
\text { he finds } \\
\text { disgusting and } \\
\text { does not love. }\end{array}$ & $\begin{array}{l}\text { Bad: Fred wants } \\
\text { Anne to cook, } \\
\text { clean, and have } \\
\text { his children. }\end{array}$ & $\begin{array}{l}\text { Bad: No consent. } \\
\text { Anne is forced } \\
\text { into the marriage. }\end{array}$ & $\begin{array}{l}\text { Good: Anne and } \\
\text { Fred became a } \\
\text { happily married } \\
\text { couple. }\end{array}$ \\
\hline
\end{tabular}


TABLE XXVI

STRUCTURE OF PROMORAL FACTORY SCENARIO

\begin{tabular}{|c|l|l|l|l|}
\hline CONDITION & \multicolumn{1}{|c|}{ Social Context } & \multicolumn{1}{|c|}{ Intentions } & \multicolumn{1}{c|}{ Consent } & \multicolumn{1}{c|}{ Outcomes } \\
\hline Prototype & $\begin{array}{l}\text { Good: Sam offers } \\
\text { a homeless man a } \\
\text { job. }\end{array}$ & $\begin{array}{l}\text { Good: He cares } \\
\text { about people and } \\
\text { wants to help } \\
\text { those in need. }\end{array}$ & $\begin{array}{l}\text { Good: The man } \\
\text { accepts the job. }\end{array}$ & $\begin{array}{l}\text { Good: The man } \\
\text { earns an excellent } \\
\text { salary and lives a } \\
\text { happy, healthy } \\
\text { life. }\end{array}$ \\
\hline $\begin{array}{c}\text { Bad Social } \\
\text { Context Conflict }\end{array}$ & $\begin{array}{l}\text { Bad: Sam offers a } \\
\text { homeless 5-year- } \\
\text { old boy a job. }\end{array}$ & $\begin{array}{l}\text { Good: He cares } \\
\text { about people and } \\
\text { wants to help } \\
\text { those in need. }\end{array}$ & $\begin{array}{l}\text { Good: The man } \\
\text { accepts the job. } \\
\text { earns an excellent } \\
\text { salary and lives a } \\
\text { happy, healthy } \\
\text { life. }\end{array}$ \\
\hline $\begin{array}{c}\text { Bad Intentions } \\
\text { Conflict }\end{array}$ & $\begin{array}{l}\text { Good: Sam offers } \\
\text { a homeless man a } \\
\text { job. }\end{array}$ & $\begin{array}{l}\text { Bad: Sam hates } \\
\text { homeless people } \\
\text { and hopes that the } \\
\text { man will suffer. }\end{array}$ & $\begin{array}{l}\text { Good: The man } \\
\text { accepts the job. }\end{array}$ & $\begin{array}{l}\text { Good: The man } \\
\text { earns an excellent } \\
\text { salary and lives a } \\
\text { happy, healthy } \\
\text { life. }\end{array}$ \\
\hline $\begin{array}{c}\text { Bad Consent } \\
\text { Conflict }\end{array}$ & $\begin{array}{l}\text { Good: Sam offers } \\
\text { a homeless man a } \\
\text { job. }\end{array}$ & $\begin{array}{l}\text { Good: He cares } \\
\text { about people and } \\
\text { wants to help } \\
\text { those in need. }\end{array}$ & $\begin{array}{l}\text { Bad: The man did } \\
\text { not want the job } \\
\text { and was coerced } \\
\text { into working in the } \\
\text { factory. }\end{array}$ & $\begin{array}{l}\text { Good: The man } \\
\text { earns an excellent } \\
\text { salary and lives a } \\
\text { happy, healthy } \\
\text { life. }\end{array}$ \\
\hline $\begin{array}{c}\text { Bad Outcomes } \\
\text { Conflict }\end{array}$ & $\begin{array}{l}\text { Good: Sam offers } \\
\text { a homeless man a } \\
\text { job. }\end{array}$ & $\begin{array}{l}\text { Good: He cares } \\
\text { about people and } \\
\text { wants to help } \\
\text { those in need. }\end{array}$ & $\begin{array}{l}\text { Good: The man } \\
\text { accepts the job. }\end{array}$ & $\begin{array}{l}\text { Bad: The man } \\
\text { died in a factory } \\
\text { accident while } \\
\text { working. }\end{array}$ \\
\hline
\end{tabular}


TABLE XXVII

STRUCTURE OF PROMORAL ORPHANAGE SCENARIO

\begin{tabular}{|c|c|c|c|c|}
\hline CONDITION & Social Context & Intentions & Consent & Outcomes \\
\hline Prototype & $\begin{array}{l}\text { Good: Beth } \\
\text { collects donations } \\
\text { to create a } \\
\text { foundation that } \\
\text { helps orphans find } \\
\text { good homes. }\end{array}$ & $\begin{array}{l}\text { Good: She aims to } \\
\text { be a devoted } \\
\text { advocate for } \\
\text { orphaned children. }\end{array}$ & $\begin{array}{l}\text { Good: The } \\
\text { community and } \\
\text { children value her } \\
\text { contributions and } \\
\text { assistance. }\end{array}$ & $\begin{array}{l}\text { Good: The } \\
\text { children find new } \\
\text { families and live } \\
\text { happy lives. }\end{array}$ \\
\hline $\begin{array}{c}\text { Bad Social } \\
\text { Context Conflict }\end{array}$ & $\begin{array}{l}\text { Bad: Beth raises } \\
\text { money for a } \\
\text { foundation that } \\
\text { helps orphans by } \\
\text { selling the orphans } \\
\text { to the highest } \\
\text { bidders. }\end{array}$ & $\begin{array}{l}\text { Good: She aims to } \\
\text { be a devoted } \\
\text { advocate for } \\
\text { orphaned children. }\end{array}$ & $\begin{array}{l}\text { Good: The } \\
\text { community and } \\
\text { children value her } \\
\text { contributions and } \\
\text { assistance. }\end{array}$ & $\begin{array}{l}\text { Good: The } \\
\text { children find new } \\
\text { families and live } \\
\text { happy lives. }\end{array}$ \\
\hline $\begin{array}{l}\text { Bad Intentions } \\
\text { Conflict }\end{array}$ & $\begin{array}{l}\text { Good: Beth } \\
\text { collects donations } \\
\text { to create a } \\
\text { foundation that } \\
\text { helps orphans find } \\
\text { good homes. }\end{array}$ & $\begin{array}{l}\text { Bad: Beth hates } \\
\text { orphans and only } \\
\text { helps them to get } \\
\text { elected into public } \\
\text { office. }\end{array}$ & $\begin{array}{l}\text { Good: The } \\
\text { community and } \\
\text { children value her } \\
\text { contributions and } \\
\text { assistance. }\end{array}$ & $\begin{array}{l}\text { Good: The } \\
\text { children find new } \\
\text { families and live } \\
\text { happy lives. }\end{array}$ \\
\hline $\begin{array}{c}\text { Bad Consent } \\
\text { Conflict }\end{array}$ & $\begin{array}{l}\text { Good: Beth } \\
\text { collects donations } \\
\text { to create a } \\
\text { foundation that } \\
\text { helps orphans find } \\
\text { good homes. }\end{array}$ & $\begin{array}{l}\text { Good: She aims to } \\
\text { be a devoted } \\
\text { advocate for } \\
\text { orphaned children. }\end{array}$ & $\begin{array}{l}\text { Bad: The } \\
\text { community and } \\
\text { children do not } \\
\text { want her services. } \\
\text { They want to } \\
\text { develop their own } \\
\text { solutions to the } \\
\text { problem. }\end{array}$ & $\begin{array}{l}\text { Good: The } \\
\text { children find new } \\
\text { families and live } \\
\text { happy lives. }\end{array}$ \\
\hline $\begin{array}{l}\text { Bad Outcomes } \\
\text { Conflict }\end{array}$ & $\begin{array}{l}\text { Good: Beth } \\
\text { collects donations } \\
\text { to create a } \\
\text { foundation that } \\
\text { helps orphans find } \\
\text { good homes. }\end{array}$ & $\begin{array}{l}\text { Good: She aims to } \\
\text { be a devoted } \\
\text { advocate for } \\
\text { orphaned children. }\end{array}$ & $\begin{array}{l}\text { Good: The } \\
\text { community and } \\
\text { children value her } \\
\text { contributions and } \\
\text { assistance. }\end{array}$ & $\begin{array}{l}\text { Bad: Some } \\
\text { orphans had been } \\
\text { psychologically } \\
\text { and physically } \\
\text { abused after the } \\
\text { adoptions. }\end{array}$ \\
\hline
\end{tabular}


The close proximity of these two nonprototypical situations to their respective prototypes may have activated a prototypical response. Prototype theory suggests that when stimuli are compared to the prototype and the features are highly correlative, they are likely to be classified as a member of that category (see Churchland, 1998 for discussion). Referring back to the cloning scenario, the combination of bad intentions, lack of consent, and bad outcomes may have closely resembled the immoral prototype. In fact, Turiel et al., (1991) have speculated that these properties have a particularly strong bearing on moral judgments and state that issues prototypical to the moral domain are "intentional... acts with consequences for others..., and the victim is an unwilling participant” (p. 5). Although this combination acted like the prototype in the cloning scenario, the marriage scenario set did not produce the same results. The participants rated the social context condition in the marriage scenario as less wrong than the immoral prototype.

One reason why the social context condition may have differed from the prototype in one scenario set and not the other may be due to the varying magnitudes of the individual properties. For example, the social context property of marrying someone you love might have more promoral power on judgments than creating a disease-fighting drug. Additionally, the magnitude of the outcomes was different in these two conditions. In the marriage scenario, the wife is miserable after she marries. In contrast, the scientists' actions result in deaths. Previous research has produced evidence to support the idea that some judgments are affected by the magnitude of moral properties. For example, Lagnado and Channon (2008) found that intentional actions were rated as more 
blameworthy than unintentional actions when they resulted in the same outcomes. Thus, the level of intentionality had an impact on judgment. In their study, Rai and Holyoak (2010) reexamined the famous trolley dilemma, which asks whether it is okay to sacrifice the life of one person in order to save the lives of five others. Rai and Holyoak created their own version of the dilemma and presented two groups with a different plan to save the passengers. Group 1 was asked to rate its level of agreement with sacrificing two people to save 8 out of 10 passengers, and Group 2 was asked to rate its level of agreement with sacrificing two people to save 8 out of 40 passengers. They found that there was a significantly higher level of agreement with the plan to save 8 out of 10 passengers. They argue that the proportional magnitude of the outcome mattered in these judgments.

As previously mentioned, the factory scenario's bad outcomes condition, in which a man suffers a work-related death, also did not differ significantly from the promoral prototype. The bad outcomes condition in both promoral sets depicted a good social context, good intentions, full consent, and bad outcomes combination. Because this condition produced the same effects as the prototype in the factory scenario, this combination may be an instance that most closely resembles the promoral prototype. Interestingly, the bad outcomes condition in the orphanage scenario, in which children were abused, received the second highest "right" rating among that scenario set. Thus, in each scenario set, the participants were less affected by the bad outcomes condition than the other conflicting immoral properties in the nonprototype conditions. One reason for these high ratings might be due to an intention-outcome disconnect in both scenarios. In other words, the actor did not aim for the bad outcomes. As a result, there was a 
perception that the actor was not responsible, since the harm was unintentional. In the factory scenario, many of the participants believed that the factory death was an "accident" and "not Sam's fault." In the orphanage scenario, some participants felt that Beth was not responsible for the bad outcomes and that the post-adoption abuse was "beyond her control.” Previous research (e.g., Lagnado and Channon, 2008) has found that the disconnect between intention and outcomes and the unforseeability of the negative events often leads to more sympathetic judgments, which may explain why these conditions were rated as "right" despite the negative outcomes.

Another noteworthy observation from the promoral scenario sets is how people responded to the "bad" consent conflict conditions, in which there was an action performed against the will of the target in each scenario. For the most part, the participants judged this condition to be more right than wrong even though there was no consent. This may be attributed to the fact that the actors in the scenarios were guided by good intentions and the results were positive; thus, the targets of the actions benefited in some way. For instance, when Sam wants to help a homeless man and finds a way to force the man to take the job, many of the participants rated this situation as right because the quality of the man's life improved as a result Sam's help. One participant said, "Sam did the right thing, he was concerned about the man's well being." Another said, "Yes because without Sam the man wouldn't be as happy and healthy.” In other words, the promoral action, benevolent intentions, and the improved quality of life weighed more than the lack of consent in some people’s judgments.

On the flip side, the participants were less forgiving when the actor's intentions were malevolent or when the action was considered to be taboo within the social context. 
This suggests that bad intentions and bad social context orientations carry a lot of weight in predominantly promoral scenarios. In both promoral scenario sets, the bad intentions variable created the greatest effect sizes when compared to the prototype conditions (orphanage, $d=2.57$; factory, $d=3.08$ ), while the bad social context variable produced the second greatest effect sizes when compared to the prototype condition (orphanage, $d$ $=2.44$; factory, $d=1.43$ ). The strength of these variables is also supported by the qualitative responses. When the participants responded to these conditions, they most often included concerns about the immoral social context property or the immoral intentions of the actor. For example, when responding to the orphanage scenario's bad intentions conflict condition, a number of participants used intentions to justify their judgment ratings and stated that the actor was "self-serving" and wrong because of her ill will. In the factory scenario's bad intentions conflict condition, the participants believed that the action was wrong because Sam's desire was to be mean and cause harm. In the bad social context conflict condition of the factory scenario, some participants felt that lawfully allowing a 5-year-old to work was wrong despite the positive intentions, full consent, and happy ending. In the orphanage scenario, a number of participants condemned the actor's decision to auction babies to generate money for her charity, even though the action was guided by good intentions, consent, and positive outcomes.

Blame/Praise. As predicted, none of the nonprototype conditions were more blameworthy than the immoral prototypes or praiseworthy than the promoral prototypes, and the immoral and promoral prototype conditions significantly differed from most of the nonprototype conditions in their respective sets. The only exception was in the cloning scenario. The immoral cloning prototype condition did not differ from the good 
social context conflict condition or the good intentions condition. As mentioned in the previous section, the combination of the bad intentions, lack of consent, and bad outcomes may have carried enough weight to create a condition that acted similarly to the prototype.

The finding that the good intentions condition received just as much blame as the immoral prototype is surprising. Attribution theorists found that when actions are driven by bad intentions and result in the intended bad outcomes, blame is intensified (Malle \& Bennett, 2002; Ohtsubo, 2007). In contrast, good intentions often mitigate blame (see Weiner, 1995 for review). Under the attribution theory framework, the good intentions condition should have received significantly less blame than the immoral prototype condition. However, the combined effects of the action of cloning, lack of consent, and bad outcomes made the participants less sympathetic, despite the actors' good intentions to help people. One reason for this surprise finding may be that the lack of consent was among the most frequently occurring justifications in this condition and appeared to produce strong effects that minimized the power of the good intentions (see Table XIX to view the comparatively large effect sizes of the consent variable).

Difficulty. Because this framework proposes that the prototypes lack conflict and have properties that are uniformly directed toward the same stance, it was predicted that the prototypes would be easier to judge than the nonprototypes. Surprisingly, there was no consistent pattern among the four scenario sets other than none of the prototypes was more difficult to judge than the nonprototypes, and this variable produced the weakest effect sizes among all three dependent variables $\left(\eta_{\mathrm{p}}{ }^{2} \leq .08\right)$. However, among the immoral scenario sets, the marriage scenario’s immoral prototype and nonprototype 
conflict conditions were statistically similar in difficulty level. The cloning prototype condition was only easier to judge than the good consent and good outcomes conflict conditions, but rated similarly to the social context and good intentions conflict conditions. Among the promoral scenarios, the prototype condition in the orphanage scenario was easier to judge than all nonprototype conflict conditions. However, the factory prototype only differed significantly from the social context conflict condition. These mixed results raise questions about why some single conflict conditions are as easy to judge as moral prototypes, while others are significantly more difficult. For instance, will adding more conflict, such as two conflicting properties (e.g., bad social context and bad intentions against good consent and good outcomes), create a more consistently difficult scenario? Will other research methods lead to the same results? For example, if "difficulty" is measured as the amount of time it takes to cognitively process a judgment, will the response times results be similar to the self-rating results? More information needs to be gathered to understand these inconsistent findings.

\section{Moral Judgment Frequencies and Percentages}

As expected, both the promoral and immoral prototype conditions had near perfect or perfect agreement (i.e., $\geq 99 \%$ ). In the case of the immoral scenario sets, the good social context property also produced a high level of aggregate agreement that the action was wrong. Out of the 111 people who responded, $97-98 \%$ agreed that those conditions were wrong. This suggests that a "good" social context property (e.g., developing a disease-fighting drug or marrying a person you love) did not carry significant weight to affect aggregate agreement when the actions were intentionally malevolent, against the will of others, and resulted in negative outcomes. Even though 
some disagreement became manifest among the nonprototype conflict conditions in the immoral scenario sets, none of the "good" properties carried enough weight to shift the majority of responses towards the "right" end of the rating scale. For instance, the marriage scenario's good intentions conflict condition depicted a structure with a bad social context, good intentions, no consent, and bad outcomes combination. Of the 79 people who responded, $6 \%$ of the participants said that it was the right thing to do while $94 \%$ felt that it was wrong. Thus, even though some of the participants judged the actions in nonprototypes conflict conditions to be right, a greater proportion of the participants rated these conditions as wrong; the single promoral property did not have a big impact in shifting judgments from wrong to right.

Aside from the perfect agreement generated by both promoral prototype conditions, the "bad" outcomes conflict condition (which pitted bad outcomes against good social context, intentions, and consent) also produced a high level of aggregate agreement that the action was right. Out of the 119 people who responded, 94 - 99\% agreed that the bad outcomes condition was right. This suggests that when a socially condoned action (e.g., raising volunteer donations for charity or giving a job to a homeless man) is consensual and driven by benevolent intentions, the disconnected, negative outcomes may not have a strong bearing on our moral judgments. However, in contrast to the fairly consistent judgments of the predominantly immoral nonprototypes discussed earlier above, a single bad property in a predominantly promoral situation had the power to change a high proportion of judgments from "right" to "wrong”. When Sam gave the homeless man a job for malevolent reasons, $62 \%$ of the participants (out of 79 people) rated the action as wrong, despite full consent and the homeless man's improved 
quality of life. Thus, the bad intentions property outweighed the combined promoral properties and resulted in the majority of participants rating that condition as "wrong". Also, the level of disagreement appears to be greater among the conditions in the promoral scenarios sets than in the immoral scenario sets. Among all the conditions across the two promoral scenario sets, the range of agreement that an action was right was between 24 - 100 percent; whereas, among the immoral scenario sets, the range of agreement that the action was wrong was between $82-100 \%$.

These observations support previous research that show how people judge positive and negative actions differently (Malle \& Bennett, 2002; Ohtsubo, 2007; Pizarro et al., 2003). For example, Pizarro and his colleagues (2003) found that purposeful negative behaviors were judged more harshly than impulsive negative behaviors. However, impulsive positive behaviors were rated just as highly as purposeful positive behaviors. Malle and Bennett (2002) found that malevolent intentions intensify blame more than benevolent intentions intensify praise. This asymmetrical effect has also been found in economists' theories of judgment and decision-making. For instance, Kahneman and Tversky (1979) state that, "A salient characteristic of attitudes to changes in welfare is that losses loom larger than gains. The aggravation that one experiences in losing a sum of money appears to be greater than the pleasure associated with gaining the same amount,” p. 279. In other words, a loss of 20 dollars will have a greater impact on decision-making than a gain of 20 dollars. Because there are inferred or actual risks involved in situations that feature immorally oriented properties, people may respond to them as a form of risk aversion. It could be that, under particular conditions, people are more sensitive to the negative or "bad" qualities of a situation (e.g., losses, vices, risk, 
punishment, etc.) than to the positive or "good” qualities (e.g., benevolence, gains, rewards, virtues, etc.) as they make judgments and decisions. The findings in this study support this idea. When a promoral property acted as the conflicting property in a predominantly immoral scenario set (i.e., cloning and marriage), the comparison between the conflict scenario and the prototype produced medium effect sizes when there were significant differences, $d=0.50-0.77$ (in absolute values). Conversely, when an immoral property presented conflict in a predominantly promoral scenario set (i.e., orphanage and factory), the promoral property produced very large effect sizes, $d=0.94$ - 3.08. Thus, these findings show that there was a greater sensitivity to the immoral properties, which supports the asymmetry hypothesis.

\section{Moral Justifications}

In this section, participants were asked to explain the reasons for their moral judgment ratings. The analysis of this qualitative data revealed that certain properties were more salient than others, depending on the scenario and condition. I predicted that the participants would focus on the conflicting property (i.e., the property that is at odds with the others); however, this was not always the case. Even though there was a different conflicting property in each nonprototype condition, the participants tended to rely on a predominant justification when they judged the conditions in the immoral scenario sets. For instance, in the cloning scenario, language about the lack of consent was most often used as a justification across four out of the five conditions (i.e., in all but the full consent conflict condition, which depicts the patients as consenting to the procedure). As one participant stated, "Conducting research on patients without their knowledge or permission was totally unethical.” In the scenario that depicted full 
consent, the participants used intentions (i.e., the malevolent intentions of the medical scientists) as a predominant factor in their justifications. In that condition, a number of participants were concerned that the scientists were in it "for the wealth and power."

Like the cloning scenario, a predominant property also emerged in the marriage scenario. Across all treatment conditions, intention was either used most frequently or was part of a group of statistically similar, most frequently used properties. In the conditions that depicted bad intentions (i.e., Fred marrying for self-serving reasons), some participants felt that Fred was acting out of selfishness and marrying Anne for "all the wrong reasons.” In the good intention conflict condition, some participants felt that Fred's actions were immoral but acknowledged his positive motivations. One person said, "His actions were wrong, even though his intentions were for the best."

A question that emerges as a result of these findings is why the two immoral scenarios produced responses with different predominant properties. Attribution theorists have demonstrated the salience of intentions in judgments of blame (e.g., Lagnado \& Channon, 2008; Malle \& Bennett, 2002; Ohtsubo, 2007). Generally, when the action is deliberate, performed under full awareness, and directly connected to negative outcomes; it is more likely that the actor will receive a higher degree of blame. Using this framework, it is not surprising that intention was often cited as a reason for wrongfulness or blame in the marriage scenario, which depicted the husband's deliberate, self-serving actions as leading to the wife's misery. However, attribution theory does not explain why consent was a salient justification in the cloning scenario’s moral judgments. One explanation could be that some scenarios are more sensitive to issues in the personal domain. As discussed earlier, the personal domain refers to a sphere of actions that are 
not subject to social regulation (see Nucci \& Turiel, 2000 for discussion). It is what the individual considers to be a matter of personal autonomy, preference, and choice. It is possible that this group of participants believes that decisions about one's body is a personal domain issue and subject to consent, as evidenced by this response, "Every patient has the right to know about any test, procedure, or experiment on their body.”

Among the promoral scenarios, the conflicting property stood out more in their respective justifications. For instance, in the "bad" social context conditions, social context concerns were most frequently discussed in justifications in both the factory and orphanage scenarios. Some said, "People should never be sold by anyone. [They] are not possessions," and "I question putting a 5-year-old to work in a factory even if acceptable in the area." Similar results became manifest in the intentions conflict conditions of both scenarios. The participants tended to use language about intentionality, such as, "She shouldn't use people to get ahead, especially children," and "If his true intentions were to make this person miserable, then he is wrong.” In the consent conditions, consent was either part of a pair or a group of the most highly used justifications. Participants made such statements as, “...should allow the community to decide for themselves...” and "Somewhat wrong to force a man into working for him..." In the bad outcomes condition, outcomes and intentions justifications were frequently used in the factory scenario (e.g., “Sam’s actions were right, because he intended to give the needed man a hand," and "He gave this man a job, which enabled him to make a good living.”) However, in the orphanage scenario, intention justifications were used more frequently than outcomes (e.g., "Right because she was devoted to help the orphan children find homes...”). Since many of the participants judged this condition to be "right", the focus 
on the positive intentions was often used to demonstrate the disconnect between the actor's intentions and the actual outcomes in order to absolve him or her or any responsibility for the negative consequences.

Finally, it should also be noted that the promoral prototypes produced a pattern of responses different from the nonprototype conditions. When responding to the promoral prototypes, the participants were more likely to focus on outcomes or provide justifications without moral property language (undefined justifications in particular). For instance, some participants said, “Beth’s actions were right,” and “...turn out good.” Perhaps, with the absence of conflicts in these promoral situations, people tend to focus on the outcomes and/or feel less compelled to justify their judgments, because the judgment may appear to be obvious.

\section{Limitations and Suggestions for Future Research}

There were several limitations to this study. First, not all property combinations were tested. More combinations would have required additional test questions, which would have increased costs. I could have limited the study to two scenario sets (e.g., marriage and factory only) and produced scenarios that covered all of the different combinations. However, since this is exploratory research, I wanted to see if similar patterns emerged from an array of scenarios, which is why four different ones were chosen instead of two. Additionally, I wanted to focus on the single property conflicts to see if particular properties produced greater effects than others. Future research should include multiple property conflicts and test all combinations to see if there are other noteworthy findings. Another limitation to this study is that the population was limited to adults born in America. As a result, the findings in this study cannot be generalized to 
broader populations. Future research should include cross cultural and developmental studies to see there are different effects associated with development and/or culture. Finally, Internet responses tend to be thrifty. Collecting qualitative data via interviews would enhance any new work in this area. Interview opportunities would allow the researcher to capture richer responses and ask follow-up questions.

\section{Conclusion for Study 2}

In sum, the promoral prototype conditions resulted in significantly higher levels of rightness and praise than most or all of the nonprototypes, and the immoral prototypes received higher levels of wrongfulness and blame than most or all of the nonprototypes in each respective scenario set. None of the nonprototype conditions received significantly higher levels of right/wrong or praise/blame than their respective prototype conditions in each scenario set. The difficulty level did not yield consistent patterns across the four scenario sets. The prototypes were easier to judge than all, most, few, or none of the nonprototypes conditions, depending on the scenario set. However, none of the prototypes were found to be more difficult to judge than the nonprototypes. When the participants explained their responses, the type of condition and scenario seemed to have a bearing their justifications. Immoral scenarios produced a predominant justification, while the pormoral scenario justifications were more sensitive to the type of conflict presented in a given condition. Prototypes, across all four scenario sets, resulted in near perfect or perfect agreement. Some nonprototype conflict conditions in the immoral and promoral scenario sets resulted in disagreement, although there was a greater range of disagreement among the nonprototypes in the promoral scenarios. Finally, although no hypothesis testing was conducted to compare promoral and immoral conditions, this 
study appears to contribute further evidence that people judge promoral and immoral situations differently. 


\section{CHAPTER V: GENERAL DISCUSSION AND IMPLICATIONS}

\section{General Discussion}

The question at the heart of this thesis is, "How does moral disagreement become manifest?” I hypothesized that people construct moral prototypes by forming summary, or ideal, representations that have the most characteristics of a moral category. Stimuli that closely resemble immoral prototypes will lead to judgments of wrongfulness and blame while stimuli that closely resemble promoral prototypes will lead to judgments of rightfulness and praise. Within the context of this framework, an immoral prototype is an action that is not condoned by the culture ${ }^{9}$, intentionally self-serving or malevolent, against the will of others, and results in negative consequences. A promoral prototype is an action that is supported by the culture, intentionally benevolent, consensual, and results in positive outcomes. Moral stimuli that deviate from prototypes are nonprototypes. Because nonprototypes do not fit neatly into a category, they can be difficult to judge and act as a source of moral disagreement.

The results of these two studies show that the varied structural representations of the moral properties (i.e., the orientations of the social context, intentions, consent, and outcomes properties) led to repeating patterns of moral judgment. In both studies, all prototypes resulted in perfect or near perfect agreement that the actor was wrong or right. This suggests that moral agreement can be obtained when the social context, intentions, consent, and outcomes properties are uniformly aligned. Additionally, none of the nonprototypes produced judgments that were more wrong or blameworthy than the immoral prototypes or more right and praiseworthy than the promoral prototypes. As a result, prototypes appear to produce judgments that fall at the extreme ends of the right - 
wrong continuum. Most of the nonprototypes produced significantly different judgments than the prototypes and often acted as a source of moral disagreement by producing greater divisions of judgments between right and wrong. There were a few instances in which the nonprototypes were judged similarly to the prototypes, which suggests that the characteristics of those configurations may be within close proximity to the prototype, producing similar effects. For example, good social context, bad intention, no consent, and bad outcomes might be more likely to produce judgments that are similar to immoral prototypes while good social context, good intentions, full consent, and bad outcomes might be more likely to produce judgments that are similar to the promoral prototypes. Surprisingly, the participants did not consistently rate the single conflict nonprototypes as more difficult than the prototypes. However, this finding may be limited to single property conflicts. If the properties are evenly divided (i.e., two properties are oriented towards a promoral stance and two are oriented towards an immoral stance) will these types of nonprototypes lead to situations that are more difficult to judge? Will difficulty level increase linearly as scenarios move from no conflict, single conflict, multiple conflicts? These are questions for future research.

When the participants justified their moral judgments, language about particular properties appeared to be more salient under specific conditions. Previous research on attribution theory led to predictions that the actor's intentions and the outcomes of the situation would have a strong bearing on moral judgments. It has been found that people consider these properties as they determine whether or not to assign blame to actors (e.g., Lagnado \& Channon, 2008; Malle \& Bennett, 2002; Ohtsubo, 2007). Generally, when the action is deliberate, performed under full awareness, and directly connected to the 
negative outcomes; it is more likely that the actor will receive a higher degree of blame, thus, intensifying blame. With such previous findings, it is not surprising that the orientations of these properties, intentions in particular, had a bearing on moral judgment among the participants in both Study 1 and Study 2.

However, the two studies in this research also show that consent issues play an important role in making moral judgments. For example, consent was a dominant property in both the killing (Study 1) and cloning (Study 2) scenario sets. Interestingly, much of the consent research pertains to the body and is found in the areas of bioethics, medical treatments, and the recruitment/participation of research subjects (e.g., Bloch \& Salzberg, 2003; Davies, 2005; Frith, 2005). Perhaps, research on consent is salient in these areas, because these topics often include debates about the degree to which one's body falls within the personal domain. Should parents be allowed to deny their children medical treatment? Should euthanasia be legalized? Should circumcision practices be abandoned? Although the personal domain is culturally and developmentally sensitive, Nucci and Turiel (2000) believe that the personal domain functions as a foundation for identifying rights to personal freedoms and that overstepping these boundaries may promulgate moral concerns about fairness, justice, and welfare. If the body is a strong personal domain issue and the participants believe that decisions about the body should be protected by personal rights to freedoms, then the perceived violation of these rights could be why consent became a dominant justification in the cloning and killing scenarios. In both of these scenarios, the participants often justified their moral judgments by including language about the level of autonomy one should be afforded when making decisions about one's own body. Because consent is an important factor in 
making moral judgments, more research is needed to understand cultural perceptions of consent, additional areas or situations that are particularly sensitive to consent issues, and what full consent entails (i.e., Are there different degrees of consent?).

Finally, in addition to consent, intentions, and outcomes, the social context property also had a significant impact on moral judgments, particularly among the predominantly promoral scenario sets in Study 2. These scenarios demonstrated that a situation could portray good intentions, full consent, and positive outcomes and, yet, still yield judgments of wrongfulness. As described earlier, this model proposes that the social context property can take on a valence of good or bad as a result of the perceiver's conceptual understanding of the action as it is shaped by one's values, beliefs, and informational assumptions. For example, being truthful is considered to be a virtue in our culture and children are often taught that they should tell the truth. However, there may be times when it is appropriate to lie to another person when we consider the context of the lie and intentions of the agent (see Flanagan, 1996 for a philosophical discussion).

In line with this framework, the bad social context property, when pitted against good intentions, full consent, and positive outcomes, produced a nonprototype. This condition led to disagreement about whether a 5-year-old should work or whether babies should be auctioned to raise money for charity; some people rated it as right, while others judged it to be wrong (refer back to Table XVIII to see a breakdown of right and wrong responses). To explain why some people rated these conditions as wrong even when there were good intentions, full consent, and positive outcomes, this model proposes that a conceptual threat influenced some moral judgments. A conceptual threat entails the pervasiveness of a concept's associated harm, risk, or threat, even when the 
circumstances of the situation are promoral. In other words, one reason why some people judged child labor and auctioning babies to be wrong may be due to their inability to disassociate the perceived threat from the concept's constructed meaning no matter how benign the action is rendered.

The scenarios in this research are similar to Haidt's (2001) incest scenario, being that the intentions, consent, and outcomes were manipulated to produce a risk-free situation. Like the findings in Study 2, Haidt observed that a number of people rated incest as wrong, even though the situation was constructed to be harmless. In his scenario, the brother and sister are adults, the intercourse is fully consensual, pregnancy is prevented, and the siblings' relationship is strengthened. He describes his participants as being morally dumbfounded when asked to make a judgment about this scenario, because they were only able to justify their judgments with a "gut feeling". He concluded that the participants relied on moral intuitions to form a judgment.

One explanation for this moral dumbfounding may lie in how the scenario was constructed (see Jacobson, in press for a philosophical critique on Haidt's studies and conclusions). In the incest scenario, the siblings did not suffer any emotional problems and there was no chance of producing children with genetic defects (Haidt, 2001, p. 814). Therefore, anticipated justifications for arguing against incest were taken off the table. By removing these common justifications, the ability to reason about the situation becomes impeded. Even though the risk was stripped from the situation, it is possible that a strong and intact associated threat remained linked to the concept of incest, which may have made it impossible for some people to conceive of it as benign and acceptable. Thus, if one develops an understanding of a situation to carry an inherent risk or harm, 
then the persistence of the conceptual threat may be strong enough to lead to judgments of wrongfulness even when the scenario is framed by promoral attributes. In other words, if the study is designed not to accept common reasons that justify why an action like incest is wrong, then a forced inarticulateness may be the result.

In essence, the two studies in this research support a moral prototype hypothesis. The high percentage of agreement suggests that the prototypes are clear and easy to categorize as either right or wrong. Some researchers believe that as children develop and interact with their environments, they adapt by organizing schemas and different domains of social knowledge; the coordination and use of this knowledge leads to more efficient thought processes (Piaget, 1936/1952; Kolberg, 1969; Turiel, 2010). If prototypes are clear and easy to identify, then it is likely that they also produce rapid responses. Recent cognitive research in social domain theory supports this notion. Lahat, Helwig, and Zelazo (2012) asked research participants to make judgments about rules modeled after social convention and moral prototypes (i.e., actions that clearly fit into the conventional or moral domain without any domain overlap - refer back to literature review for information on social domain theory). The researchers discovered that the neural processing of these domain specific stimuli differed under specific circumstances. One of the findings was that the judgments of conventional prototypes were slower than judgments of moral prototypes when a rule was assumed to prohibit a violation. This observation, along with other cognitive measures performed in the study, suggests that moral prototypes require less effortful processing than social convention prototypes. The authors speculate that moral prototypes require less cognitive effort, because they contain intrinsic negative consequences, while conventional prototypes produce more cognitive 
conflict by requiring the consideration of context and societal rules. Additionally, Richardson, Mulvey, and Killen (see 2012 for review) believe that judgments are influenced by the interplay of two modes of thinking: the experience and the representational systems. The experience system is nonreflective, more automatic, and depends on previous experience, while the representational system is more reflective, effortful, and focused on problem solving. The authors contend that the experience system may kick into effect when common prototypical moral stimuli are encountered. However, the representational system will override it when more complex and/or nonprototypical stimuli are encountered. If this is true, then as children construct categories of what is right and wrong, they probably develop refined neural networks that allow for quick responses to recurring, prototypical moral stimuli in the social space. The further a nonprototypic situation deviates from existing moral prototypes, the more likely judgment will require effortful processing and the engagement of the representational system, providing that cognitive resources are available to attend to the stimulus.

If moral prototypes lead to effortless quick judgments, it is important for researchers not to conclude that a lack of conscious deliberation is evidence that moral judgments are intuitive or "slaves to passion" (see Haidt, 2001 for discussion on intuition and moral judgments). The problem with using a word like "intuitive" to describe moral judgements is that the connotation emphasizes an instinctual, affective nature of moral judgments while minimizing the role of rationality.

If intuition is defined as a cognitive process that is characterized by a lack of conscious deliberation, moral prototypes may act like intuitive responses as a result of their clear representations of particular moral categories and ease of categorization. 
However, it doesn't mean that deliberation never occurred or that the process is completely irrational or non-rational. Reasoning may have occurred at an earlier point in time, such as when a young child first learns to discriminate between the properties that make one category differ from another. When a parent asks her son if he hit his sister "on purpose" or "by accident”, they are reasoning about intentionality and perhaps constructing moral categories of blame. When a teacher asks his students to imagine being a slave working on a plantation, the empathic responses are being used to reason about welfare, justice, and autonomy concerns, which may be assimilated into existing schemas or the catalyst for moral category adaptation. With repeated exposure and practiced use of these categories, it is likely that familiar stimuli will be easier to categorize and result in more rapid responses, thus, eliminating the need for conscious deliberation.

In addition to the findings that moral prototypes are easy to categorize, the results of this research are evidence that moral judgments can be predictable and logical when the properties are harmonious and represent prototypical situations. Actions like the malevolent, volitional, intentional murder of an unwilling victim will not require many people to engage in conscious deliberation, because the moral properties are in-sync and do not present conflict. Alternatively, when nonprototypical stimuli are not easily categorized, then deliberate reasoning may be required. For example, if a wellintentioned, concerned, son places his elderly mother into a nursing home against her will, a conflict is created between the intentionality of the action and the consent/personal autonomy of the parent. If the culture frowns upon placing parents in nursing homes, but the outcomes promote the safety and well-being of the parent, then additional layers of 
complexity are added to the scenario. Such scenarios with multiple conflicts of properties may never be uniformly resolved in a given social milieu, because there is no way to untangle the competing conflicts. When the properties are in conflict, nonprototypical moral stimuli contain elements that are both good and bad and, thus, will probably lead to disagreement.

The findings of this research showed that nonprototypes were more likely to lead to hetergeneous responses, which resulted in some people categorizing the stimuli as right and others categorizing them as wrong; in other words, unlike moral prototypes, the judgments of nonprototypes were less likely to be uniform. As hypothesized earlier, the reason why the nonprototypes produce greater heterogeneity in judgment is that they do not fit neatly into the prototypical moral categories. Some of the more complex nonprototypes may be so difficult to categorize that encountering them would force us to engage in more effortful processing to reach a conclusion and, in some cases, may be too challenging for some people to judge. The difficulty of processing some nonprototypes is depicted in a famous Saturday Night Live skit in which the characters meet an androgynous looking person named Pat. In this skit, the characters observe that Pat posses both traditionally male and female traits, which confuses people. This state of confusion is so uncomfortable that the characters go to great lengths to identify Pat's sex by scheming and crafting specific questions, hoping to obtain clues that will enable them to finally categorize Pat as either a man or woman.

OFFICE WORKER: Do you have a girl.. uh, a boy... uh... Are you married?

PAT: I was going to be married, but then it all fell apart.

OFFICE WORKER: Aw. What happened? 
PAT: I don’t know. Ask my ex-fiancé.

OFFICE WORKER: [Excited, because he thinks he will finally get a clue.] What's your ex-fiancé's name?

PAT: Terry

The characters in this skit are never successful at categorizing Pat, which forces them to engage in more effortful processing as they interact with Pat (i.e., by crafting specific, information-seeking questions). A similar experience may be true of moral nonprototypes. The more complicated the moral stimulus is to classify, the more one will grapple before drawing a conclusion. Some people may employ logistics, draw from experiences, participate in empathic reasoning (e.g., imaging what it would be like to be in someone else's shoes), conduct a cost-benefit analysis, or engage in deep reflection (such as defining what is morally good or bad) before making a judgment.

Because some complex nonprototypes are difficult to categorize as right or wrong, McIntyre (1981) and Posner (1999) are probably correct - that some moral issues cannot be resolved and are incommensurable. These types of situations may not be amenable to an agreed upon moral resolution and will always result in moral disagreement as long as conflict persists. Abortion is likely to be one of those issues, because it pits the welfare of the fetus against the welfare and autonomy of the mother. For example, in 2006, Nicaragua enforced very strict anti-abortion laws. In 2011, a 12year-old Nicaraguan girl was raped and impregnated by her stepfather and not allowed to have an abortion. When the pregnancy began to endanger her life, she was forced to give birth by cesarean section at 36 weeks (12-year-old Rape Victim Gives Birth, 2011). This case was extremely controversial and led to an uproar by activists on both sides of the 
issue. Some Nicaraguans challenged the government to consider revising or reversing the law so that abortions can be used to protect the lives of the mothers. However, religious figures and politicians held fast to their cause of protecting the lives of fetuses (Downs, 2011). Even in a culture that is predominantly Catholic (a religion that supports a pro-life viewpoint), Nicaraguans could not reach a unanimous conclusion about abortion. Because protecting the life of the mother vs. fetus debate is a highly charged conflict, obtaining social resolution in which everyone agrees and forms the same judgment may be impossible.

Even though complex moral nonprototypes are likely to result in unresolved disagreement, I agree with MacIntyre’s (1981) assertion that morality is not simply emotivism and not based on individual preferences, attitudes, and feelings. If moral representations were purely intuitive and largely driven by emotions or "gut feelings", then the responses to prototypical stimuli would probably vary according to individual preferences, experiences, and emotional states. However, the high percentage of agreement to prototypical situations in these two studies show that some moral stimuli are easy to categorize and reliably produce predictable moral judgments among the diverse adults who participated in this research.

The fact that moral agreement can be obtained under specific conditions suggests that there may be some moral "truths". However, as Nussbaum (1993) suggests, the moral "truth" may lie in the substrate of morality and not necessarily in its content. Even though the content of moral situations may evolve over time, the relationship between the properties may be a stable, fundamental aspect of moral category construction. Churchland (1998) suggests that as society evolves, we will be confronted with new 
technologies, information, and experiences. For example, one current problem we are experiencing in this nascent digital age is how our personal information will be protected against the clandestine software that tracks our Internet activity. Many of the new technologies provide a means for others to track our physical locations, purchases, and web browsing activity; identify people from photos using face recognition software; and allow hackers to steal our identities. In our current social context, gathering information by Internet is a routine social practice. However, when companies are asked to express why they are loading tracking software onto peoples’ computers, concerns about intentions and intentionality are being addressed. Are they using this information for the public good or do power and money primarily motivate them? Additionally, what makes these tracking capabilities particularly frightening is that much of this activity is conducted without our knowledge, which is a consent issue that wasn't present 50 years ago (see What They Know, 2012). Finally, we evaluate the outcomes of such practices, such as criminals gaining access to our personal information vs. the creation of more personalized Internet experiences, as we try to draw conclusions about this technology in the moral domain. In essence, the evolution of society may lead to new moral content concerns; however, we will continue to use the same properties (i.e., social context, intentions, consent, and outcomes properties) to make sense of these situations as we form moral judgments.

In the context of this prototype framework, moral truths may be in how these properties collectively influence our judgments. When the properties are aligned and directed toward the same stance, then moral agreement will become manifest. The further the stimuli deviate from the prototype, the greater the likelihood for moral 
disagreement. In other words, the "truth" may lie in the descriptive account of moral judgments (e.g., describing the relationship between moral properties and moral judgments) and not in normative or prescriptive observations (i.e., accounts of what is perceived to be morally right or wrong within a particular culture).

\section{Implications}

Revision of the Blame Intensification Hypothesis. As previously mentioned, attribution theorists (e.g., Lagnado \& Channon, 2008; Malle \& Bennett, 2002; Ohtsubo, 2007) have found that blame intensifies when there is a direct connection between the actor's intentions and the resulting outcomes. However, the findings in this research suggest that the hypothesis should be revised to include consent and social context properties. The blame intensification hypothesis predicts that the bad intentions leading to the bad outcomes will intensify blame. However, the immoral prototype conditions in both the cloning and marriage scenario sets of Study 2 received significantly higher degrees of blame and wrongfulness than the nonprototype conditions that included the bad intentions and bad outcomes combinations. For example, the bad intentions, bad outcomes, good consent, and bad social context condition was rated as less wrong than the immoral prototype condition. This demonstrates that full consent had a mitigating effect on the bad intentions + bad outcomes combination and that this combination does not always result in the most intensified judgment of wrongfulness and blame. Because the ratings of prototypes consistently resulted in extreme moral judgment, I believe that the intensification hypothesis needs to be revised to include bad social context + bad intentions + bad consent + bad outcomes $=$ intensified blame. Additionally, a promoral condition should be added to the hypothesis as this research shows that good social 
context + good intentions + good consent + good outcomes resulted in the highest levels of rightfulness and praise.

A Moral Judgment Calculus. Another implication of this research is that finding consistent moral judgment patterns could lead to applications that involve the mathematical modeling of moral judgment. In the same way economic models are used to forecast trends or decision models are used to predict individual or group choices, I believe that it is possible to create a moral judgment model that could be used to estimate the predicted level of agreement or disagreement of a moral situation. For example, such a model might predict that approximately 60 - 70\% of population X would consider situation Y to be wrong. Because moral judgment can be described as the degree of "rightness” or "wrongness" of a situation, it can be represented as progression of numerical values on a scale. In these studies, none of the nonprototypes were rated as more wrong or right than the moral prototypes. Therefore, if moral prototypes consistently receive the greatest degrees of “wrongness” or "rightness”, they would mark the two ends of a continuum with a predictive value of near perfect or perfect agreement that the action is either wrong or right. If nonprototypes are more prone to moral disagreement, then their values would likely fall in-between the polar opposite values of the prototypes. The asymmetrical nature of promoral and immoral judgments could also be quantified as a result of the immoral properties carrying more weight and having a greater psychological impact on judgment than promoral properties. Finally, if the accumulation of properties carries weight and has additive effects, then the heightened power of such configurations could also be represented in a mathematical model. If 
future research leads to such a model, the applications would enable people to predict public sentiment about controversial issues, which could inform policymaking.

Connections to Moral Development and Education. The findings in this research demonstrate that making moral judgments involves the coordination of moral properties, which may require more cognitive effort when the situations are nonprototypical. This supports the emerging work on the relationship between moral development and neuroscience. For instance, Lehat et al. (2012) found differences between prototypical moral and conventional transgressions, which demonstrates that not all social judgments require the same degree of executive functioning. Based on a review of literature, Richardson et al. (2012) hypothesize that nonprotypical moral judgments may lead to more effortful processing when coordinating certain variables, such as the source of distress, the characteristics of the victim, perceived illegitimate requests for exclusion, etc. Additionally, the authors speculate that when the situations are embedded in multifaceted contexts that require the coordination of overlapping moral, social, and/or personal domains at once, judgments may not be automatic and may require greater cognitive resources.

Because it was discovered that there are certain age effects associated with executive functioning and particular forms of social judgment (Lehat et al., 2012), further neurophysiological research could enhance or, perhaps, challenge previous conceptions of moral development. Thus, research on children's coordination of moral properties should be conducted to determine whether or not there are developmental effects when responding to nonprototypical vs. prototypical moral stimuli. By understanding the interplay of moral development and neuroprocessing, educators can create curriculum 
approaches that scaffold children as they transition towards social reasoning that involves greater cognitive complexity. These programs could be implemented at varying levels of education or experience and may lead to prosocial interactions, institutional policies, or reforms. For example, understanding the relationship between consent and promoral judgment may lead more people to favor full disclosure of how personal information is used, which could bolster business/client relationships.

Recommendation for Policymakers. Because the overall findings in this study suggest that the language used to describe a moral situation affects judgments, it is important to consider how situations are presented when engaging in moral discourse. In the abstract or in prototypical situations, people might be more likely to use principles, authority/norms or general undefined responses in their justifications. However, as moral situations become more contextualized and deviate from the prototype, people may increasingly focus on the properties or particulars of the situation by weighing the moral properties, identifying a salient property, and/or reflecting on whether or not there were alternative, more desirable solutions available to the actor.

Because moral judgments appear to be sensitive to how the issue is presented, debates about abstract and prototypically moral situations may drive a different type of judgment than those generated from nonprototypical, contexualized experiences. For example, people may feel more ambivalent about a nonprototypical situation, because they are able to see both pros and cons. When these issues are being discussed in the abstract or with limited information, it is also possible that people may be influenced by inferences based on their prototypical conceptual understandings of the issues. This poses problems for policymakers, because the laws, practices, and/or procedures created 
from abstract or prototypical understandings of an issue may not adequately account for the different type of judgments generated from nonprototypical situations.

This was found to be true among school administrators and state lawmakers as they debated the efficacy of zero tolerance policies adopted by many school districts in the 1990's. Zero tolerance practices aimed to punish students who brought weapons or drugs to school without considering varying circumstances. As a result, children were being suspended or punished for bringing any "forbidden” items to school, including plastic butter knives, cold medicine, and inhalers (Associated Press, 2007). The main problem with the policy was in its rigidity. The policy failed to take into account the intentions of the children and did not adequately distinguish what constituted a viable threat within the social context of public schools. Because a number of zero tolerance practices were not flexible enough to account for nonprototypical circumstances, some schools were subject to harsh criticism and even lawsuits (Cauchon, 1999). As a result, many school districts have since abandoned this practice. This is why it is prudent to recognize that while abstract and prototypical moral transgressions often appear to be black or white; contextualized, nonprototypical situations can produce varying shades of gray. Understanding these nuances will help policymakers draft stronger, more flexible practices that can account for varied situations. 


\section{REFERENCES}

12-year-old rape victim gives birth. (2011, November 1). Nicaraguan Dispatch.

Retrieved from http://www.nicaraguadispatch.com/breaking-news/12-year-oldrape-victim-gives-birth/1077

Ames, D. R., Knowles, E. D., Morris, M. W., Kalish, C. K., Rosati, A., D., \& Gopnik, A. (2001). The social folk theorist: Insights from social and cultural psychology on the contents and contexts of folk theorizing. In B. F. Malle, L. J. Moses \& D. A. Baldwin (Eds.), Intentions and intentionality : Foundations of social cognition (pp. xiv, 417 p.). Cambridge, Mass.: MIT Press.

Aristotle. (1999). Nicomachean ethics (T. Irwin, Trans. 2nd ed.). Indianapolis, Ind.: Hackett Publishing Company, Inc.

Associated Press. (2007, June 15). Has 'zero tolerance' in schools gone too far? Some states' lawmakers move in that direction on violence, drugs policies. Retrieved from http://www.msnbc.msn.com/id/19249868/ns/us_news-education/t/has-zerotolerance-schools-gone-too-far/

Assor, A., Roth, G., \& Deci, E. L. (2004). The emotional costs of parents' regard: A self-determination analysis. Journal of Personality, 72(1), 47-88.

Baird, J. A., \& Baldwin, D. A. (2001). Making sense of human behavior: Action parsing and intentional inference. In B. F. Malle, L. J. Moses \& D. A. Baldwin (Eds.), Intentions and intentionality : Foundations of social cognition (pp. 194-206). Cambridge, Mass.: MIT Press.

Barber, B. K. (1996). Parental psychological control: Revisiting a neglected construct. Child Development, 67(6), 3296-3319. 
Bersoff, D. M., \& Miller, J. G. (1993). Culture, context, and the development of moral accountability judgments. Developmental Psychology, 29(4), 664-676.

Bloch, S., \& Salzberg, M. (2003). Informed consent in psychiatric research. Current Opinion in Psychiatry, 16(6), 679-684.

Blasi, A. (1999). Emotions and moral motivation. Journal for the Theory of Social Behavior, 29(1), 1-19.

Brehl, B. A. (2008). Maybe you didn't mean to hurt me, but did you have a good reason? Children's and adolescents' reasoning about the harmful side-effects of nonaccidental behavior (Doctoral dissertation). Dissertation Abstracts International: Section B: The Sciences and Engineering, 69(5-B), (No. 200899220-034).

Bruch, H. (1982). Psychotherapy in anorexia nervosa. International Journal of Eating Disorders, 1(4), 3-15.

Cauchon, D. (1999, April 13). Zero-tolerance policies lack flexibility. USA Today. Retrieved from http://www.usatoday.com/educate/ednews3.htm

Chiu, C.-Y., \& Hong, Y.-Y. (1992). The effects of intentionality and validation on individual and collective responsibility attribution among Hong Kong Chinese. The Journal of Psychology, 126(3), 291-300.

Churchland, P. M. (1996). The neural representation of the social world. In L. May, M. Friedman \& A. Clark (Eds.), Mind and morals : Essays on cognitive science and ethics (pp. 91-108). Cambridge, Mass.: MIT Press.

Churchland, P. M. (1998). Toward a cognitive neurobiology of the moral virtues. Topoi, 17(2), 83-96. 
Cohen, J. (1988). Statistical power analysis for the behavioral sciences (2nd ed.). Hillsdale, N.J.: L. Erlbaum Associates.

Davies, B. R. (2005). Coercion or collaboration? Nurses doing research with people who have severe mental health problems. Journal of Psychiatric and Mental Nursing, 12(1), 106-111.

Dijksterhuis, A., \& Smith, P. K. (2005). What do we do unconsciously? And how? Journal of Consumer Psychology, 15(3), 225-229.

Downs, R. (2011, November 03). Abortion ban in Nicaragua needlessly endangering women's lives, say activists. The Christian Post. Retrieved from http://global.christianpost.com/news/abortion-ban-in-nicaragua-needlesslyendangering-womens-lives-say-activists-60425/

Flanagan, O. (1996). The moral network. In R. N. McCauley (Ed.), The Churchlands and their critics (pp. 192-215). Cambridge, MA: Blackwell Publishers.

Frith, L. (2005). HIV testing and informed consent. Journal of Medical Ethics, 31(12), 699-700.

Geertz, C. (1973). The impact of the concept of culture of Man. In The interpretations of culture. New York: Basic Books, Inc.

Gewirth, A. (1986). Why rights are indispensable. Mind, 95(379), 329-344.

Goodnough, A., Carey, B., Jordan Sexton, C., \& Yardley, W. (2005, April 1). Schiavo dies, ending bitter case over feeding tube. The New York Times, p. 1.

Gowans, C. W. (2000). Introduction. In C. W. Gowans (Ed.), Moral disagreements : classic and contemporary readings (pp. xii, 265 p.). London ; New York: Routledge. 
Greene, J., \& Haidt, J. (2002). How (and where) does moral judgment work? Trends in Cognitive Sciences, 6(12), 517-523.

Haidt, J. (2001). The emotional dog and its rational tail: A social intuitionist approach to moral judgment. Psychological Review, 108(4), 814-834.

Haidt, J. (2003). The emotional dog does learn new tricks: A reply to Pizarro and Bloom (2003). Psychological Review, 110(1), 197-198.

Haidt, J., Koller, S. H., \& Dias, M. G. (1993). Affect, culture, and morality, or is it wrong to eat your dog? Journal of Personality and Social Psychology, 65(4), 613-628.

Hasebe, Y., Nucci, L., \& Nucci, M. S. (2004). Parental control of the personal domain and adolescent symptoms of psychopathology: A cross-national study in the United States and Japan. Child Development, 75(3), 815-828.

Helwig, C. C., Arnold, M. L., Tan, D., \& Dwight, B. (2003). Chinese adolescents' reasoning about democratic and authority-based decision-making in peer, family, and school contexts. Child Development, 74(3), 783-800.

Hermand, D., Mullet, E., Tomera, P., \& Touzart, V. (2001). The relationship between intent, consequences, the dangerousness of the victim, and blame: The case of self-defense. Psychology, Crime, and Law, 7(1), 57-69.

Jacobson, D. (in press). Moral dumbfounding and moral stupefaction. In M. Timmons (Ed.), Oxford studies in normative ethics, volume 2. New York: Oxford University Press.

Jones, E. F., \& Thomson, N. R. (2001). Action perception and outcome valence: Effects on children's inferences and intentionality and moral and liking judgments. The Journal of Genetic Psychology, 162(2), 154-166. 
Kahneman, D., \& Tversky, A. (1979). Prospect theory: An analysis of decision under risk. Econometrica, 47(2), 263-292.

Kampert, P., \& Martinez, M. (2003, October 22). Florida rushes law to place woman back on life support Gov. Bush orders nutrients resumed for comatose Terri Schiavo. Chicago Tribune, p. 1.

Kant, I. (1998). Groundwork of the metaphysics of morals (M. J. Gregor, Trans.). Cambridge, U.K. ; New York: Cambridge University Press. (Original work published in 1785)

Karniol, R. (2003). Egocentricism versus protocentrism: The status of self in social prediction. Psychological Review, 110(3), 564-580.

Kohlberg, L. (1969). Stage and sequence: The cognitive-developmental approach to socialization. In D. A. Goslin (Ed.), Handbook of Socialization Theory and Research (pp. 347-480). Chicago: Rand McNally.

Kohlberg, L., Levine, C., \& Hewer, A. (1983). Moral stages : a current formulation and a response to critics. New York: Karger.

Korsgaard, C. M. (1998). Introduction (M. J. Gregor, Trans.). In I. Kant (Ed.), Groundwork of the Metaphysics of Morals (pp. vii-xxx). Cambridge, U.K.: Cambridge University Press.

Krebs, D. L., \& Denton, K. (1997). The forms and functions of real-life decision-making. Journal of Moral Education, 26(2), 131-145. 
Krebs, D. L., Denton, K., Wark, G., Couch, R., Racine, T., \& Krebs, D. L. (2002). Interpersonal moral conflicts between couples: effects of type of dilemma, role, and partner's judgments on level of moral reasoning and probability of resolution. Journal of Adult Development, 9(4), 307-316.

Lagnado, D. A., \& Channon, S. (2008). Judgments of cause and blame: The effects of intentionality and foreseeability. Cognition, 108(2), 754-770.

Lahat, A., Helwig, C. C., \& David, P. (2012). Age-related changes in cognitive processing of moral and social conventional violations. Cognitive Development, 27(2), 181-194.

Lakoff, G. (2002). Moral politics: How liberals and conservatives think. Chicago: University of Chicago Press.

Leonard, L. (2001). Adopting female "circumcision" in southern Chad: The experience of Myabe. In B. Duncan-Shell \& Y. Hernlund (Eds.), Female 'circumcision' in Africa: Culture, controversy, and change. (pp. 168-192). Boulder, CO: Lynn Rienner.

Linacre, J. M. (2004). Optimizing rating scale category effectiveness. In E. V. Smith \& R. M. Smith (Eds.), Introduction to rasch measurement (pp. 258-278). Maple Grove, MN: JAM Press.

MacIntyre, A. C. (1981). After virtue: A study in moral theory. Notre Dame, IN: University of Notre Dame Press.

Malle, B. F., Moses, L. J., \& Baldwin, D. A. (2001). Introduction. In B. F. Malle, L. J. Moses \& D. A. Baldwin (Eds.), Intentions and intentionality: Foundations of social cognition (pp. 1-24). Cambridge, Mass.: MIT Press. 
Markman, A. B., \& Gentner, D. (2001). Thinking. Annual Review of Psychology, 52, 223-247.

McKenna, J. J. (2002, September-October). Breastfeeding and bedsharing still useful (and important) after all these years. Mothering Magazine, 114, 7-8.

McNelis, J. (2003, October 27). End suffering. Chicago Tribune, p. 20.

Medin, D. L. (1989). Concepts and conceptual structure. American Psychologist, 44(12), $1469-1481$.

Medin, D. L., \& Smith, E. E. (1984). Concepts and concept formation. Annual Review of Psychology, 34, 113-138.

Mele, A. R. (2001). Acting intentionally: Probing folk notions. In B. F. Malle, L. J. Moses \& D. A. Baldwin (Eds.), Intentions and intentionality: Foundations of social cognition (pp. 27-43). Cambridge, Mass.: MIT Press.

Malle, B. F., \& Bennett, R. E. (2002). People's praise and blame for intentions and actions: Implications of the folk concept of intentionality. Technical Reports of the Institute of Cognitive and Decision Sciences, (No. 02-2).

Moses, L. J. (2001). Some thoughts on ascribing complex intentional concepts to young children. In B. F. Malle, L. J. Moses \& D. A. Baldwin (Eds.), Intentions and intentionality: Foundations of social cognition (pp. 69-83). Cambridge, Mass.: MIT Press.

Nelson-Le Gall, S. (1984). Children's and adults assignment of blame for personal injury. The Journal of Psychology, 117, 135-142. 
Nelson-Le Gall, S. (1985). Motive-outcome matching and outcome foreseeability: Effects on attribution of intentionality and moral judgments. Developmental Psychology, 21(2), 332-337.

Nucci, L. (1985). Children's conceptions of morality, societal convention, and religious prescription. In C. Harding, ed., Moral dilemmas: Philosophical and psychological issues in the development of moral reasoning (pp. 115 - 136). Chicago: Precedent Press.

Nucci, L. (2001). Education in the moral domain. Cambridge: Cambridge University Press.

Nucci, L., Camino, C., \& Sapiro, C. M. (1996). Social class effects on northeastern Brazilian children's conceptions of areas of personal choice and social regulation. Child Development, 67(3), 1223-1242.

Nucci, L., \& Smetana, J. G. (1996). Mothers' concept of young children's areas of personal freedom. Child Development, 67(4), 1870-1886.

Nucci, L., \& Turiel, E. (1993). God's word, religious rules, and their relation to Christian and Jewish children's concepts of morality. Child Development, 64(5), 14751491.

Nucci, L., \& Turiel, E. (2000). The moral and personal: Sources of conflicts. In L. Nucci, G. Saxe, \& E. Turiel (Eds.) Culture, thought, and development (pp. 115137). MahWah, NJ: Erlbaum.

Nucci, L., \& Weber, E. K. (1995). Social interactions in the home and the development of young children's conceptions of the personal. Child Development, 66(5), 14381452. 
Nussbaum, M. C. (1993). Non-relative virtues: An Aristotelian approach. In M. C. Nussbaum \& A. K. Sen (Eds.), The quality of life (pp. 242-270). Oxford ; New York: Clarendon Press.

Nussbaum, M. C. (1999). Judging other cultures: The case of genital mutilation. In Sex and Social Justice (pp. 118-129). New York: Oxford University Press.

Ohtsubo, Y. (2007). Perceived intentionality intensifies blameworthiness of negative behaviors: Blame-praise asymmetry in intensification effect. Japanese Psychological Research, 49(2), 100 - 110.

Okin, S. M. (1999). Is multiculturalism bad for women? In S. M. Okin (Ed.), Is multiculturalism bad for women? (pp. 9-24). Princeton, NJ: Princeton University Press.

Olthof, T., Ferguson, T. J., \& Luiten, A. (1989). Personal responsibility antecedents of anger and blame reactions in children. Child Development, 60, 1328-1336.

Pashler, H., Johnston, J. C., \& Ruthruff, E. (2001). Attention and performance. Annual Review of Psychology, 52, 629-651.

Piaget, J. (1952). The origins of intelligence in children. (M. Cook, Trans.). New York: International Universities Press. (Original work published in 1936)

Piaget, J. (1965). The moral judgment of the child (M. Gabain, Trans. 1st Free Press paperback ed.). New York: Free Press. (Original work published in 1932)

Pinker, S. (2002). The blank slate: The modern denial of human nature. New York: Viking.

Pizzaro, D. A., \& Bloom, P. (2003). The intelligence of moral intuitions: Comment on Haidt (2001). Psychological Review, 110(1), 193-196. 
Pizzaro, D. A., Uhlmann, E., \& Salovey, P. (2003). Assymetry in judgments of moral blame and praise: The role of perceived metadesires. Psychological Science, 14, 267-272.

Posada, R., \& Wainryb, C. (2008). Moral development in a violent society: Colombian children's judgments in the context of survival and revenge. Child Development, 79(4), 882-898.

Posner, R. A. (1999). The problematics of moral and legal theory. Cambridge, Mass.: Belknap Press of Harvard University Press.

Povinelli, D. J. (2001). On the possibilities of detecting intentions prior to understanding them. In B. F. Malle, L. J. Moses \& D. A. Baldwin (Eds.), Intentions and intentionality : Foundations of social cognition (pp. 225-248). Cambridge, Mass.: MIT Press.

Rai, T. S., \& Holyoak, K. J. (2010). Moral Principles or Consumer Preferences? Alternative Framings of the Trolley Problem. Cognitive Science, 34(2), 311-321.

Reeder, G. D., Vonk, R., Ronk, M. J., Ham, J., \& Lawrence, M. (2004). Dispositional attribution: Multiple inferences about motive related traits. Journal of Personality and Social Psychology, 86(4), 530-544.

Richardson, C. B., Mulvey, K. L., \& Killen, M. (2012). Extending social domain theory with a process-based account of moral judgments. Human Development, 55(1), 425.

Ryan, R. M., \& Deci, E. L. (2000). The "what" and "why" of goal pursuits: Human needs and the self-determination of behavior. Psychological Inquiry, 11, 227-268. 
Shweder, R., Balle-Jensen, L., \& Goldstein, W. (2003). Who sleeps by whom revisited. In R. Shweder (Ed.), Why Do Men Barbeque? Recipes for Cultural Psychology (pp. 46-73). Cambridge, MA: Harvard University Press.

Shweder, R. A. (1984). Anthropology's romantic rebellion against the enlightenment, or there is more to thinking than reason and evidence. In R. A. Shweder \& R. A. LeVine (Eds.), Culture Theory (pp. 27-66). Cambridge: Cambridge University Press.

Shweder, R. A. (2003a). Introduction: Anti-postculturalism (or the view from manywheres). In R. A. Shweder (Ed.), Why do men barbecue?: Recipes for cultural psychology (pp. 1-45). Cambridge: Harvard University Press.

Shweder, R. A. (2003b). What about female genital mutilation? And why understanding culture matters. In R. A. Shweder (Ed.), Why do men barbecue: Recipes for cultural psychology (pp. 168-216). Cambridge, MA: Harvard University Press.

Shweder, R. A., Mahapatra, M., \& Miller, J. G. (1987). Culture and moral development. In J. Kagan \& S. Lamb (Eds.), The emergence of morality in young children (pp. 1-82). Chicago: University of Chicago Press.

Shweder, R. A., Much, N. C., Mahapatra, M., \& Park, L. (2003). The "big three" of morality (autonomy, community, divinity) and the "big three" explanations of suffering. In R. A. Shweder (Ed.), Why do men barbecue? Recipes for cultural psychology (pp. 74-133). Cambridge, MA: Harvard University Press.

Smetana, J. G. (1981). Adolescent and young adult women's reasoning about abortion. Journal of Applied Developmental Psychology, 2(3), 211-226. 
Smetana, J. G. (1983). Social-cognitive development: Domain distinctions and coordinations. Developmental Review, 3(2), 131-147.

Smetana, J. G. (2005). Adolescent-parent conflict: Resistance and subversion. In L. Nucci (Ed.), Conflict, contradiction, and contrarian elements in moral development and education (pp. 69-92). Mahwah, New Jersey: Lawrence Erlbaum Associates.

Stone, M. H. (2004). Substantive scale construction. In E. V. Smith \& R. M. Smith (Eds.), Introduction to rasch measurement (pp. 201-225). Maple Grove, MN: JAM Press.

Terri Schiavo and the moral divide. (2005, March 29). The New York Times, p. 16.

Tabachnick, B. G., \& Fidell, L. S. (2001). Using multivariate statistics. Boston: Allyn and Bacon.

Turiel, E. (2002). The culture of morality : social development and social opposition. New York: Cambridge University Press.

Turiel, E. (2010). Snap judgment? Not so fast: Thought, reasoning, and choice as psychological realities. Human Development, 53(3), 105-109.

Turiel, E., Hildebrandt, C., \& Wainryb, C. (1991). Judging social issues: Difficulties, inconsistencies, and consistencies. Monographs of the Society for Research in Child Development, 56(2), (Serial No. 224).

Waal, F. B. M. d. (1996). Good natured : The origins of right and wrong in humans and other animals. Cambridge, Mass.: Harvard University Press.

Wainryb, C. (1993a). The application of moral judgments to other cultures: Relativism and universality. Child Development, 64(3), 923-933. 
Wainryb, C. (1993b). Understanding differences in moral judgments: The role of informational assumptions. Child Development, 62(4), 840-851.

Wainryb, C., \& Ford, S. (1998). Young children's evaluations of acts based on beliefs different from their own. Merrill-Palmer Quarterly, 44(4), 484-503.

Weiner, B. (1985). An attributional theory of achievement motivation and emotion. Psychological Review, 92(4), 548-573.

Weiner, B. (2001). Repsonsibility for social transgressions: An attributional analysis. In B. F. Malle, L. J. Moses \& D. A. Baldwin (Eds.), Intentions and Intentionality: Foundations of social cognition (pp. 331-344). Cambridge, MA: The MIT Press. Wellman, H. M., \& Phillips, A. T. (2001). Developing intentional understandings. In B. F. Malle, L. J. Moses \& D. A. Baldwin (Eds.), Intentions and intentionality : Foundations of social cognition (pp. 125-148). Cambridge, Mass.: MIT Press.

What they know [Special Series] (2012). The Wall Street Journal. Retrieved from http://online.wsj.com/public/page/what-they-know-digital privacy.html?mod=WSJ_topnav_na_tech]

Willinger, M., Ko, C.-W., Hoffman, H., Kessler, R. C., \& Corwin, M. J. (2003). Trends in infant bed sharing in the United States 1993 - 2000: The national infant sleep position study. Archives of Pediatrics and Adolescent Medicine, 157(1), 43-49.

Wilson, J. Q. (1993). The moral sense. New York: Free Press.

Yau, J., \& Smetana, J. G. (2003a). Adolescent-parent conflict in Hong Kong and Shenzhen: A comparison of youth in two cultural contexts. International Journal of Behavioral Development, 27(3), 201-211. 
Yau, J., \& Smetana, J. G. (2003b). Conceptions of moral, social-conventional, and personal events among Chinese preschoolers in Hong Kong. Child Development, 74(3), 647-658.

Zelazo, P. D., Helwig, C. C., \& Lau, A. (1996). Intention, act, and outcome in behavioral prediction and moral judgment. Child Development, 67, 2478-2492. 


\section{ENDNOTES}

${ }^{1}$ In philosophy, teleological is derived from the Greek word telos, which means ultimate end. Teleological beliefs are beliefs that harmonize with what we perceive to be a natural purpose in life.

${ }^{2}$ Post-conventional moral reasoning is characterized by approaching moral dilemmas from a frame of justice and objective moral standards from which moral truths can be identified. According to Kohlberg, this is the highest stage of moral development.

${ }^{3}$ The study classified the subjects according to the following racial groups: White, Hispanic, African-American, and Asian/Other.

${ }^{4}$ We need to identify different forms of consent, which appear to range from fully autonomous to less than self-determined. For example, when a patient signs a consent form to free a doctor from future liability in order to be treated, is the patient fully consenting to the terms or does the threat of not being treated pressure him to consent? Is the choice to become a prostitute a fully autonomous decision, or are there external pressures that force one to make such a decision?

${ }^{5}$ Not everyone in our society is in a position to act autonomously. Children and the mentally challenged often require the consent of a guardian before engaging in particular actions. Thus, age and ability to reason might be two variables that determine who has the right to consent within particular domains.

${ }^{6}$ Since intentionality and outcomes are often presented together in the empirical research, both will be discussed in the same section.

${ }^{7}$ According to Weiner's attributional theory $(1995,2001)$, there are three underlying properties to causality: (a) locus (i.e., whether the cause is within or outside of 
the agent), (b) stability (i.e., whether or not the cause is constant or temporary), and (c) controllability (some causes are subject to volition whereas others are beyond the agent's will).

${ }^{8}$ The informational assumptions and values constructed within a particular context influence how a moral situation is interpreted. (Refer to Part II for a more detailed explanation of social context.)

${ }^{9}$ In this case, morality is not being reduced to cultural norms or agreement. Instead, this framework asserts that cultures generally construct norms in line with the attributes that distinguish promoral prototypes from immoral prototypes. Thus, culture provides a context for how the action is interpreted and understood within a particular context, and this cultural interpretation of an action can assume a valence of "good" or "bad". 


\section{APPENDIX}

Surveys for Studies 1 and 2

\section{STUDY 1}

\section{SURVEY 1 - ABSTRACT PROTOTYPES}

DIRECTIONS: This questionnaire will ask you to read four different statements and to respond to a set of corresponding questions. It is very important to read each statement and to respond to each question. Your input is greatly appreciated. Thank you for your participation!

1. The following prompts were given:

a. It is to kill another person.

b. It is to steal from another person.

c. It is to destroy another person's property.

d. It is to forcefully punch another person in the back.

The participants used the following scale to fill in the blanks.

very wrong

wrong

somewhat more wrong than right

somewhat more right than wrong

right

very right

2. After each stimulus, the participants were asked to please explain why and write a qualitative response.

(SURVEYS 2 - 5)

DIRECTIONS: This questionnaire will ask you to read four different scenarios and to respond to a set of corresponding questions. It is very important to read each scenario carefully and to respond to each question. Your input is greatly appreciated. Thank you for your participation!

\section{QUESTIONS ASKED:}

[After each scenario, the participants were asked the same questions, which are as follows:]

1. Complete the following sentence by selecting a response listed below:

It was for (name) to (action - e.g. steal).

very wrong

wrong 
somewhat more wrong than right

somewhat more right than wrong

right

very right

2. Please explain why you selected that response.

\section{SURVEY 2 - INTENTIONALITY HYPOTHETICALS}

SCENARIO ONE: Dr. Smith is a medical doctor. Her patient, Sarah, was dying of cancer. Although Sarah was able to think clearly, she was in a constant state of pain and suffering. Dr. Smith cared about Sarah and wanted to relieve her pain, so she administered a drug that killed Sarah while she slept.

SCENARIO TWO: Tammi and Karen were eating lunch in Tammi's kitchen. Karen made a noise that led Tammi to believe that her friend was choking. Tammi wanted to help Karen, so she forcefully punched her in the back.

SCENARIO THREE: One day, while his neighbors were on vacation, Bob noticed that the shutters on his neighbors' home were dangerously close to falling off their hinges. Because the shutters were located above a busy walkway, he feared that they would fall and hurt someone. Bob used a sledgehammer to destroy and remove the shutters.

SCENARIO FOUR: Janine is a single mother who recently lost her job. She could not afford to buy her young daughter a winter coat. Even though she bundled her daughter in layers of clothing, it was not enough to keep her warm in the frigid weather. Janine was worried about her daughter's health, so she decided to steal a coat from a nearby clothing store.

\section{SURVEY 3 - CONSENT HYPOTHETICALS}

SCENARIO ONE: Janine is a single mother who recently lost her job. She could not afford to buy her young daughter a winter coat. Even though she bundled her daughter in layers of clothing, it was not enough to keep her warm in the frigid weather. Janine was worried about her daughter's health, so she decided to steal a coat from a nearby clothing store. The store-owner watched Janine steal the coat, but he decided not to do anything about it. He knew that Janine was poor and wanted her to have the coat.

SCENARIO TWO: Dr. Smith is a medical doctor. Her patient, Sarah, was dying of cancer. Although Sarah was able to think clearly, she was in a constant state of pain and suffering. Sarah begged Dr. Smith to assist her in committing suicide. Sarah's family supported her decision. Sarah and Dr. Smith live in a place where physician-assisted suicide is legal. Dr. Smith cared about Sarah and wanted to relieve her pain, so she administered a drug that killed Sarah while she slept. 
SCENARIO THREE: One day, while his neighbors were on vacation, Bob noticed that the shutters on his neighbors' home were dangerously close to falling off their hinges. Because the shutters were located above a busy walkway, he feared that they would fall and hurt someone. Bob called his neighbors to explain the situation, and they gave him permission to remove and dispose of the shutters. Bob used a sledgehammer to destroy and remove the shutters.

SCENARIO FOUR: Tammi and Karen were eating lunch in Tammi's kitchen. Karen made a noise that led Tammi to believe that her friend was choking. Karen motioned for Tammi to hit her on the back. Tammi wanted to help Karen, so she forcefully punched her in the back.

\section{SURVEY 4 - OUTCOMES HYPOTHETICALS}

SCENARIO ONE: One day, while his neighbors were on vacation, Bob noticed that the shutters on his neighbors' home were dangerously close to falling off their hinges. Because the shutters were located above a busy walkway, he feared that they would fall and hurt someone. Bob called his neighbors to explain the situation, and they gave him permission to remove and dispose of the shutters. Bob used a sledgehammer to destroy and remove the shutters. Thanks to Bob, no one was hurt.

SCENARIO TWO: Tammi and Karen were eating lunch in Tammi's kitchen. Karen made a noise that led Tammi to believe that her friend was choking. Karen motioned for Tammi to hit her on the back. Tammi wanted to help Karen, so she forcefully punched her in the back. The bit of roast beef sandwich popped out of Karen's mouth and she was able to breathe again. She was grateful that Tammi was there to save her from the choking hazard.

SCENARIO THREE: Janine is a single mother who recently lost her job. She could not afford to buy her young daughter a winter coat. Even though she bundled her daughter in layers of clothing, it was not enough to keep her warm in the frigid weather. Janine worried about her daughter's health, so she decided to steal a coat from a nearby clothing store. The store-owner watched Janine steal the coat, but he decided not to do anything about it. He knew that Janine was poor and wanted her to have the coat. Janine took the coat home to her daughter. Her daughter was able to keep warm and remained healthy in the cold weather.

SCENARIO FOUR: Dr. Smith is a medical doctor. Her patient, Sarah, was dying of cancer. Although Sarah was able to think clearly, she was in a constant state of pain and suffering. Sarah begged Dr. Smith to assist her in committing suicide. Sarah’s family supported her decision. Sarah and Dr. Smith live in a place where physician-assisted suicide is legal. Dr. Smith cared about Sarah and wanted to relieve her pain, so she administered a drug that killed Sarah while she slept. Sarah and her relatives were grateful for Dr. Smith's willingness to honor Sarah's wishes and to put an end her suffering. 


\section{SURVEY 5 - IMMORAL PROTOTYPE HYPOTHETICALS}

SCENARIO ONE: Janine was shopping at a local store and saw a winter coat that she liked. She didn't feel like paying for it, so she decided to steal the coat instead. Later that day, the store-owner noticed that the coat was missing and realized that someone had stolen it. He was very upset about his loss.

SCENARIO TWO: One day, Bob’s neighbors went away on vacation. Bob hates his neighbors and enjoys seeing them upset. In the middle of the night, Bob used a sledgehammer to destroy and remove the shutters on his neighbor's home. After the neighbors returned, they were shocked to see that someone had vandalized their home. All of the shutters were destroyed. They were heartbroken and had to pay a lot of money to replace them.

SCENARIO THREE: Dr. Smith is a medical doctor. Her patient, Sarah, was dying of cancer. Sarah wanted her doctor to help her survive for as long as possible. Dr. Smith hated taking care of Sarah and wished that she would die. In the middle of the night, Dr. Smith administered a drug that killed Sarah in her sleep. Sarah died immediately.

SCENARIO FOUR: Tammi and Karen were eating lunch. Tammi hates Karen and wanted to hurt her. She raised her fist at Karen. Karen didn't want to get hurt, so she tried to move away quickly. Tammi forcefully punched Karen in the back and made her cry. 


\section{STUDY 2}

DIRECTIONS: This questionnaire will ask you to read four different scenarios and to respond to a set of corresponding questions. It is very important to read each scenario carefully and to respond to each question. Your input is greatly appreciated. Thank you for your participation!

QUESTIONS ASKED:

[After each scenario, the participants were asked the same questions, which are as follows:]

1. Think about what [name] did. Were [names's] actions wrong or all right? Write your answer and explain the reason for your decision in the space below.

2. Choose the response that best completes this sentence:

I think that [name's] actions were

very wrong

wrong

somewhat more wrong than right

somewhat more right than wrong

right

very right

3. Should [name] receive any blame or praise for [his/her/their] actions? Choose a response:

full blame

some blame

no blame or praise

some praise

full praise

4. How difficult was it to make a judgment about this scenario?

not difficult at all

somewhat difficult

difficult

very difficult

too difficult to make a judgment 


\section{SURVEY 1 - PROTOTYPE HYPOTHETICALS}

SCENARIO ONE: A group of medical scientists wants to create a market that would allow them to sell cloned human body parts for a profit. The scientists plan to extract tissue from their own bodies to reproduce the organs. The scientists believe that this project will make them wealthy and powerful. They do not care about the people they serve. Recently, they conducted research to see if their patients' bodies would accept the organs. The research was conducted without the patients' knowledge or permission. A number of people died as a result of the experiment.

SCENARIO TWO: Fred decided that it was time to get married. He thought that his neighbor, Anne, would make a good wife. Fred is not in love with Anne and thinks that she is disgusting. However, he wanted a wife to cook and clean for him and to bear his children. As a result, he found a way to force Anne into marrying him. Anne is now miserable and does not want to be married to Fred.

SCENARIO THREE: Sam is the Director of Human Resources for a large factory. Sam cares about people and believes that it is important to help others in need. Recently, Sam met a homeless man who lost both his job and family after a devastating tornado hit his community. In order to help, Sam offered the man a full-time job in the factory. The man was excited about the offer and eagerly accepted it. After several months of training, the man turned out to be an excellent worker and earned a good salary. The man lived a happy, healthy life thanks to Sam.

SCENARIO FOUR: Beth is a devoted advocate for orphaned children. Recently, she created a foundation that helps them find good homes. Voluntary donations are used to take care of the orphans while they are waiting to be adopted. The children and the community value her contribution and are grateful for the assistance it provides. Because of Beth's charity work, the orphans and their new families are living happier, healthier lives.

\section{SURVEY 2 - NONPROTOTYPE: INTENTION CONFLICT HYPOTHETICALS}

SCENARIO ONE: A group of medical scientists wants to create a market that would allow them to sell cloned human body parts for a profit. The scientists plan to extract tissue from their own bodies to reproduce the organs. They care about their patients and believe that increasing the number of vital organs available for transplants will save more lives. In addition, they want to use the profits to help low-income patients pay for organ transplants. Recently, they conducted research to see if their patients' bodies would accept the organs. The research was conducted without the patients' knowledge or permission. A number of people died as a result of the experiment. 
SCENARIO TWO: Anne told Fred that she is lonely and wants to be married. Fred is not in love with Anne and thinks that she is disgusting. However, he cares about Anne as a friend and doesn't like to see her so unhappy. He thought that if he married Anne, she would feel better. Even though Anne told Fred that she wouldn't marry him, he was convinced that she refused his proposal only to be polite. Fred truly believed that Anne would be happy as his wife, so he found a way to force Anne into marrying him. Anne is now miserable and does not want to be married to Fred.

SCENARIO THREE: Sam is a Director of Human Resources for a large factory. Recently, Sam met a homeless man who lost both his job and family after a devastating tornado hit his community. Sam hates the victims of the tornado because he believes that they are needy and self-pitying. Sam wanted to torture this man, so he offered him the most grueling, dangerous job at the factory. The man eagerly accepted it. He liked his job and didn't find it difficult at all. After several months of training, the man turned out to be an excellent worker and earned a good salary. The man lived a happy, healthy life thanks to Sam.

SCENARIO FOUR: Beth recently created a foundation that helps orphaned children find good homes. Voluntary donations are used to take care of the children while they are waiting to be adopted. Beth hates orphaned children and finds them revolting. However, she hopes to run for public office and plans to use her charity work to convince more people to vote for her. The children and the community value her contribution and are grateful for the assistance it provides. Because of Beth's charity work, the orphans and their new families are living happier, healthier lives.

\section{SURVEY 3 - NONPROTOTYPE: CONSENT CONFLICT HYPOTHETICALS}

SCENARIO ONE: A group of medical scientists wants to create a market that would allow them to sell cloned human body parts for a profit. The scientists plan to extract tissue from their own bodies to reproduce the organs. The scientists believe that this project will make them wealthy and powerful. They do not care about the people they serve. Recently, they conducted research to see if the patients' bodies would accept the organs. The scientists tested the organs only on patients who specifically requested them. The patients knew where the organs came from and the risks involved in the procedure before agreeing to participate in the study. A number of people died as a result of the experiment.

SCENARIO TWO: Fred decided that it was time to get married. He thought that his neighbor, Anne, would be a good wife. Fred isn't in love with Anne and thinks that she is disgusting. However, he wanted a wife to cook and clean for him and to bear his children. Anne liked the idea and accepted Fred's offer. Anne is now miserable but wants to remain married to Fred.

SCENARIO THREE: Sam is a Director of Human Resources for a large factory. Sam cares about people and believes that it is important to help others in need. Recently, Sam 
met a homeless man who lost both his job and family after a devastating tornado hit his community. In order to help, Sam offered the man a job in the factory. The man told Sam that he does not want to work at the factory. Instead, he wants to take his time to find a different job. Sam knows that living on the streets is very dangerous, so he found a way to coerce the man into taking the job against his will. After several months of training, the man turned out to be an excellent worker and earned a good salary. The man lived a happy, healthy life thanks to Sam.

SCENARIO FOUR: Beth is a devoted advocate for orphaned children. Recently, she created a foundation that helps them find good homes. Voluntary donations are used to take care of the children while they are waiting to be adopted. Although Beth wants to help the orphans, the children and the community do not want her services. They want to develop their own solutions to the orphan problem. Beth believes that her services are necessary, so she finds a way to force the children and the community to accept her services against their will. Because of Beth's charity work, the orphans and their new families are living happier, healthier lives.

\section{SURVEY 4 - NONPROTOTYPE: SOCIAL CONTEXT CONFLICT HYPOTHETICALS}

SCENARIO ONE: A group of medical scientists wants to create a revolutionary, affordable new drug that has the potential to cure a number of deadly diseases. They believe that this drug will make them wealthy and powerful. They don't care about the people they serve. Recently, they conducted research to see if their patients' bodies will accept the drug. The research was conducted without the patients' knowledge or permission. A number of people died as a result of the experiment.

SCENARIO TWO: Fred decided that it was time to get married. He had been dating his neighbor, Anne, for a long time. Anne and Fred believe that they are soul-mates and love each other deeply. Fred wanted to get married so that Anne would cook and clean for him and bear his children. As a result, Fred found a way to force Anne into marrying him. Anne is now miserable and does not want to be married to Fred.

SCENARIO THREE: Sam is a Director of Human Resources for a large factory. Sam cares about people and believes that it is important to help others in need. Recently, Sam met a homeless boy who lost his family after a devastating tornado hit his community. In order to help, Sam offered the 5-year-old boy a full-time job in the factory. The boy was excited about the offer and eagerly accepted it. The boy lives in a community in which children are allowed to take care of themselves and work full-time. After several months of training, the boy turned out to be an excellent worker and earned a good salary. The boy lived a happy, healthy life thanks to Sam.

SCENARIO FOUR: Beth is a devoted advocate for orphaned children. Recently, she created a baby auction that sells orphans to the highest bidding families. The proceeds are used to take care of orphans while they are waiting to be adopted. The children and the 
community value her contribution and are grateful for the assistance it provides. Because of Beth's charity work, the orphans and their new families are living happier, healthier lives.

\section{SURVEY 5 - NONPROTOTYPE: OUTCOMES CONFLICT HYPOTHETICALS}

SCENARIO ONE: A group of medical scientists wants to create a market that would allow them to sell cloned human body parts for a profit. The scientists plan to extract tissue from their own bodies to reproduce the organs. The scientists believe that this project will make them wealthy and powerful. They do not care about the people they serve. Recently, they conducted research to see if their patients' bodies will accept the organs. The research was conducted without the patients' knowledge or permission. The transplants saved the life of each person who received an organ and increased the number of vital organs available to those who needed them.

SCENARIO TWO: Fred decided that it was time to get married. He thought that his neighbor, Anne, would be a good wife. Fred isn't in love with Anne and thinks that she is disgusting. However, he wanted a wife to cook and clean for him and to bear his children. As a result, he found a way to force Anne into marrying him. After Fred and Anne married, they developed a very strong, blissful relationship. Anne and Fred remained happily married for the rest of their lives.

SCENARIO THREE: Sam is a Director of Human Resources for a large factory. Sam cares about people and believes that it is important to help others in need. Recently, Sam met a homeless man who lost both his job and family after a devastating tornado hit his community. In order to help, Sam offered the man a job in the factory. The man was excited about the offer and eagerly accepted it. After several months of training, the man turned out to be an excellent worker and earned a good salary. However, not long after Sam hired the man, the man died in a factory accident while working.

SCENARIO FOUR: Beth is a devoted advocate for orphaned children. Recently, she created a foundation that helps them find good homes. Voluntary donations are used to take care of the children while they are waiting to be adopted. The children and their new families value her contribution and are grateful for the help that it provides. However, a government study revealed that some children had been psychologically and physically abused after their adoptions were legalized. 


\section{IRB APPROVAL LETTER \\ UNIVERSITY OF ILLINOIS \\ AT CHICAGO}

Office for the Protection of Research Subjects (OPRS)

Office of the Vice Chancellor for Research (MC 672)

203 Administrative Office Building

1737 West Polk Street

Chicago, Illinois 60612-7227

\section{Approval Notice \\ Continuing Review (Response To Modifications)}

March 6, 2012

Carol Larson, Ph.D.

Educational Psychology

\section{RE: $\quad$ Protocol \# 2005-0188 \\ “A Cognitive Prototype Model of Moral Judgment and Disagreement”}

Dear Dr. Larson:

Your Continuing Review (Response To Modifications) was reviewed and approved by the Expedited review process on March 5, 2012. You may now continue your research.

Please note the following information about your approved research protocol:

Protocol Approval Period:

Approved Subject Enrollment \#:

Additional Determinations for Research Involving Minors: These determinations have not been made for this study since it has not been approved for enrollment of minors.

Performance Sites:

Sponsor:

PAF\#:

Research Protocol(s):

a) Features of Moral Dilemmas, as submitted on March 18, 2005

Recruitment Material(s):

a) N/A: Limited to data analysis only

Informed Consent(s):

a) N/A: Limited to data analysis only

Your research meets the criteria for expedited review as defined in 45 CFR 46.110(b)(1) under the following specific category(ies):

(6) Collection of data from voice, video, digital, or image recordings made for research purposes., (7) Research on individual or group characteristics or behavior (including but not limited to research on perception, cognition, motivation, identity, language, communication, cultural beliefs or practices and social behavior) or research employing survey, interview, oral history, focus group, program evaluation, human factors evaluation, or quality assurance methodologies.

Please note the Review History of this submission:

Review Process

Review Date

Review Action 


\begin{tabular}{|l|l|l|l|l|}
\hline $02 / 09 / 2012$ & Continuing Review & Expedited & $02 / 13 / 2012$ & $\begin{array}{l}\text { Modifications } \\
\text { Required }\end{array}$ \\
\hline $02 / 28 / 2012$ & $\begin{array}{l}\text { Response To } \\
\text { Modifications }\end{array}$ & Expedited & $03 / 05 / 2012$ & Approved \\
\hline
\end{tabular}

Please remember to:

$\rightarrow$ Use your research protocol number (2005-0188) on any documents or correspondence with the IRB concerning your research protocol.

$\rightarrow$ Review and comply with all requirements on the enclosure, "UIC Investigator Responsibilities, Protection of Human Research Subjects"

Please note that the UIC IRB has the prerogative and authority to ask further questions, seek additional information, require further modifications, or monitor the conduct of your research and the consent process.

Please be aware that if the scope of work in the grant/project changes, the protocol must be amended and approved by the UIC IRB before the initiation of the change.

We wish you the best as you conduct your research. If you have any questions or need further help, please contact OPRS at (312) 996-1711 or me at (312) 355-0816. Please send any correspondence about this protocol to OPRS at $203 \mathrm{AOB}, \mathrm{M} / \mathrm{C} 672$.

Sincerely,

Alison Santiago, MSW, MJ

IRB Coordinator, IRB \# 2

Office for the Protection of Research

Subjects

Enclosure(s):

1. UIC Investigator Responsibilities, Protection of Human Research Subjects

2. Data Security Enclosure

cc: $\quad$ Keith Thiede, PhD, Educational Psychology, M/C 147

Larry Nucci (Faculty Sponsor), Psychology, M/C 147 


\section{VITA}

NAME:

EDUCATION: Doctor of Philosophy, Educational Psychology

University of Illinois Chicago, IL

Fall 2012

Advanced Certificate, Educational/Organizational Leadership University of Illinois Urbana-Champaign, IL

Fall 2002

Illinois Type 75 certification

Master of Arts, Elementary Education

New York University New York, NY

Summer 1995

Specializing in Multicultural Education and School Anthropology

English Language Learning endorsement

\section{Bachelor of Science, Elementary Education}

Southern Illinois University Carbondale, IL

Winter 1990

Illinois Type 03 certification

Language Arts endorsement

HONORS:

Diversifying Faculty for Illinois Fellow

Mensa Scholarship

University of Illinois at Chicago Provost's Award

La Juventud de Brooklyn Scholarship

PUBLICATIONS: $\quad$ Nucci, L., Drill, K., Larson, C., \& Browne, C. (2005).

Preparing pre-service teachers for character education in urban elementary Schools: The UIC initiative. Journal for Research in Character Education,3(2).

PRESENTATIONS: Why can't we all agree? A cognitive prototype model of moral disagreement. Poster presented at the University of Illinois at Chicago’s Student Research Forum, Spring 2011.

Young Asian-Americans and political representation. Presented at New York University’s Asians in America Conference, Spring 1996. 